\title{
Diastereoselective synthesis
}

\section{3,4-dihydropyran-3-carboxamides with in vitro anti-inflammatory activity}

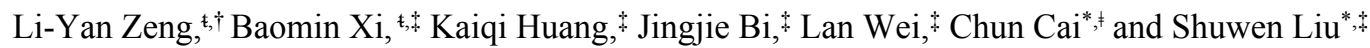 \\ $\dagger$ Guangdong Provincial Key Laboratory of New Drug Screening, School of Pharmaceutical Sciences, \\ Southern Medical University, Guangzhou 510280 China \\ $¥$ Guangdong Provincial Key Laboratory of New Drug Screening, School of Pharmaceutical Sciences, \\ Southern Medical University, Guangzhou 510515, China \\ ${ }^{\ddagger}$ Chemical Engineering College, Nanjing University of Science and Technology, Nanjing 210094, \\ China
}

\section{Content}

Diastereoselective synthesis of 3,4-dihydropyran-3-carboxamides with in vitra

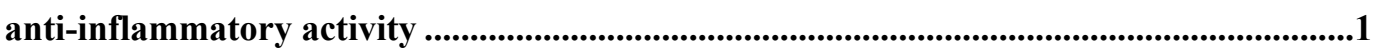

General information: .......................................................................................................................2

The structural identification ...................................................................................................2

Structure of compound $6\{1,1,3,1\}$ from XRD ............................................................................2

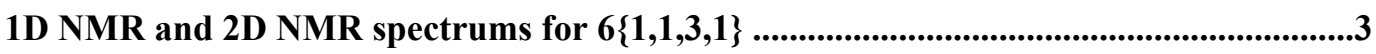

Synthetic procedure:........................................................................................................................8

General method of screening compounds 6 for anti-inflammatory activity via testing

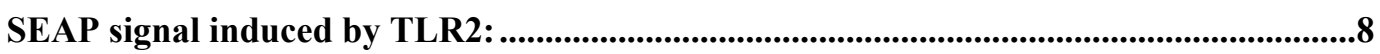

Anti-inflammatory data targeting NF-кB by inhibiting SEAP signal: ................................9

General method for proinflammatory cytokine IL-6 and TNF- $\alpha$ inhibitory activities of

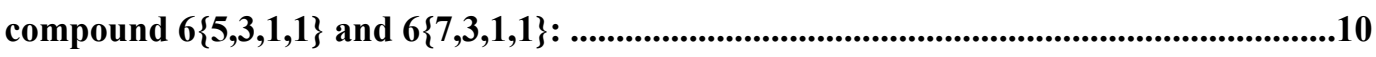

Characterization Data for compounds 6: .....................................................................................11

Copies of NMR Spectra:..................................................................................................................23

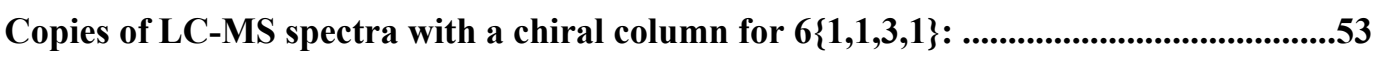




\section{General information:}

All of the reagents were obtained from commercial suppliers and used without further purification. All melting points were uncorrected. All compounds were structurally verified by ${ }^{1} \mathrm{H}$ NMR, ${ }^{13} \mathrm{C}$ NMR and HRMS (or Elemental analyses and LRMs). Compound 6aaca was structural identified by XRD and further confirmed by $1 \mathrm{D}$ NMR and 2D NMR. ${ }^{1} \mathrm{H},{ }^{13} \mathrm{C}$ NMR ${ }^{13} \mathrm{C}$ DEPT $90,{ }^{13} \mathrm{C}$ DEPT 135, COSY, HSQC and HMBC spectra were recorded on a Bruker Advance drx 400 spectrometer operating at $400 \mathrm{MHz}$ or $100 \mathrm{MHz}$, and the chemical shifts are reported in ppm and the coupling constant in Hz. The high-resolution mass (HRMS) spectra were obtained with an LCMS-IT-TOF mass spectrometer, while the LRMS were taken on LC/MS/MS instrument in the electrospray ionization (positive) mode. X-Ray crystallographic analysis was performed with a Bruker APEX2 smart CCD and a Siemens P4 diffractometer. Diastereoselective ratio of compound $\mathbf{6}\{\mathbf{1 , 1 , 3 , 1 \}}$ was confirmed by HPLC-MS with a chiral column. All of the products were new.

\section{The structural identification}

\section{Structure of compound $6\{1,1,3,1\}$ from XRD}

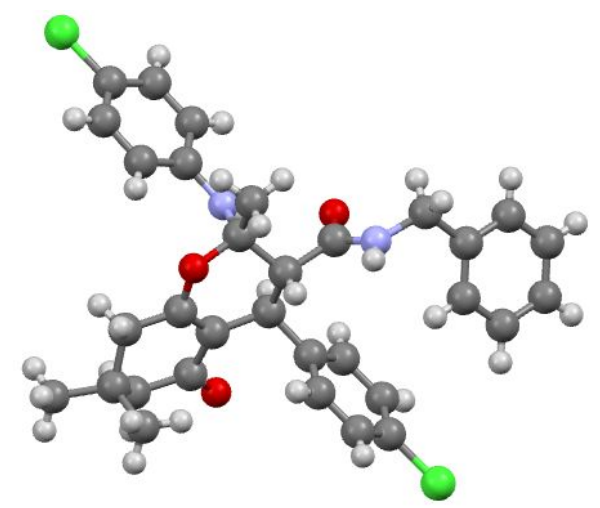

Figure S1. X-ray structure for compound $\mathbf{6}\{1,1,3,1\}$

Crystal data and structure refinement for $6\{1,1,3,1\}$.

Identification code

Empirical formula

Formula weight

Temperature

Wavelength

Crystal system

Space group

Unit cell dimensions

Volume
$6\{1,1,3,1\}$

$\mathrm{C} 32 \mathrm{H} 32 \mathrm{Cl} 2 \mathrm{~N} 2 \mathrm{O} 3$

563.49

296(2) K

$0.71073 \AA$

Monoclinic

$\mathrm{P} 2 / \mathrm{n}$

$\mathrm{a}=10.2178(12) \AA \quad \alpha=90^{\circ}$.

$\mathrm{b}=10.5633(12) \AA \quad \beta=96.495(4)^{\circ}$.

$\mathrm{c}=31.638(4) \AA \quad \gamma=90^{\circ}$.

$3392.9(7) \AA^{3}$ 
Z

Density (calculated)

Absorption coefficient

$\mathrm{F}(000)$

Crystal size

Theta range for data collection

Index ranges

Reflections collected

Independent reflections

Completeness to theta $=25.190^{\circ}$

Absorption correction

Refinement method

Data / restraints / parameters

Goodness-of-fit on $\mathrm{F}^{2}$

Final $\mathrm{R}$ indices [I $>2 \operatorname{sigma}(\mathrm{I})]$

$\mathrm{R}$ indices (all data)

Extinction coefficient

Largest diff. peak and hole
4

$1.103 \mathrm{Mg} / \mathrm{m}^{3}$

$0.222 \mathrm{~mm}^{-1}$

1184

$0.220 \times 0.200 \times 0.180 \mathrm{~mm}^{3}$

1.296 to $25.190^{\circ}$.

$-10<=\mathrm{h}<=12,-12<=\mathrm{k}<=12,-36<=\mathrm{l}<=37$

21777

$6106[\mathrm{R}(\mathrm{int})=0.0798]$

$99.9 \%$

Semi-empirical from equivalents

Full-matrix least-squares on $\mathrm{F}^{2}$

$6106 / 0 / 355$

0.908

$\mathrm{R} 1=0.0615, \mathrm{wR} 2=0.1587$

$\mathrm{R} 1=0.1306, \mathrm{wR} 2=0.1981$

$\mathrm{n} / \mathrm{a}$

0.249 and -0.284 e. $\AA^{-3}$

1D NMR and 2D NMR spectrums for $6\{1,1,3,1\}$

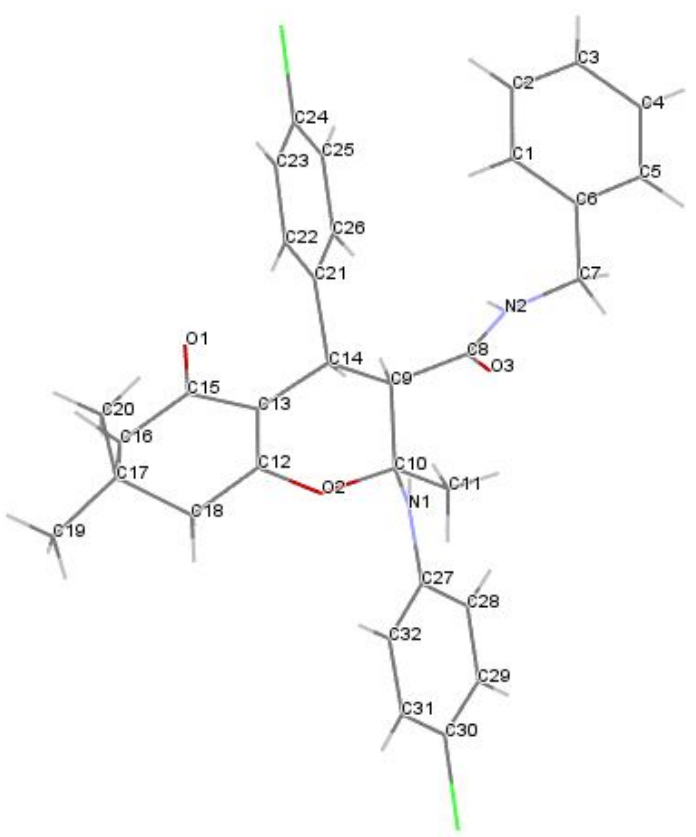

Figure S2. Structure of compound $\mathbf{6}\{\mathbf{1 , 1 , 3 , 1}\}$ 

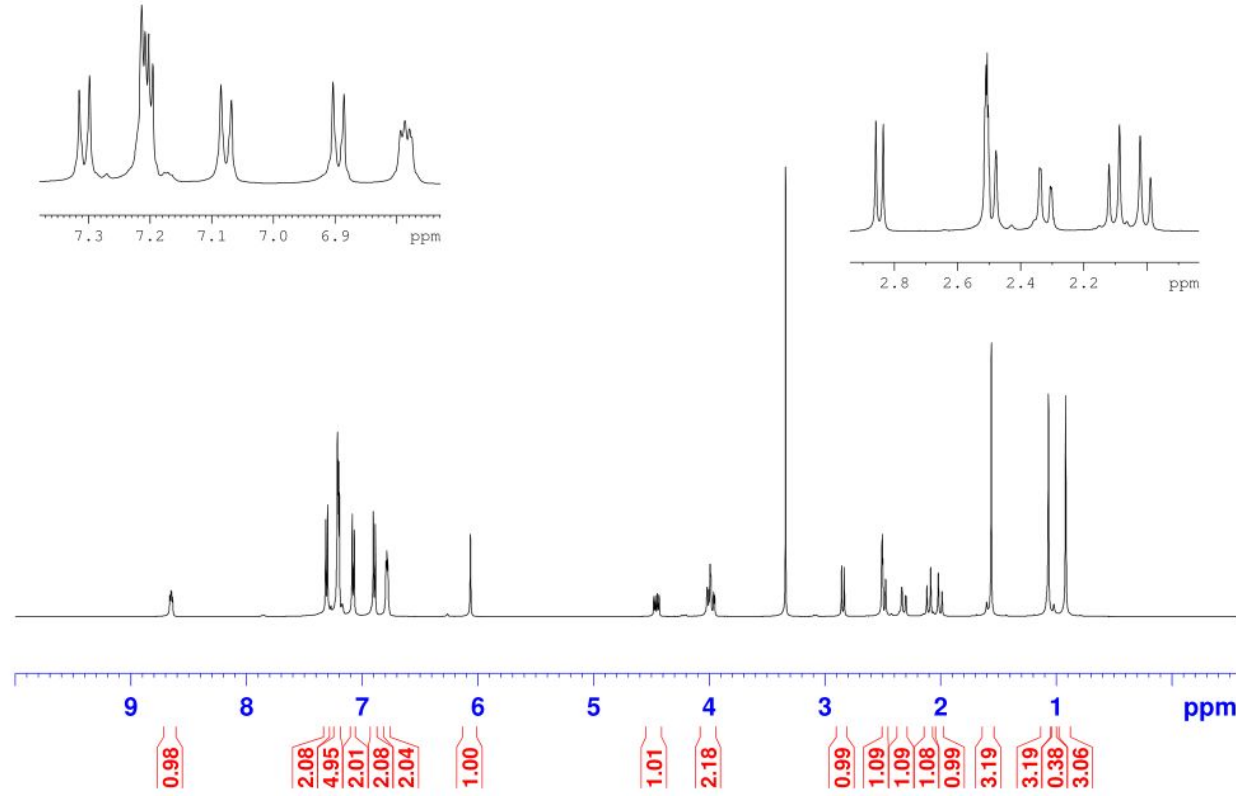

Figure S3. ${ }^{1} \mathrm{H}$ NMR spectrum of compound $6\{1,1,3,1\}$

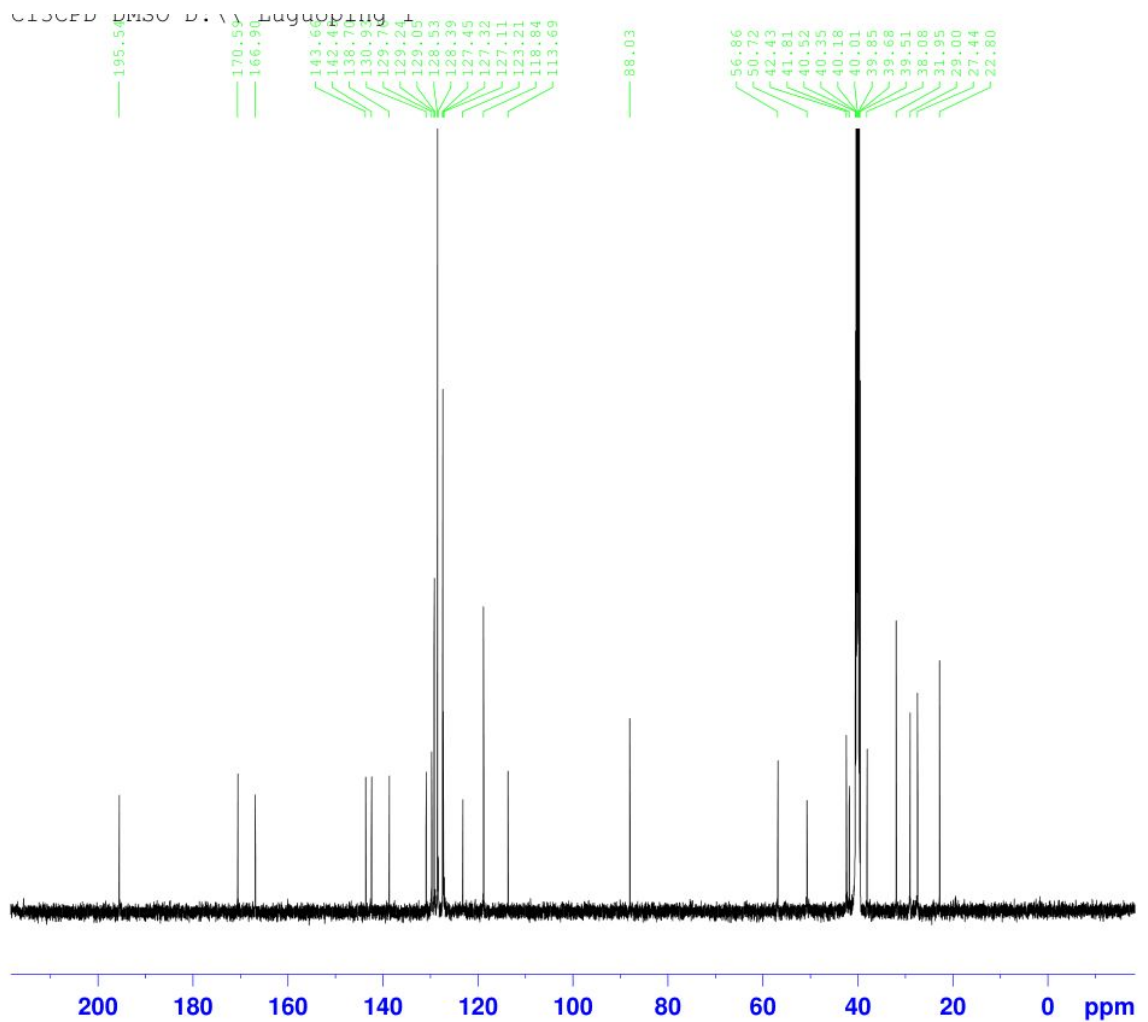

Figure S4. ${ }^{13} \mathrm{C}$ NMR spectrum of compound $6\{1,1,3,1\}$. 


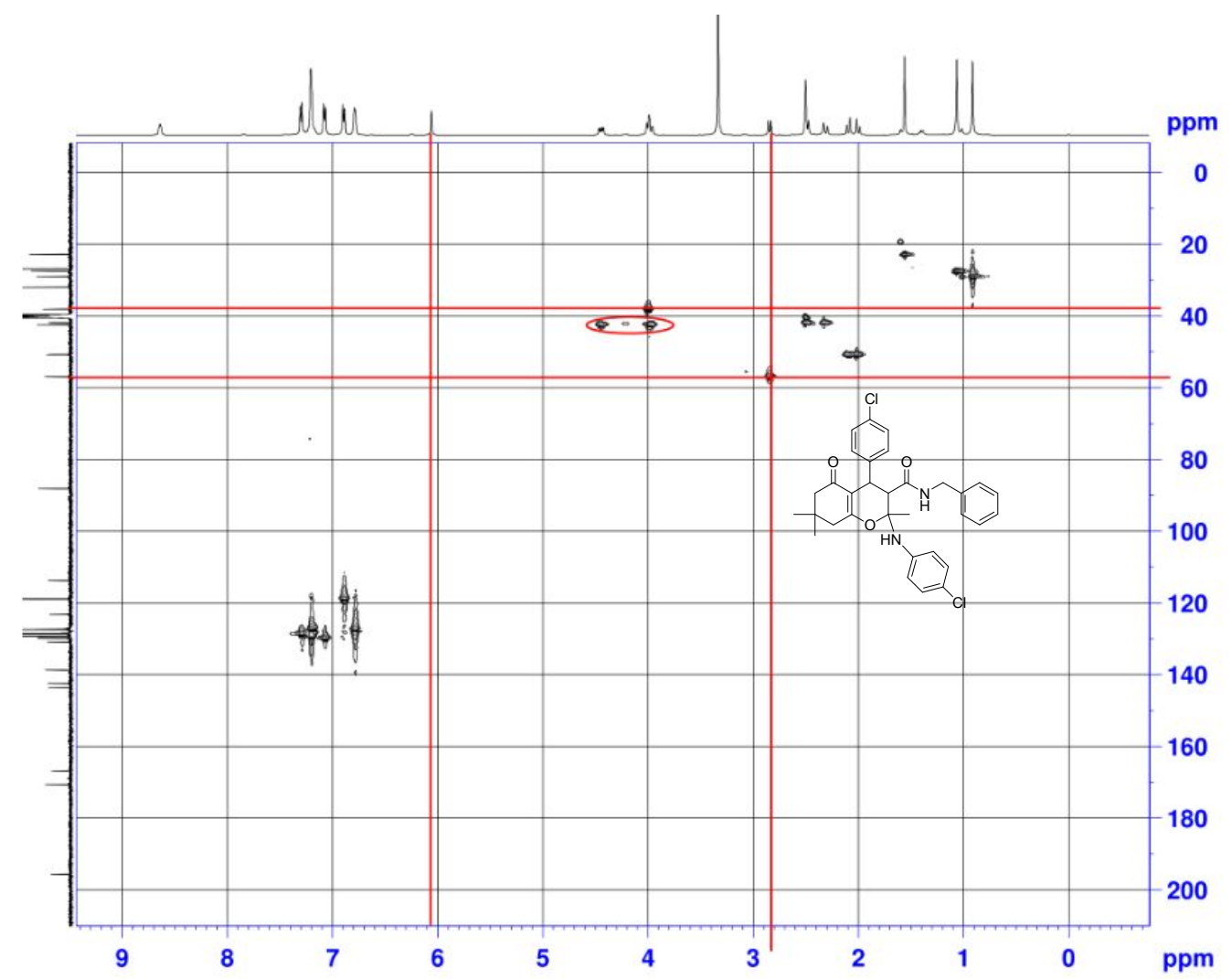

Figure S5. HSQC Spectrum for the Product $\mathbf{6}\{1,1,3,1\}$.

In the HSQC spectrum (Figure S5), no carbon signal is correlated to the proton singlet signal at $\delta=$ $6.06 \mathrm{ppm}$, and multiple proton signal at $\delta=3.96-4.02 \mathrm{ppm}$ is correlated to two carbon signals $\left(\delta_{\mathrm{C}}\right.$ $38.0842 .43)$, one of which $\left(\delta_{\mathrm{C}} 38.08\right)$ is correlated to proton multiple signal at $\delta=3.99-4.02 \mathrm{ppm}$, while the correlation between another carbon signal $\left(\delta_{\mathrm{C}} 42.43\right)$ and two proton signals $\left(\delta_{\mathrm{H}}\right.$ 4.43-4.48 and 3.92-3.97) is observed. All of the observations draw conclusions that, in ${ }^{1} \mathrm{H}$ NMR, the singlet signal at $\delta=6.06 \mathrm{ppm}$ is assignable to the $\mathrm{Ph}-\mathrm{NH}(\mathrm{N} 1)$, while the $\mathrm{CH}_{2}(\mathrm{C} 7)$ adjacent to nitrogen in amide resonated as two multiple signals at $\delta_{\mathrm{H}} 4.43-4.48$ and 3.92-3.97 ppm. Thereby, the explanation for the proton signals at $\delta=3.99-4.02$ and $2.84-2.86 \mathrm{ppm}$ was in need.

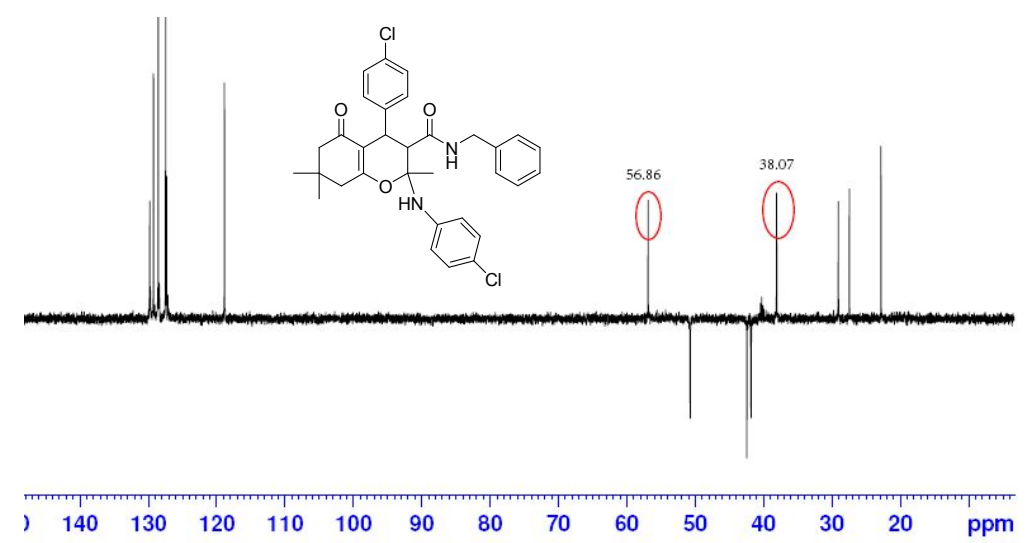

Figure S6. ${ }^{13} \mathrm{C}$ DEPT 135 Spectrum for the Product $\mathbf{6}\{\mathbf{1 , 1 , 3 , 1}\}$ 
According to the HSQC, the correlated carbon signals to the proton signals at $\delta=3.99-4.02$ for ${ }^{1} \mathrm{H}$ and 2.84-2.86 for ${ }^{1} \mathrm{H}$ are $\delta_{\mathrm{C}} 38.08$ and $56.86 \mathrm{ppm}$ respectively, both of which showed positive signals in DEPT135 spectrum (Figure S6).
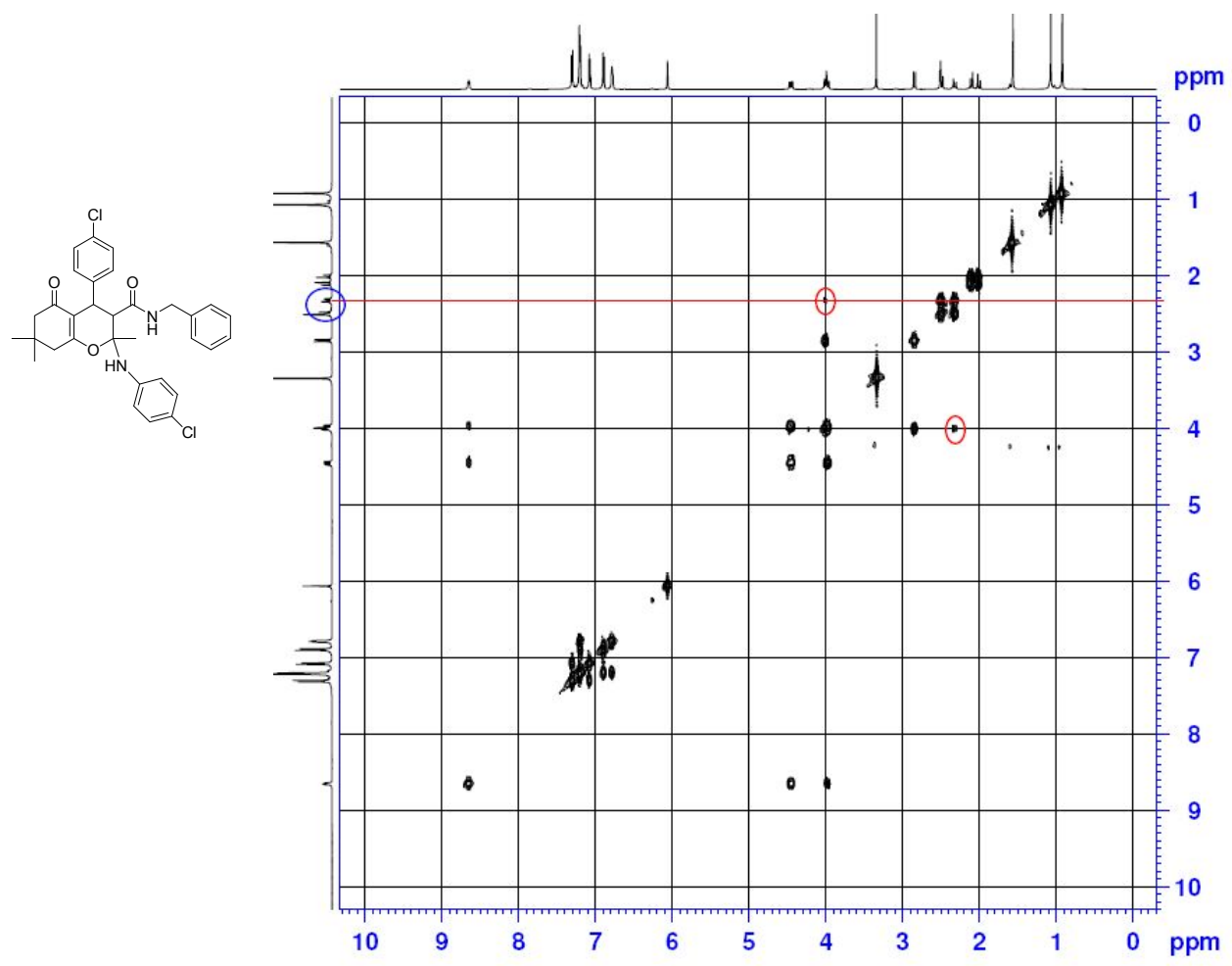

Figure S7. COSY Spectrum for the Product $6\{1,1,3,1\}$.

The COSY spectrum (Figure S7) helped to locate the precise position of the two $\mathrm{CH}$. As been marked in Figure 4 with blue circle, the proton signals at $\delta=3.99-4.02$ is correlated to the $\mathrm{CH}_{2}$ introduced from 5,5-dimethylcyclohexane-1,3-dione 3 which was also marked with blue circle in structure. This phenomenon implied that one of the two $\mathrm{CH}$ in piperding-ring was adjacent to the $\mathrm{CH}_{2}$ marked with blue circle. So the proton signals at $\delta=3.99-4.02$ and $2.84-2.86 \mathrm{ppm}$ is recognized as arising from two $\mathrm{CH}$ in piperdine-ring (No. $13 \mathrm{CH}$ and No. $12 \mathrm{CH}$ in respective). These results further supported the structure of product $\mathbf{6}\{\mathbf{1}, \mathbf{1 , 3}, \mathbf{1}\}$ 2-(4-chlorophenylamino)- $N$-benzyl-4-(4-chlorophenyl)-3,4,5,6,7,8-hexahydro-2,7,7-trimethyl-5-o xo-2H-chromene-3-carboxamide (Figure S3, $\mathbf{6}\{\mathbf{1 , 1 , 3 , 1}\}$ ).

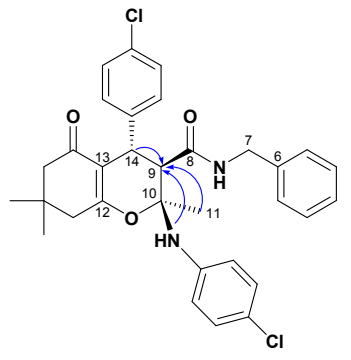

$6\{1,1,3,1\}$ 


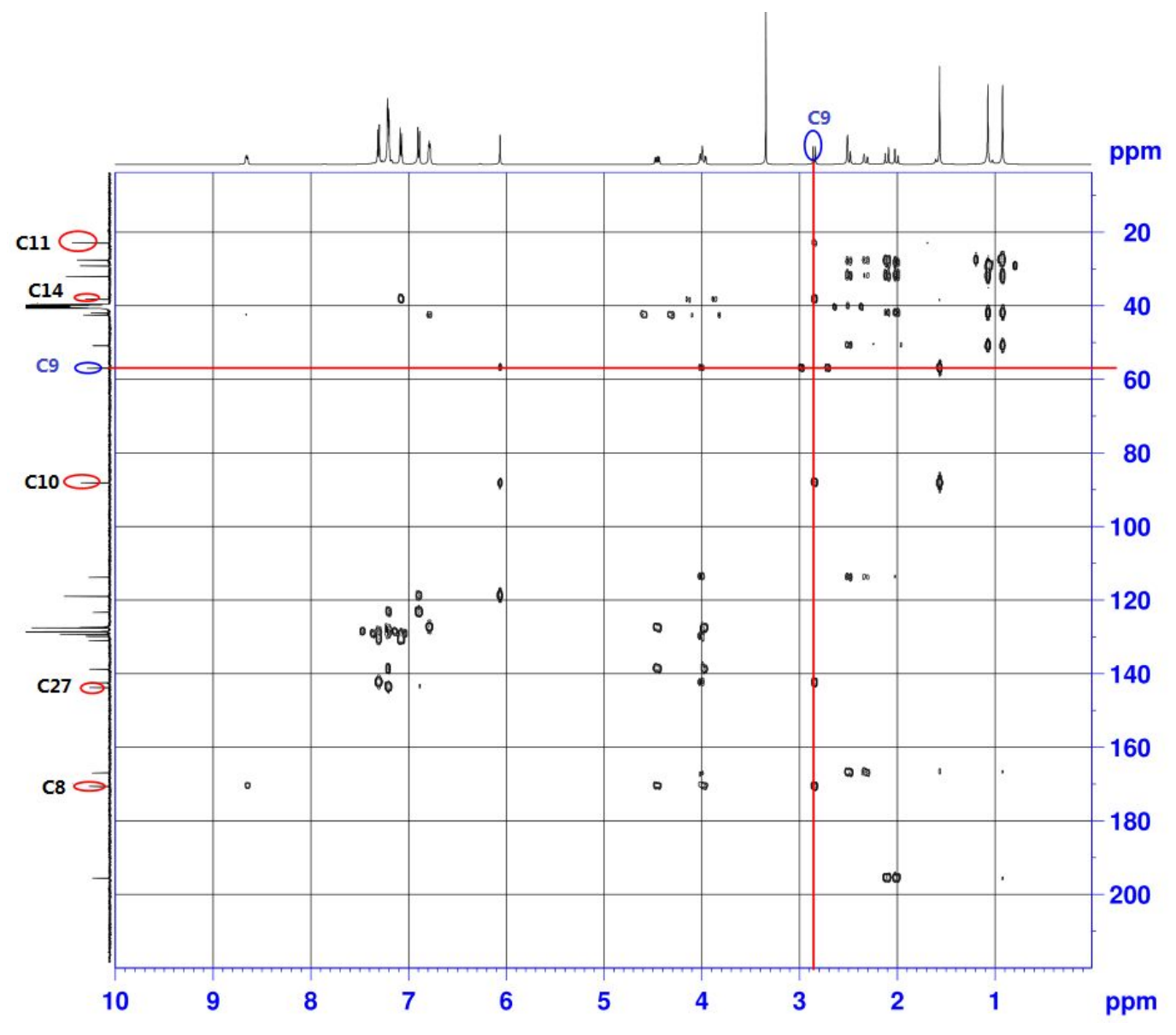

Figure S8. HMBC Spectrum for the Product $6\{1,1,3,1\}$.

The HMBC spectrum gave another evidence for the structure $\mathbf{6}\{\mathbf{1 , 1 , 3 , 1}\}$. (Figure S8) The proton signal at 2.84-2.86 ppm arising from $\mathrm{CH}$ (C9), marked with blue circle in HMBC spectrum, is correlated with carbon signals at $\delta_{\mathrm{C}}=22.80,38.08,88.03,143.66$ and $170.59 \mathrm{ppm}$ arising from $\mathrm{C} 11, \mathrm{C} 14, \mathrm{C} 10, \mathrm{C} 27$ and $\mathrm{C} 8$ respectively. Moreover, the carbon signal at $\delta_{\mathrm{C}}=55.86$ for C9 is also correlated with the proton signals for the $\mathrm{Ph}-\mathrm{NH}, \mathrm{CH}_{3}$ (No. 11) and $\mathrm{CH}$ (No. 14), the correlations was marked with blue arrow in structure $\mathbf{6}\{1,1,3,1\}$ as following. 


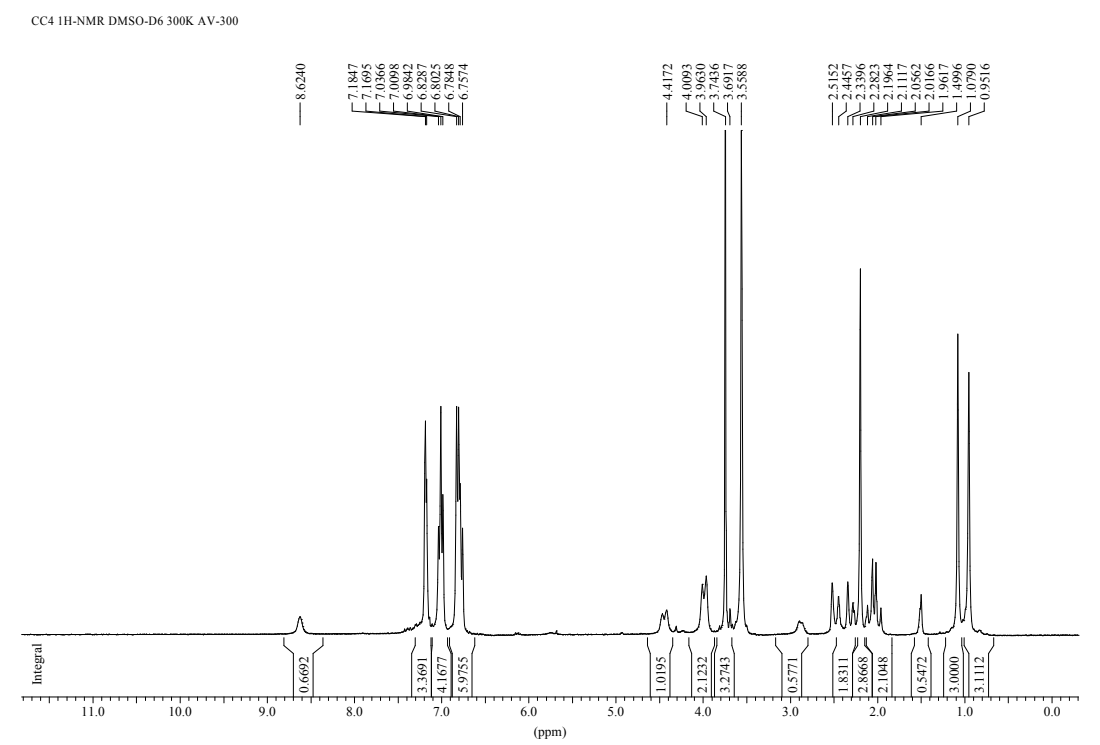

Figure S9. ${ }^{1} \mathrm{H}$ NMR of $\mathrm{D}_{2} \mathrm{O}$ Exchange Spectrum for the Product $\mathbf{6}\{\mathbf{1 , 1 , 1 , 4}\}$

The two N-H proton signals were further confirmed by $\mathrm{D}_{2} \mathrm{O}$ exchange experiment (Figure S9) toward the product $6\{1,1,1,4\}$ (Table 2, Entry 4), with reduced ratio of the N-H proton at $\delta=8.72$ ppm and the disappearance of the signal at $\delta=5.75 \mathrm{ppm}$.

\section{Synthetic procedure:}

The typical procedure for the synthesis of (2S,3R,4S)-2-(p-tolylamino)- $N$-benzyl-4-(4-chlorophenyl)-3,4,5,6,7,8-hexahydro-2,7,7-trimeth yl-5-0xo-2 $H$-chromene-3-carboxamide $6\{1,1,1,1\}$ :

After a solution of benzyl amine $\mathbf{2}\{\mathbf{1}\}(2 \mathrm{mmol}, 0.214 \mathrm{~g})$ and diketene $\mathbf{1}(2 \mathrm{mmol}, 0.168 \mathrm{~g})$ was stirred in $5 \mathrm{ml}$ of MeCN at room temperature for 2 hours, 5,5-dimethylcyclohexane-1,3-dione 3\{1 $\}$ (2 mmol, 0.28g), $p$-toluidine 4\{1\} (2 mmol, 0.214g) and 4-chlorobenzaldehyde 5\{1\} (2 mmol, $0.28 \mathrm{~g})$ were added simultaneously, and then iodine $(0.2 \mathrm{mmol}, 0.051 \mathrm{~g})$ was added to reaction mixture, 8 minutes later, a mass of precipitates were observed, and the reaction mixture was solidified in 20 minutes. $2 \mathrm{ml}$ of $\mathrm{MeCN}$ was added, the reaction mixture was filtrated and washed with a solution of sodium thiosulfate followed by water. The white product $N$-benzyl-4-(4-chlorophenyl)-2-hydroxy-2,7,7-trimethyl-5-oxo-1- $p$-tolyl-1,2,3,5,6,7,8,8a-octahydr oquinoline-3-carboxamide $\mathbf{6}\{\mathbf{1}, \mathbf{1}, \mathbf{1}, \mathbf{1}\}$ was obtained after drying in $95 \%(1.04 \mathrm{~g})$.

General method of screening compounds 6 for anti-inflammatory activity via testing SEAP signal induced by TLR2:

QUANTI-Blue SEAP assay:

HEK-Blue hTLR2 cells were cultured in DMEM supplemented with $10 \%$ FBS, 10× penicillin/streptomycin. Cells were grew in 96-well plates $\left(4 \times 10^{4}\right.$ cells per well) with a volume 
$200 \mu 1$ growth medium (DMEM supplemented with $10 \%$ FBS, $1 \%$ penicillin/streptomycin) per well at $37^{\circ} \mathrm{C}$ constant temperature incubator. The next day, medium was removed from the 96-well plates and replaced with fresh DMEM. After transferring a certain concentration of $100 \mu 1$ drug solution, $50 \mu 1$ DMEM containing (final conc. $10 \mathrm{ng} / \mathrm{ml}$ ) and DMEM were added to $200 \mu \mathrm{l}$ totally, incubated for 24 hours at $37^{\circ} \mathrm{C}$ and set blank and positive control. On the third day, $50 \mu \mathrm{l}$ supernatant were transferred into another 96-well plates and each well was treated with $50 \mu \mathrm{l}$ of QUANTI-Blue (InvivoGen) buffer. Waiting for 15 minutes, a purple color can be observed and then tested the result at an absorbance of $620 \mathrm{~nm}$ with a plate reader.

\section{MTT cell viability assay:}

On the first day, 40,000 HEK-Blue hTLR2 cells per well were implanted to a 96-well plates in $200 \mu$ l of growth medium (DMEM supplemented with $10 \%$ FBS, $1 \%$ penicillin/streptomycin). The plates were incubated at $37^{\circ} \mathrm{C}, 5 \% \mathrm{CO}_{2}$ for 24 hours. The next day, medium was removed from the 96-well plate and replaced with $200 \mu$ fresh DMEM, including indicated concentrations of compound and $\mathrm{Pam}_{3} \mathrm{CSK}_{4}$ (final conc. $10 \mathrm{ng} / \mathrm{ml}$ ), and then was incubated at $37^{\circ} \mathrm{C}$ for 24 hours. After 24 hours of treatment, $15 \mu \mathrm{l}$ MTT solution $(5 \mathrm{mg} / \mathrm{mL}$ in PBS) was added to each well, and then was placed in $37^{\circ} \mathrm{C}$ constant temperature incubator for 3 hours. After that, the liquid of each

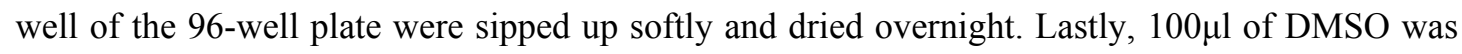
added to each well and then put it on the shaker for 30 minutes (sealed with foil), and the plate was measured at an absorbance of $570 \mathrm{~nm}$ through a plate reader.

\section{Anti-inflammatory data targeting NF-кB by inhibiting SEAP signal:}

Table S1. SEAP signaling inhibition of 16 compounds in HEK-blue hTLR2 cell.

\begin{tabular}{|c|c|c|c|c|c|}
\hline \multirow{3}{*}{ Compound } & \multicolumn{2}{|c|}{$\begin{array}{c}\text { Inhibition percentage } \\
(\%)^{[\dagger]}\end{array}$} & \multirow{3}{*}{ Compound } & \multirow{2}{*}{\multicolumn{2}{|c|}{$\begin{array}{c}\text { Inhibition percentage } \\
(\%)\end{array}$}} \\
\hline & & & & & \\
\hline & $50 \mu \mathrm{M}$ & $100 \mu \mathrm{M}$ & & $50 \mu \mathrm{M}$ & $100 \mu \mathrm{M}$ \\
\hline $6\{1,1,3,1\}$ & $-[*]$ & -[*] & $6\{1,3,3,1\}$ & - & - \\
\hline $6\{1,2,1,1\}$ & - & - & $6\{5,3,1,1\}$ & 63.05 & 97.23 \\
\hline $6\{1,1,1,6\}$ & - & - & $6\{7,3,1,1\}$ & 44.84 & 91.27 \\
\hline $6\{1,1,1,7\}$ & - & - & $6\{1,4,1,1\}$ & - & - \\
\hline $6\{1,2,1,2\}$ & - & - & $6\{1,2,3,1\}$ & - & - \\
\hline $6\{1,3,1,2\}$ & - & - & $6\{1,4,1,4\}$ & - & - \\
\hline $6\{1,3,1,1\}$ & - & - & $6\{1,4,3,1\}$ & - & - \\
\hline $6\{1,3,1,4\}$ & - & - & $6\{1,4,1,2\}$ & - & - \\
\hline
\end{tabular}

$[\dagger]$ The values of compounds were determined from three independent repeats. [\$]No inhibitory activity was detected at the tested concentrations. 
(A)

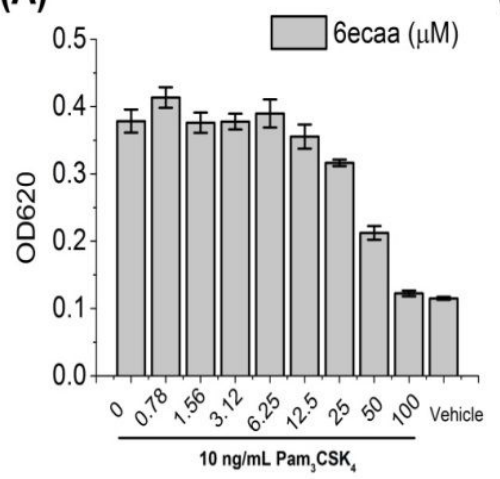

(B)

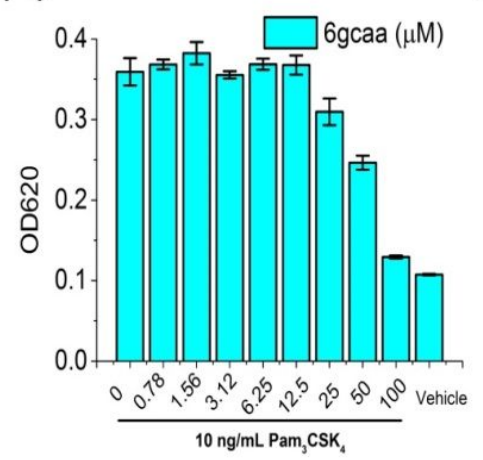

(C)

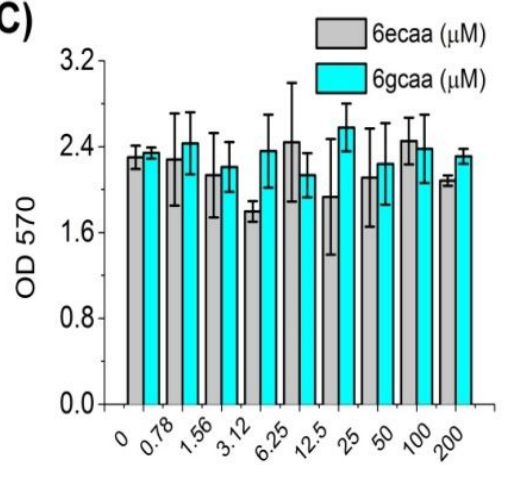

Figure S10. Inhibitory activity and viability of $6\{5,3,1,1\}$ and $6\{7,3,1,1\}$ in HEK-Blue hTLR2 cells. (A) With $10 \mathrm{ng} / \mathrm{ml} \mathrm{Pam}_{3} \mathrm{CSK}_{4}$ as positive control, 6ecaa inhibit SEAP signaling in a dose-dependent manner. Compound $\mathbf{6}\{\mathbf{5 , 3 , 1 , 1}\}$ starts to suppress at a concentration of $6.25 \mu \mathrm{M}$, reaching a maximum level at a concentration of $100 \mu \mathrm{M}$ with $\mathrm{IC}_{50}$ at $41.68 \pm 1.05 \mu \mathrm{M}$. (B) With $10 \mathrm{ng} / \mathrm{ml} \mathrm{Pam}{ }_{3} \mathrm{CSK}_{4}$ as positive control, compound $\mathbf{6}\{\mathbf{7 , 3 , 1 , 1}\}$ inhibit SEAP signaling in a dose-dependent manner with $\mathrm{IC}_{50}$ at $52.86 \pm 2.37 \mu \mathrm{M}$. (C) The viability assay indicated that both 6ecaa and 6gcaa have no toxicity up to $200 \mu \mathrm{M}$. Data are shown as mean \pm s.d. of triplicates.

Table S2. Highlighted results by screening compounds $(2 S, 3 R, 4 S)$-chromene-3-carboxamides 6 for the SEAP signal inhibition. ${ }^{a}$

\begin{tabular}{|c|c|c|}
\hline Commnoind & Ir... (, & $r$ r... \\
\hline $6\{5311\}$ & 41 68+1 ก. & $>2 n \cap$ \\
\hline 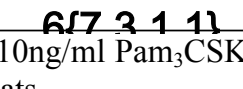 & $\begin{array}{l}5286+227 \\
\text { itive control. }{ }^{b} \text { The }\end{array}$ & $\frac{2 n n}{\text { from three ir }}$ \\
\hline
\end{tabular}

General method for proinflammatory cytokine IL-6 and TNF- $\alpha$ inhibitory activities of compound $6\{5,3,1,1\}$ and $6\{7,3,1,1\}$ :

Cell culture experiment

RAW264.7 cells were used in the current study. Cells were cultured in DMEM medium supplemented with $10 \%$ FBS and $1 \%$ Penicillin-Streptomycin, and incubated at $37^{\circ} \mathrm{C}$ with $5 \%$ $\mathrm{CO}_{2}$. The compound was dissolved in DMSO and co-treated with LPS $(100 \mathrm{ng} / \mathrm{ml})$. After $6 \mathrm{~h}$ incubation, total RNA was isolated using TRIzol reagent and the reverse transcript reaction was carried out with a reverse transcript enzyme (TOYOBO). TNF- $\alpha$ and IL-6 mRNA levels were monitored by Real-time PCR using primers as follows: TNF- $\alpha$-left: CCACCACGCTCTTCTGTCTAC; TNF- $\alpha$-right: AGGGTCTGGGCCATAGAACT; IL-6-left: TGATGCACTTGCAGAAAACA; IL-6-right: ACCAGAGGAAATTTTCAATAGGC. 


\section{Characterization Data for compounds 6:}

(2S,3R,4S)-2-(p-tolylamino)- $N$-benzyl-4-(4-chlorophenyl)-3,4,5,6,7,8-hexahydro-2,7,7-trimeth yl-5-oxo-2H-chromene-3-carboxamide $6\{1,1,1,1\}$ :

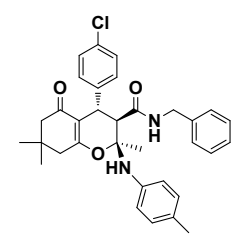

White powder (yield 95\%); m.p. $166-168^{\circ} \mathrm{C} ;{ }^{1} \mathrm{H}$ NMR (500MHz, DMSO- $\left.d 6\right) \delta$ 8.62-8.65 (m, NH, 1H), $7.29(\mathrm{~d}, J=14.0 \mathrm{~Hz}$, aromatic, $2 \mathrm{H}), 7.17-7.19(\mathrm{~m}$, aromatic, 3H), $7.06(\mathrm{~d}$, $J=14.0 \mathrm{~Hz}$, aromatic, $2 \mathrm{H}), 6.97(\mathrm{~d}, J=13.5 \mathrm{~Hz}$, aromatic, $2 \mathrm{H}), 6.73-6.76$ (m, aromatic, $4 \mathrm{H}), 5.75$ (s, NH, 1H), 4.41-4.48 (m, CH, 1H), 3.92-3.99 (m, $\left.\mathrm{CH}_{2}, 2 \mathrm{H}\right), 2.81$ (d, $\left.J=19.0 \mathrm{~Hz}, \mathrm{CH}, 1 \mathrm{H}\right)$, 2.29-2.48 (m, $\left.\mathrm{CH}_{2}, 2 \mathrm{H}\right), 2.17\left(\mathrm{~s}, \mathrm{CH}_{3}, 3 \mathrm{H}\right), 1.95-2.13\left(\mathrm{~m}, \mathrm{CH}_{2}, 2 \mathrm{H}\right), 1.50\left(\mathrm{~s}, \mathrm{CH}_{3}, 3 \mathrm{H}\right), 1.06(\mathrm{~s}$, $\left.\mathrm{CH}_{3}, 3 \mathrm{H}\right), 0.93$ (s, $\left.\mathrm{CH}_{3}, 3 \mathrm{H}\right) ;{ }^{13} \mathrm{C}$ NMR (125MHz, DMSO- $\left.d 6\right) \delta$ 170.8, 167.2, 160.1, 142.7, 142.3, $138.8,130.9,130.0,129.8,128.6,127.5,127.3,122.1,117.9,114.9,113.7,88.5,57.0,50.8,42.4$, 42.0, 38.2, 31.9, 29.3, 27.4, 23.0, 20.6; $\mathrm{MS}\left(\mathrm{ESI}^{+}\right) \mathrm{m} / \mathrm{z} 543(\mathrm{M}+\mathrm{H})$; Anal. Calcd for $\mathrm{C}_{33} \mathrm{H}_{35} \mathrm{ClN}_{2} \mathrm{O}_{3}$ : C, 72.98; H, 6.50; N, 5.16. Found: C, 72.91; H, 6.59; N, 5.02.

$(2 S, 3 R, 4 S)$-2-(p-tolylamino)- $N$-benzyl-3,4,5,6,7,8-hexahydro-2,7,7-trimethyl-5-oxo-4-phenyl-2 $H$-chromene-3-carboxamide $6\{1,1,1,2\}$ :

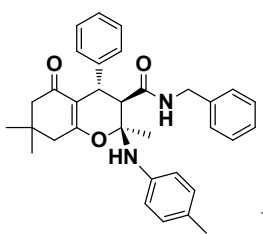

White powder (yield 74\%); m.p. $178-180^{\circ} \mathrm{C} ;{ }^{1} \mathrm{H}$ NMR (500 MHz, DMSO) $\delta 8.69$ (s, NH,1H), 7.31 (d, $J=7.4 \mathrm{~Hz}$, aromatic, 2H), 7.20 (s, aromatic,4H), $7.14-7.03$ (m, aromatic, $5 \mathrm{H}), 6.81$ (s, aromatic, 2H), $6.69(\mathrm{~s}$, aromatic, $1 \mathrm{H}), 5.88(\mathrm{~s}, \mathrm{CH}, 1 \mathrm{H}), 4.43(\mathrm{dd}, J=14.6,6.2 \mathrm{~Hz}$, $\mathrm{CH}, 1 \mathrm{H}), 4.03$ (t, $J=13.4 \mathrm{~Hz}, 2 \mathrm{CH}), 2.87$ (d, $J=10.9 \mathrm{~Hz}, \mathrm{CH}, 1 \mathrm{H}), 2.49-2.28$ (m, $\left.\mathrm{CH}_{2}, 2 \mathrm{H}\right)$, 2.15 (s, $\left.\mathrm{CH}_{2}, 1 \mathrm{H}\right), 2.12\left(\mathrm{~s}, \mathrm{CH}_{3}, 3 \mathrm{H}\right), 2.00\left(\mathrm{~d}, J=16.2 \mathrm{~Hz}, \mathrm{CH}_{2}, 1 \mathrm{H}\right), 1.56\left(\mathrm{~s}, \mathrm{CH}_{3}, 3 \mathrm{H}\right), 1.05$ (d, $J$ $=27.3 \mathrm{~Hz}, 3 \mathrm{H}), 0.93(\mathrm{~s}, 3 \mathrm{H}) ;{ }^{13} \mathrm{C}$ NMR $(125 \mathrm{MHz}, \mathrm{DMSO}) \delta$ 195.56, 170.95, 167.07, 142.98, $142.59,138.73,130.83,129.78,128.51,127.49,127.29,127.03$, 124.87, 119.38, 116.47, 113.68, 88.44, 57.16, 50.66, 42.48, 41.87, 38.31, 31.85, 29.22, 27.31, 23.18, 17.96. MS (ESI $\left.{ }^{+}\right) \mathrm{m} / z$ 509(M $+\mathrm{H}$ ); Anal. Calcd for $\mathrm{C}_{33} \mathrm{H}_{36} \mathrm{~N}_{2} \mathrm{O}_{3}$ : C, 77.92; H, 7.13; N, 5.51. Found: C, 77.06; H, 7.17; N, 5.60. (2S,3R,4S)-2-( $p$-tolylamino)- $N$-benzyl-4-(4-bromophenyl)-3,4,5,6,7,8-hexahydro-2,7,7-trimet hyl-5-oxo-2H-chromene-3-carboxamide $6\{1,1,1,3\}$ :

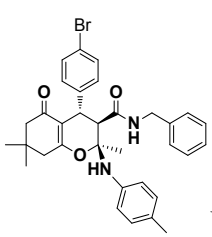

White powder (yield $86 \%$ ); m.p. $183-184^{\circ} \mathrm{C} ;{ }^{1} \mathrm{H}$ NMR (300MHz, DMSO- $d 6$ ) $\delta 8.65$ $(\mathrm{m}, \mathrm{NH}, 1 \mathrm{H}), 7.42-7.44(\mathrm{~m}$, aromatic, $2 \mathrm{H}), 7.27-7.28(\mathrm{~m}$, aromatic, $3 \mathrm{H})$, 6.97-7.03 $(\mathrm{m}$, aromatic, $4 \mathrm{H}), 6.76(\mathrm{~d}, J=3.0 \mathrm{~Hz}$, aromatic, $4 \mathrm{H}), 5.75(\mathrm{~s}, \mathrm{NH}, 1 \mathrm{H}), 4.42-4.50\left(\mathrm{~m}, \mathrm{CH}_{2}, 1 \mathrm{H}\right), 3.96(\mathrm{~d}, J=$ 
$\left.12.0 \mathrm{~Hz}, \mathrm{CH}+\mathrm{CH}_{2}, 2 \mathrm{H}\right), 2.82(\mathrm{~d}, J=11.0 \mathrm{~Hz}, \mathrm{CH}, 1 \mathrm{H}), 2.30-2.46\left(\mathrm{~m}, \mathrm{CH}_{2}, 2 \mathrm{H}\right), 2.18\left(\mathrm{~s}, \mathrm{CH}_{3}\right.$, $3 \mathrm{H}), 1.96-2.14\left(\mathrm{~m}, \mathrm{CH}_{2}, 2 \mathrm{H}\right), 1.51\left(\mathrm{~s}, \mathrm{CH}_{3}, 3 \mathrm{H}\right), 1.07$ (s, $\left.\mathrm{CH}_{3}, 3 \mathrm{H}\right), 0.95\left(\mathrm{~s}, \mathrm{CH}_{3}, 3 \mathrm{H}\right) ;{ }^{13} \mathrm{C} \mathrm{NMR}$ $\left(101 \mathrm{MHz}, \mathrm{CDCl}_{3}\right) \delta 195.98,170.70,167.28,142.21,141.72,136.83,131.67,129.53,129.04$, $128.69,127.66,127.50,120.09,117.73,113.61,88.38,59.35,50.51,43.56,42.30,38.79,31.48$, 29.59, 27.11, 22.77, 20.46; MS (ESI $\left.{ }^{+}\right) m / z 587(\mathrm{M}+\mathrm{H})$; Anal. Calcd for $\mathrm{C}_{33} \mathrm{H}_{35} \mathrm{BrN}_{2} \mathrm{O}_{3}: \mathrm{C}, 67.46$; H, 6.00; N, 4.77. Found: C, 67.45; H, 5.93; N, 4.64.

(2S,3R,4S)-2-(p-tolylamino)- $N$-benzyl-3,4,5,6,7,8-hexahydro-4-(4-methoxyphenyl)-2,7,7-trime thyl-5-oxo-2 $H$-chromene-3-carboxamide $6\{1,1,1,4\}$ :

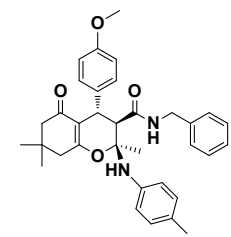

White powder (yield 75\%); m.p. $149-151^{\circ} \mathrm{C} ;{ }^{1} \mathrm{H}$ NMR (300MHz, DMSO-d6, $\mathrm{D}_{2} \mathrm{O}$ exchange) $\delta 8.61\left(\mathrm{~m}, \mathrm{NH}, 1 \mathrm{H}\right.$, exchangeable with $\left.\mathrm{D}_{2} \mathrm{O}\right), 7.16(\mathrm{~d}, J=3.0 \mathrm{~Hz}$, aromatic, $3 \mathrm{H}), 6.98(\mathrm{~d}$, $J=6.0 \mathrm{~Hz}$, aromatic, $4 \mathrm{H}), 6.73-6.82(\mathrm{~m}$, aromatic, $6 \mathrm{H}), 5.75\left(\mathrm{~s}, \mathrm{NH}, 1 \mathrm{H}\right.$, disappeared after $\mathrm{D}_{2} \mathrm{O}$ exchange), 4.40-4.47 (m, $\left.\mathrm{CH}_{2}, 1 \mathrm{H}\right), 3.91-3.99\left(\mathrm{~m}, \mathrm{CH}+\mathrm{CH}_{2}, 2 \mathrm{H}\right), 3.74\left(\mathrm{~s}, \mathrm{CH}_{3}, 3 \mathrm{H}\right), 2.83(\mathrm{~d}, J=$ $9.0 \mathrm{~Hz}, \mathrm{CH}, 1 \mathrm{H}), 2.28-2.44\left(\mathrm{~m}, \mathrm{CH}_{2}, 2 \mathrm{H}\right), 2.18\left(\mathrm{~s}, \mathrm{CH}_{3}, 3 \mathrm{H}\right), 1.94-2.12\left(\mathrm{~m}, \mathrm{CH}_{2}, 2 \mathrm{H}\right), 1.50(\mathrm{~s}$, $\left.\mathrm{CH}_{3}, 3 \mathrm{H}\right), 1.07$ (s, $\left.\mathrm{CH}_{3}, 3 \mathrm{H}\right), 0.94\left(\mathrm{~s}, \mathrm{CH}_{3}, 3 \mathrm{H}\right) ;{ }^{13} \mathrm{C}$ NMR (75MHz, DMSO-d6) $\delta$ 195.3, 170.9, 166.3, 157.7, 157.4, 142.0, 138.5, 135.0, 129.6, 129.2, 128.7, 128.4, 128.2, 127.3, 127.1, 126.9, $126.8,117.4,114.1,113.6,88.0,57.2,55.1,50.5,42.1,42.0,37.5,31.4,29.0,28.5,27.0,20.8 ;$ MS $\left(\mathrm{ESI}^{+}\right) \mathrm{m} / z 539(\mathrm{M}+\mathrm{H})$; Anal. Calcd for $\mathrm{C}_{34} \mathrm{H}_{38} \mathrm{~N}_{2} \mathrm{O}_{4}$ : C, 75.81; H, 7.11; N, 5.20. Found: C, 75.89; $\mathrm{H}, 7.07 ; \mathrm{N}, 5.25$.

$(2 S, 3 R, 4 S)$-2-(p-tolylamino)- $N$-benzyl-3,4,5,6,7,8-hexahydro-2,7,7-trimethyl-4-(4-nitrophenyl)5-oxo-2H-chromene-3-carboxamide $6\{1,1,1,5\}$ :

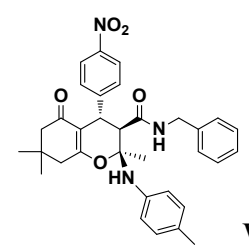

White powder (yield 81\%); m.p. $180-182^{\circ} \mathrm{C} ;{ }^{1} \mathrm{H}$ NMR (300MHz, DMSO- $d 6$ ) $\delta$ 8.63-8.65 (m, NH, 1H), 8.01-8.11 (m, aromatic, $2 \mathrm{H}), 7.30-7.31$ (m, aromatic, $2 \mathrm{H}), 6.99-7.14(\mathrm{~m}$, aromatic, $5 \mathrm{H}), 6.77(\mathrm{~m}$, aromatic, $5 \mathrm{H}), 5.77(\mathrm{~s}, \mathrm{NH}, 1 \mathrm{H}), 4.37-4.42\left(\mathrm{~m}, \mathrm{CH}_{2}, 1 \mathrm{H}\right), 3.92-4.13(\mathrm{~m}$, $\left.\mathrm{CH}+\mathrm{CH}_{2}, 2 \mathrm{H}\right), 2.81-2.87(\mathrm{~m}, \mathrm{CH}, 1 \mathrm{H}), 2.19\left(\mathrm{~s}, \mathrm{CH}_{3}, 3 \mathrm{H}\right), 1.98-2.13\left(\mathrm{~m}, 2 \mathrm{CH}_{2}, 4 \mathrm{H}\right), 1.53\left(\mathrm{~s}, \mathrm{CH}_{3}\right.$, $3 \mathrm{H}), 1.08\left(\mathrm{~s}, \mathrm{CH}_{3}, 3 \mathrm{H}\right), 0.96\left(\mathrm{~s}, \mathrm{CH}_{3}, 3 \mathrm{H}\right) ;{ }^{13} \mathrm{C}$ NMR (75MHz, DMSO-d6) $\delta$ 195.1, 169.8, 151.7, 145.7, 141.4, 138.3, 129.4, 129.1, 128.2, 128.1, 127.9, 127.0, 126.8, 123.2, 117.6, 72.3, 68.8, 56.0,

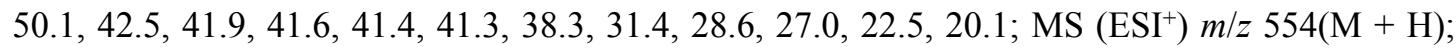
Anal. Calcd for $\mathrm{C}_{33} \mathrm{H}_{35} \mathrm{~N}_{3} \mathrm{O}_{5}$ : C, 71.59; H, 6.37; N, 7.59. Found: C, 71.63; H, 6.31; N, 7.48. $(2 S, 3 R, 4 S)$-2-(p-tolylamino)- $N$-benzyl-4-(4-cyanophenyl)-3,4,5,6,7,8-hexahydro-2,7,7-trimeth yl-5-oxo-2 $H$-chromene-3-carboxamide $6\{1,1,1,6\}$ : 
White powder (yield 81\%); m.p. 160-162 ${ }^{\circ}$; ${ }^{1} \mathrm{H}$ NMR (400 MHz, $\left.\mathrm{CDCl}_{3}\right) \delta 7.48$ $(\mathrm{d}, J=8.2 \mathrm{~Hz}$, aromatic, $2 \mathrm{H}), 7.30(\mathrm{~d}, J=2.0 \mathrm{~Hz}$, aromatic, $3 \mathrm{H}), 7.25(\mathrm{~d}, J=8.1 \mathrm{~Hz}$, aromatic, 2H), $7.04(\mathrm{~d}, J=8.1 \mathrm{~Hz}$, aromatic, 2H), 6.88 (t, $J=7.4 \mathrm{~Hz}$, aromatic, 4H), 5.74 (s, NH, 1H), 5.50 (s, NH, 1H), 4.45 (dd, $\left.J=14.6,6.7 \mathrm{~Hz}, \mathrm{CH}_{2}, 1 \mathrm{H}\right), 4.22$ (d, $\left.J=11.4 \mathrm{~Hz}, \mathrm{CH}, 1 \mathrm{H}\right), 4.11$ (dd, $J=$ 14.6, $\left.4.6 \mathrm{~Hz}, \mathrm{CH}_{2}, 1 \mathrm{H}\right), 2.48-2.38\left(\mathrm{~m}, \mathrm{CH}_{3}, 3 \mathrm{H}\right), 2.29$ (s, $\left.\mathrm{CH}_{3}, 3 \mathrm{H}\right), 2.14$ (dd, $J=34.9,16.6 \mathrm{~Hz}$, $\mathrm{CH} 2,2 \mathrm{H}), 1.64\left(\mathrm{~s}, \mathrm{CH}_{3}, 3 \mathrm{H}\right), 1.15\left(\mathrm{~s}, \mathrm{CH}_{3}, 3 \mathrm{H}\right), 1.05\left(\mathrm{~s}, \mathrm{CH}_{3}, 3 \mathrm{H}\right) .{ }^{13} \mathrm{C} \mathrm{NMR}\left(101 \mathrm{MHz}, \mathrm{CDCl}_{3}\right) \delta$ 196.09, 170.28, 168.10, 149.14, 141.49, 137.01, 132.23, 129.62, 129.34, 128.61, 128.07, 127.74, $127.55,118.92,117.82,113.00,109.85,88.56,58.52,50.41,43.45,42.32,39.35,31.56,29.52$, 27.16, 22.76, 20.48. HRMS: calcd for $\mathrm{C}_{34} \mathrm{H}_{35} \mathrm{~N}_{3} \mathrm{O}_{3}$ 533.2678, found $534.2742[\mathrm{M}+\mathrm{H}]$.

(2S,3R,4S)-2-(p-tolylamino)- $N$-benzyl-3,4,5,6,7,8-hexahydro-4-(4-hydroxyphenyl)-2,7,7-trime thyl-5-oxo-2 $H$-chromene-3-carboxamide $6\{1,1,1,7\}$ :

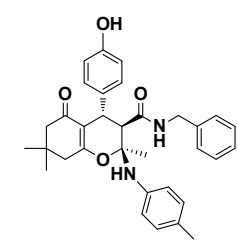

White powder (yield 72\%); m.p. $162-164^{\circ} \mathrm{C} ;{ }^{1} \mathrm{H}$ NMR (400 MHz, DMSO) $\delta 9.15$ (s, OH, 1H), $8.69-8.51$ (m, NH, 1H), $7.20(\mathrm{~d}, J=6.9 \mathrm{~Hz}$, aromatic, 3H), $6.98(\mathrm{~d}, J=8.0 \mathrm{~Hz}$, aromatic, $2 \mathrm{H}), 6.88(\mathrm{~d}, J=8.1 \mathrm{~Hz}$, aromatic, $2 \mathrm{H}), 6.80(\mathrm{~d}, J=6.6 \mathrm{~Hz}$, aromatic, $2 \mathrm{H}), 6.74(\mathrm{~d}, J=$ $8.0 \mathrm{~Hz}$, aromatic, $2 \mathrm{H}), 6.65(\mathrm{~d}, J=8.2 \mathrm{~Hz}$, aromatic, $2 \mathrm{H}), 5.74(\mathrm{~s}, \mathrm{NH}, 1 \mathrm{H}), 4.44$ (dd, $J=15.4,7.0$ $\left.\mathrm{Hz}, \mathrm{CH}_{2}, 1 \mathrm{H}\right), 4.04-3.95$ (m, CH, 1H), 3.89 (d, $\left.J=11.0 \mathrm{~Hz}, \mathrm{CH}_{2}, 1 \mathrm{H}\right), 2.84$ (d, $J=11.2 \mathrm{~Hz}, \mathrm{CH}$, $1 \mathrm{H}), 2.48-2.25\left(\mathrm{~m}, \mathrm{CH}_{2}, 2 \mathrm{H}\right), 2.19\left(\mathrm{~s}, \mathrm{CH}_{3}, 3 \mathrm{H}\right), 2.05$ (dd, $\left.J=35.8,9.8 \mathrm{~Hz}, \mathrm{CH}_{2}, 2 \mathrm{H}\right), 1.51(\mathrm{~s}$, $\left.\mathrm{CH}_{3}, 3 \mathrm{H}\right), 1.08\left(\mathrm{~s}, \mathrm{CH}_{3}, 3 \mathrm{H}\right), 0.95$ (s, $\left.\mathrm{CH}_{3}, 3 \mathrm{H}\right) .{ }^{13} \mathrm{C}$ NMR (101 MHz, DMSO) $\delta$ 195.38, 171.19, 166.12 , 155.94, 155.43, 142.32, 138.75, 133.44, 129.85, 128.80, 128.47, 128.23, 127.29, 127.07, $117.62,115.25,114.58,88.35,57.52,50.79,42.27,41.89,37.78,31.70,29.26,27.20,22.95$, 20.53. MS (ESI+) m/z 525(M+H). Anal. Calcd for $\mathrm{C}_{33} \mathrm{H}_{36} \mathrm{~N}_{2} \mathrm{O}_{4}: \mathrm{C}, 75.55 ; \mathrm{H}, 6.92 ; \mathrm{N}, 5.34$. Found: C, 75.59; H, 6.84; N, 5.41.

$(2 S, 3 R, 4 S)$-2-(p-tolylamino)-N-benzyl-3,4,5,6,7,8-hexahydro-2,7,7-trimethyl-4-(naphthalen-2yl)-5-oxo-2H-chromene-3-carboxamide $6\{1,1,1,8\}$ :

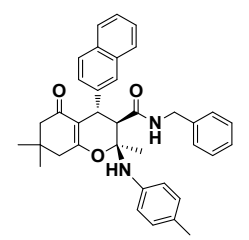

White powder (yield 68\%); m.p. $157-159^{\circ} \mathrm{C} ;{ }^{1} \mathrm{H}$ NMR $\left(400 \mathrm{MHz}, \mathrm{CDCl}_{3}\right) \delta 7.82-$ 7.71 (m, aromatic, 4H), $7.46(\mathrm{dd}, J=5.9 \mathrm{~Hz}, 3.3$, aromatic, 2H), 7.27-7.31 (m, aromatic, $1 \mathrm{H}), 7.05$ $(\mathrm{d}, J=7.0 \mathrm{~Hz}$, aromatic, $3 \mathrm{H}), 6.95-6.81(\mathrm{~m}$, aromatic, $4 \mathrm{H}), 6.51(\mathrm{~d}, J=7.6 \mathrm{~Hz}$, aromatic, $2 \mathrm{H})$, $5.91(\mathrm{~s}, \mathrm{NH}, 1 \mathrm{H}), 5.41(\mathrm{~s}, \mathrm{NH}, 1 \mathrm{H}), 4.34\left(\mathrm{dd}, J=14.5,7.8 \mathrm{~Hz}, \mathrm{CH}_{2}+\mathrm{CH}, 2 \mathrm{H}\right), 4.09-3.98(\mathrm{~m}$, $\left.\mathrm{CH}_{2}, 1 \mathrm{H}\right), 2.63(\mathrm{~d}, J=11.4 \mathrm{~Hz}, \mathrm{CH}, 1 \mathrm{H}), 2.50\left(\mathrm{~d}, J=19.2 \mathrm{~Hz}, \mathrm{CH}_{2}, 2 \mathrm{H}\right), 2.30\left(\mathrm{~s}, \mathrm{CH}_{3}, 3 \mathrm{H}\right), 2.09$ 
(dd, $\left.J=33.8,16.2 \mathrm{~Hz}, \mathrm{CH}_{2}, 2 \mathrm{H}\right), 1.19\left(\mathrm{~s}, \mathrm{CH}_{3}, 3 \mathrm{H}\right), 1.05\left(\mathrm{~d}, J=8.6 \mathrm{~Hz}, \mathrm{CH}_{3}, 3 \mathrm{H}\right) .{ }^{13} \mathrm{C} \mathrm{NMR}(101$ $\left.\mathrm{MHz}, \mathrm{CDCl}_{3}\right) \delta 195.93,170.67,166.86,142.88,141.37,136.66,132.17,128.90,128.80,128.68$, $128.50,127.74,127.49,124.44,118.39,113.73,87.91,59.26,50.56,43.62,42.21,38.71,31.60$, 29.37, 27.22, 22.59. HRMS: calcd for $\mathrm{C}_{37} \mathrm{H}_{38} \mathrm{~N}_{2} \mathrm{O}_{3}$ 558.2882, found 559.2945 [M+H].

(2S,3R,4S)- $N$-(4-methoxybenzyl)-2-(p-tolylamino)-4-(4-chlorophenyl)-3,4,5,6,7,8-hexahydro2,7,7-trimethyl-5-oxo-2 $H$-chromene-3-carboxamide $6\{2,1,1,1\}$ :

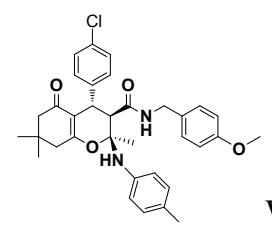

White powder (yield 68\%); m.p. 160-162 ${ }^{\circ}$; ${ }^{1} \mathrm{H}$ NMR (500 MHz, DMSO) $\delta$ $8.54(\mathrm{dd}, J=6.7,4.5 \mathrm{~Hz}, \mathrm{NH}, 1 \mathrm{H}), 7.29(\mathrm{~d}, J=8.3 \mathrm{~Hz}$, aromatic, $2 \mathrm{H}), 7.06(\mathrm{~d}, J=8.1 \mathrm{~Hz}$, aromatic, $2 \mathrm{H}), 6.99(\mathrm{~d}, J=8.1 \mathrm{~Hz}$, aromatic, $2 \mathrm{H}), 6.78-6.75(\mathrm{~m}$, aromatic, $3 \mathrm{H}), 6.71(\mathrm{~d}, J=8.6$ $\mathrm{Hz}$, aromatic, 2H), 5.77 (s, NH, 1H), $4.36\left(\mathrm{dd}, J=14.9,7.2 \mathrm{~Hz}, \mathrm{CH}_{2}, 1 \mathrm{H}\right), 3.98(\mathrm{~d}, J=11.1 \mathrm{~Hz}$, $\mathrm{CH}, 1 \mathrm{H}), 3.90$ (dd, $\left.J=15.0,4.1 \mathrm{~Hz}, \mathrm{CH}_{2}, 1 \mathrm{H}\right), 3.73$ (d, $\left.J=12.9 \mathrm{~Hz}, \mathrm{CH}_{3}, 3 \mathrm{H}\right), 2.81-2.54$ (m, $\mathrm{CH}_{2}$, 1H), 2.47 (s, $\left.\mathrm{CH}_{2}, 1 \mathrm{H}\right), 2.19$ (s, $\left.\mathrm{CH}_{3}, 3 \mathrm{H}\right), 2.11$ (d, $\left.J=16.4 \mathrm{~Hz}, \mathrm{CH}_{2}, 1 \mathrm{H}\right), 2.00$ (d, $J=16.4 \mathrm{~Hz}$, $\left.\mathrm{CH}_{2}, 1 \mathrm{H}\right), 1.50\left(\mathrm{~s}, \mathrm{CH}_{3}, 3 \mathrm{H}\right), 1.07$ (s, $\left.\mathrm{CH}_{3}, 3 \mathrm{H}\right), 0.95\left(\mathrm{~s}, \mathrm{CH}_{3}, 3 \mathrm{H}\right) .{ }^{13} \mathrm{C}$ NMR (125 MHz, DMSO) $\delta$ $195.50,170.59,167.12,158.68,142.61,142.28,130.83$, 130.70, 129.91, 129.76, 128.74, 128.48, 117.87, 113.92, 113.66, 88.51, 56.99, 55.48, 50.73, 41.83, 38.20, 31.86, 29.19, 27.39, 22.96, 20.59.MS $\left(\mathrm{ESI}^{+}\right) \mathrm{m} / z$ 573(M+H); Anal. Calcd for $\mathrm{C}_{34} \mathrm{H}_{37} \mathrm{ClN}_{2} \mathrm{O}_{4}$ : C, 71.25; H, 6.51; N, 4.89 . Found: C, 71.29; H, 6.50; N, 4.75.

(2S,3R,4S)- $N$-(4-fluorobenzyl)-2-(p-tolylamino)-4-(4-chlorophenyl)-3,4,5,6,7,8-hexahydro-2,7, 7-trimethyl-5-oxo-2H-chromene-3-carboxamide 6\{3,1,1,1\}:

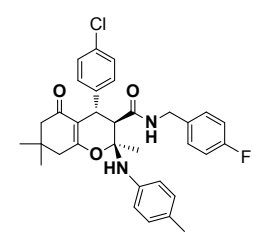

White powder (yield 59\%); m.p. $160-162^{\circ} \mathrm{C}$; ${ }^{1} \mathrm{H}$ NMR (500 MHz, DMSO) $\delta$ $8.63(\mathrm{~s}, \mathrm{NH}, 1 \mathrm{H}), 7.29$ (d, $J=7.5 \mathrm{~Hz}$, aromatic, 2H), 7.09-6.95 (m, aromatic, 6H), 6.88-6.72 (m, aromatic, $4 \mathrm{H}), 5.74(\mathrm{~s}, \mathrm{NH}, 1 \mathrm{H}), 4.41\left(\mathrm{dd}, J=15.1,7.0 \mathrm{~Hz}, \mathrm{CH}_{2}, 1 \mathrm{H}\right), 3.96(\mathrm{dd}, J=14.8,4.0 \mathrm{~Hz}$, $\left.\mathrm{CH}+\mathrm{CH}_{2}, 2 \mathrm{H}\right), 2.81(\mathrm{~d}, J=11.1 \mathrm{~Hz}, \mathrm{CH}, 1 \mathrm{H}), 2.49-2.28\left(\mathrm{~m}, \mathrm{CH}_{2}, 2 \mathrm{H}\right), 2.19\left(\mathrm{~s}, \mathrm{CH}_{3}, 3 \mathrm{H}\right), 2.06$ (dd, J=55.0, 16.4 Hz, $\left.\mathrm{CH}_{2}, 2 \mathrm{H}\right), 1.51\left(\mathrm{~s}, \mathrm{CH}_{3}, 3 \mathrm{H}\right), 1.07$ (s, $\left.\mathrm{CH}_{3}, 3 \mathrm{H}\right), 0.96$ (d, J=12.0 Hz, $\mathrm{CH}_{3}$, $3 \mathrm{H}) ;{ }^{13} \mathrm{C}$ NMR (125 MHz, DMSO) $\delta 195.52,170.76,167.15,162.53,160.60,142.58,142.23$, 135.05, 130.84, 129.92, 129.77, 129.46, 129.39, 128.48, 117.90, 115.28, 115.11, 113.62, 88.48, 57.02, 50.71, 41.98, 41.67, 38.21, 31.86, 29.18, 27.36, 22.98, 20.59; $\mathrm{MS}\left(\mathrm{ESI}^{+}\right) \mathrm{m} / \mathrm{z}$ 561(M + H); Anal. Calcd for $\mathrm{C}_{33} \mathrm{H}_{34} \mathrm{ClFN}_{2} \mathrm{O}_{3}: \mathrm{C}, 70.64 ; \mathrm{H}, 6.11 ; \mathrm{N}, 4.99$. Found: C, 70.65; H, 6.18; N, 4.92.

(2S,3R,4S)- $N$-(2-fluorobenzyl)-2-(p-tolylamino)-4-(4-chlorophenyl)-3,4,5,6,7,8-hexahydro-2,7, 7-trimethyl-5-oxo-2H-chromene-3-carboxamide 6\{4,1,1,1\}: 
White powder (yield 76\%); m.p. 166-168 ${ }^{\circ}$; ${ }^{1} \mathrm{H}$ NMR (500MHz, DMSO- $d 6$ ) $\delta$ $8.65(\mathrm{~m}, \mathrm{NH}, 1 \mathrm{H}), 7.24-7.29(\mathrm{~m}$, aromatic, $3 \mathrm{H}), 6.98-7.12$ (m, aromatic, $6 \mathrm{H}), 6.75(\mathrm{~d}, J=10 \mathrm{~Hz}$, aromatic, $2 \mathrm{H}), 6.60-6.63(\mathrm{~m}$, aromatic, $1 \mathrm{H}), 5.72(\mathrm{~s}, \mathrm{NH}, 1 \mathrm{H}), 4.36-4.40\left(\mathrm{~m}, \mathrm{CH}_{2}, 1 \mathrm{H}\right), 3.95-4.11$ (m, $\left.\mathrm{CH}+\mathrm{CH}_{2}, 2 \mathrm{H}\right), 2.83(\mathrm{~d}, J=10 \mathrm{~Hz}, \mathrm{CH}, 1 \mathrm{H}), 2.31-2.47\left(\mathrm{~m}, \mathrm{CH}_{2}, 2 \mathrm{H}\right), 2.19\left(\mathrm{~s}, \mathrm{CH}_{3}, 3 \mathrm{H}\right)$, 1.99-2.16 (m, $\left.\mathrm{CH}_{2}, 2 \mathrm{H}\right), 1.51\left(\mathrm{~s}, \mathrm{CH}_{3}, 3 \mathrm{H}\right), 1.07\left(\mathrm{~s}, \mathrm{CH}_{3}, 3 \mathrm{H}\right), 0.95\left(\mathrm{~s}, \mathrm{CH}_{3}, 3 \mathrm{H}\right) ;{ }^{13} \mathrm{C}$ NMR $(126$ MHz, DMSO) $\delta 195.51,170.95,167.19,161.29,159.35,142.52,130.81,130.18,129.91,129.84$, $129.66,129.47,129.40,128.44,125.49,125.38,124.40,121.50,117.89,115.50,115.33,113.62$, $88.49,56.96,50.72,41.99,38.16,36.25,36.22,31.87,29.18,28.41,27.38,22.91,20.59$; MS $\left(\mathrm{ESI}^{+}\right) \mathrm{m} / z$ 561(M+H); Anal. Calcd for $\mathrm{C}_{33} \mathrm{H}_{34} \mathrm{ClFN}_{2} \mathrm{O}_{3}: \mathrm{C}, 70.64 ; \mathrm{H}, 6.11 ; \mathrm{N}, 4.99$. Found: $\mathrm{C}$, 70.72; H, 6.10; N, 4.95 .

(2S,3R,4S)-2-(p-tolylamino)-4-(4-chlorophenyl)-3,4,5,6,7,8-hexahydro-2,7,7-trimethyl-5-oxo$N$-p-tolyl-2H-chromene-3-carboxamide $6\{5,1,1,1\}$ :

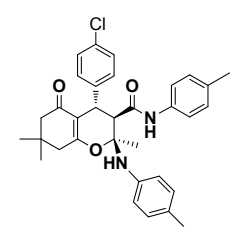

White powder (yield 83\%); m.p. 185-187 ${ }^{\circ} \mathrm{C} ;{ }^{1} \mathrm{H}$ NMR (500MHz, DMSO- $\left.d 6\right) \delta$ $9.95(\mathrm{~m}, \mathrm{NH}, 1 \mathrm{H}), \quad 7.26-7.47$ (m, aromatic, 4H), 6.98-7.19 (m, aromatic, $6 \mathrm{H}), 6.78$ (d, J=5 Hz, aromatic, 2H), $5.73(\mathrm{~s}, \mathrm{OH}, 1 \mathrm{H}), 4.05-4.07(\mathrm{~m}, \mathrm{CH}, 1 \mathrm{H}), 2.92(\mathrm{~d}, J=10 \mathrm{~Hz}, \mathrm{CH}, 1 \mathrm{H}), 2.28-2.36(\mathrm{~m}$, $\left.\mathrm{CH}_{2}, 2 \mathrm{H}\right), 2.19$ (s, $\left.\mathrm{CH}_{3}, 3 \mathrm{H}\right), 2.15\left(\mathrm{~s}, \mathrm{CH}_{3}, 3 \mathrm{H}\right), 1.99-2.12\left(\mathrm{~m}, \mathrm{CH}_{2}, 2 \mathrm{H}\right), 1.58\left(\mathrm{~s}, \mathrm{CH}_{3}, 3 \mathrm{H}\right), 1.04$ $\left(\mathrm{s}, \mathrm{CH}_{3}, 3 \mathrm{H}\right), 0.96\left(\mathrm{~s}, \mathrm{CH}_{3}, 3 \mathrm{H}\right) ;{ }^{13} \mathrm{C}$ NMR (126 MHz, DMSO) $\delta 195.30,169.64,167.54,142.72$, $142.18,135.42,133.15,129.90,129.58,128.50,127.23,123.54,123.37,120.59,118.01,113.56$, 88.29, 57.83, 50.69, 42.02, 38.30, 31.94, 29.11, 27.51, 23.01, 20.90, 20.60. MS (ESI $\left.{ }^{+}\right) \mathrm{m} / z$ 543(M $+\mathrm{H}$ ); Anal. Calcd for $\mathrm{C}_{33} \mathrm{H}_{35} \mathrm{ClN}_{2} \mathrm{O}_{3}$ : C, 72.98; H, 6.50; N, 5.16. Found: C, 72.93; H, 6.58; N, 6.63 .

$(2 S, 3 R, 4 S)$-( $p$-tolylamino)- $N$-butyl-4-(4-chlorophenyl)-3,4,5,6,7,8-hexahydro-2,7,7-trimethyl5-0xo-2H-chromene-3-carboxamide 6\{6,1,1,1\}:

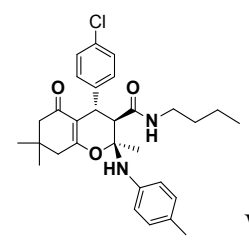

White powder (yield 71\%); m.p. 146-148 ${ }^{\circ} \mathrm{C} ;{ }^{1} \mathrm{H}$ NMR (500MHz, DMSO- $\left.d 6\right) \delta$ 8.05 (m, NH, 1H), 7.28 (d, $J=10 \mathrm{~Hz}$, aromatic, 2H), 6.97-7.04 (m, aromatic, 4H), 6.74 (d, J=5 Hz, aromatic, $2 \mathrm{H}), 5.83(\mathrm{~s}, \mathrm{NH}, 1 \mathrm{H}), 3.92(\mathrm{~d}, J=10 \mathrm{~Hz}, \mathrm{CH}, 1 \mathrm{H}), 3.07$ (d, $J=5 \mathrm{~Hz}, \mathrm{CH}, 1 \mathrm{H}), 2.68-2.83$ (m, $\left.\mathrm{CH}_{2}, 2 \mathrm{H}\right), 2.31-2.47$ (m, $\left.\mathrm{CH}_{2}, 2 \mathrm{H}\right), 2.19\left(\mathrm{~s}, \mathrm{CH}_{3}, 3 \mathrm{H}\right), 1.99-2.13\left(\mathrm{~m}, \mathrm{CH}_{2}, 2 \mathrm{H}\right), 1.49\left(\mathrm{~s}, \mathrm{CH}_{3}\right.$, $3 \mathrm{H}), 1.11-1.16\left(\mathrm{~m}, \mathrm{CH}_{2}, 2 \mathrm{H}\right), 1.08\left(\mathrm{~s}, \mathrm{CH}_{3}, 3 \mathrm{H}\right), 0.88-0.95\left(\mathrm{~m}, \mathrm{CH}_{2}+\mathrm{CH}_{3}, 5 \mathrm{H}\right), 0.73-0.76\left(\mathrm{~m}, \mathrm{CH}_{3}\right.$, 
$3 \mathrm{H}) ;{ }^{13} \mathrm{C}$ NMR (125MHz, DMSO- $\left.d 6\right) \delta$ 195.5, 170.6, 167.3, 142.7, 142.4, 30.7, 130.4, 19.9, 129.5, 128.3, 117.8, 113.6, 88.5, 57.0, 50.7, 42.0, 38.6, 38.2, 31.9, 31.2, 29.2, 27.4, 22.9, 20.6, 19.6, 14.0; MS (ESI $\left.{ }^{+}\right) \mathrm{m} / z$ 509(M+ H); Anal. Calcd for $\mathrm{C}_{30} \mathrm{H}_{37} \mathrm{ClN}_{2} \mathrm{O}_{3}: \mathrm{C}, 70.78 ; \mathrm{H}, 7.33 ; \mathrm{N}, 5.50$. Found: C, 70.71; H, 7.39; N, 5.57.

(2S,3R,4S)-N-benzyl-4-(4-chlorophenyl)-3,4,5,6,7,8-hexahydro-2,7,7-trimethyl-5-oxo-2-(phen ylamino)-2H-chromene-3-carboxamide $6\{1,1,2,1\}$ :

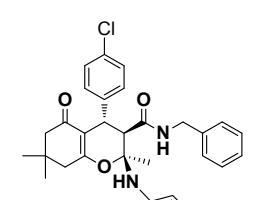

White powder (yield 76\%); m.p. $158-160^{\circ} \mathrm{C} ;{ }^{1} \mathrm{H}$ NMR (500MHz, DMSO- $\left.d 6\right) \delta$ 8.64-8.66 (m, NH, 1H), 7.28-7.31 (m, aromatic, $2 \mathrm{H}), 7.16-7.21$ (m, aromatic, $5 \mathrm{H}), 7.08(\mathrm{~d}, J=10$ $\mathrm{Hz}$, aromatic, 2H), 6.76-6.87 (m, aromatic, 5H), $5.91(\mathrm{~s}, \mathrm{NH}, 1 \mathrm{H}), 4.43-4.47\left(\mathrm{~m}, \mathrm{CH}_{2}, 1 \mathrm{H}\right)$, 3.96-4.00 (m, CH+CH $2,2 \mathrm{H}), 2.85(\mathrm{~d}, J=15 \mathrm{~Hz}, \mathrm{CH}, 1 \mathrm{H}), 2.32-2.48\left(\mathrm{~m}, \mathrm{CH}_{2}, 2 \mathrm{H}\right), 1.98-2.13(\mathrm{~m}$, $\left.\mathrm{CH}_{2}, 2 \mathrm{H}\right), 1.56\left(\mathrm{~s}, \mathrm{CH}_{3}, 3 \mathrm{H}\right), 1.08\left(\mathrm{~s}, \mathrm{CH}_{3}, 3 \mathrm{H}\right), 0.97\left(\mathrm{~s}, \mathrm{CH}_{3}, 3 \mathrm{H}\right) ;{ }^{13} \mathrm{C}$ NMR (125MHz, DMSO-d6) $\delta$ 195.5, 170.7, 167.1, 144.8, 142.5, 138.7, 130.9, 129.5, 128.5, 127.4, 127.3, 119.7,

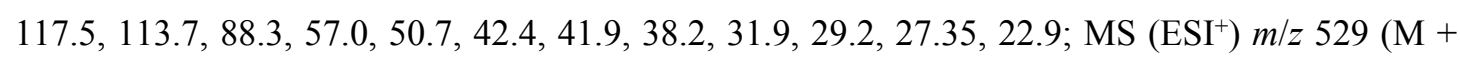
H); Anal. Calcd for $\mathrm{C}_{32} \mathrm{H}_{33} \mathrm{ClN}_{2} \mathrm{O}_{3}: \mathrm{C}, 72.65 ; \mathrm{H}, 6.29 ; \mathrm{N}, 5.29$. Found: C, 72.67; H, 6.20; N, 5.34.

$(2 S, 3 R, 4 S)$-2-(4-chlorophenylamino)- $N$-benzyl-4-(4-chlorophenyl)-3,4,5,6,7,8-hexahydro-2,7, 7-trimethyl-5-oxo-2H-chromene-3-carboxamide $6\{1,1,3,1\}$ :

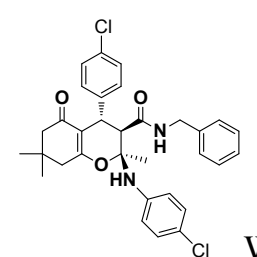

White powder (yield 71\%); m.p. $168-171{ }^{\circ} \mathrm{C}$; ${ }^{1} \mathrm{H}$ NMR $(500 \mathrm{MHz}$, DMSO- $d 6) \delta$ 8.63-8.65 (m, NH, 1H), 7.27-7.31 (m, aromatic, 2H), 7.21 (m, aromatic, 5H), 7.07 (d, J=5 Hz, aromatic, $2 \mathrm{H}), 6.89(\mathrm{~d}, J=10 \mathrm{~Hz}$, aromatic, $2 \mathrm{H}), 6.78(\mathrm{~d}, J=5 \mathrm{~Hz}$, aromatic, $2 \mathrm{H}), 6.06(\mathrm{~s}, \mathrm{NH}, 1 \mathrm{H})$, 4.43-4.47 (m, $\left.\mathrm{CH}_{2}, 1 \mathrm{H}\right), 3.96-4.02\left(\mathrm{~m}, \mathrm{CH}+\mathrm{CH}_{2}, 2 \mathrm{H}\right), 2.85$ (d, $\left.J=10 \mathrm{~Hz}, \mathrm{CH}, 1 \mathrm{H}\right), 2.30-2.48$ (m, $\left.\mathrm{CH}_{2}, 2 \mathrm{H}\right), 1.99-2.12\left(\mathrm{~m}, \mathrm{CH}_{2}, 2 \mathrm{H}\right), 1.56\left(\mathrm{~s}, \mathrm{CH}_{3}, 3 \mathrm{H}\right), 1.07\left(\mathrm{~s}, \mathrm{CH}_{3}, 3 \mathrm{H}\right), 0.92\left(\mathrm{~s}, \mathrm{CH}_{3}, 3 \mathrm{H}\right) ;{ }^{13} \mathrm{C}$ NMR (125MHz, DMSO-d6) $\delta$ 195.5, 170.6, 166.9, 143.7, 142.4, 138.7, 130.9, 129.8, 129.2, 18.5, 128.4, 127.3, 127.1, 123.2, 118.8, 113.7, 88.0, 56.9, 50.7, 42.5, 41.8, 39.5, 38.1, 32.0, 29.0, 27.5, 26.8, 22.8; $\mathrm{MS}\left(\mathrm{ESI}^{+}\right) \mathrm{m} / \mathrm{z} 563(\mathrm{M}+\mathrm{H})$; Anal. Calcd for $\mathrm{C}_{32} \mathrm{H}_{32} \mathrm{Cl}_{2} \mathrm{~N}_{2} \mathrm{O}_{3}: \mathrm{C}, 68.20 ; \mathrm{H}, 5.72 ; \mathrm{N}$, 4.97. Found: C, 68.24; H, 5.76; N, 4.90.

(2S,3R,4S)-2-(o-tolylamino)- $N$-benzyl-4-(4-chlorophenyl)-3,4,5,6,7,8-hexahydro-2,7,7-trimeth yl-5-oxo-2H-chromene-3-carboxamide $6\{1,1,4,1\}$ : 


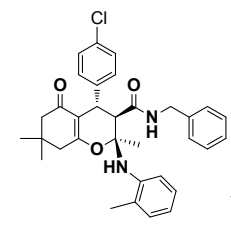

White powder (yield 46\%); m.p. 170-173 ${ }^{\circ} \mathrm{C} ;{ }^{1} \mathrm{H}$ NMR (500MHz, DMSO- $d 6$ ) $\delta 8.69$ $(\mathrm{m}, \mathrm{NH}, 1 \mathrm{H}), 7.31(\mathrm{~d}, J=10 \mathrm{~Hz}$, aromatic, $2 \mathrm{H}), 7.11-7.20(\mathrm{~m}$, aromatic, 3H), 7.04-7.09 (m, aromatic, 5H), 6.81-6.86 (m, aromatic, $2 \mathrm{H}), 6.69(\mathrm{~s}$, aromatic, $1 \mathrm{H}), 5.88(\mathrm{~s}, \mathrm{NH}, 1 \mathrm{H}), 4.41-4.45$ (m, $\mathrm{CH}, 1 \mathrm{H}), 4.00-4.05\left(\mathrm{~m}, \mathrm{CH}_{2}, 2 \mathrm{H}\right), 2.51-2.88(\mathrm{~m}, \mathrm{CH}, 1 \mathrm{H}), 2.33-2.48\left(\mathrm{~m}, \mathrm{CH}_{2}, 2 \mathrm{H}\right), 1.98-2.21$ (m, $\left.\mathrm{CH}_{3}+\mathrm{CH}_{2}, 5 \mathrm{H}\right), 1.51\left(\mathrm{~s}, \mathrm{CH}_{3}, 3 \mathrm{H}\right), 1.08\left(\mathrm{~s}, \mathrm{CH}_{3}, 3 \mathrm{H}\right), 0.97\left(\mathrm{~s}, \mathrm{CH}_{3}, 3 \mathrm{H}\right) ;{ }^{13} \mathrm{C}$ NMR $(125 \mathrm{MHz}$, DMSO-d6) $\delta$ 195.6, 171.0, 167.1, 143.0, 142.6, 138.7, 130.9, 130.8, 129.8, 128.5, 127.7, 127.5, 127.3, 127.0, 124.9, 119.4, 116.5, 113.7, 88.4, 57.2, 50.7, 42.5, 41.9, 38.3, 31.9, 29.2, 27.3, 23.2, 18.0; MS $\left(\mathrm{ESI}^{+}\right) \mathrm{m} / z 543(\mathrm{M}+\mathrm{H})$; Anal. Calcd for $\mathrm{C}_{33} \mathrm{H}_{35} \mathrm{ClN}_{2} \mathrm{O}_{3}: \mathrm{C}, 72.98 ; \mathrm{H}, 6.50 ; \mathrm{N}, 5.16$. Found: C, 72.92; H, 6.59; N, 5.22 .

(2S,3R,4S)-2-(p-tolylamino)- $N$-benzyl-4-(4-chlorophenyl)-3,4,5,6,7,8-hexahydro-2-methyl-5-o xo-2H-chromene-3-carboxamide $6\{1,2,1,1\}$ :

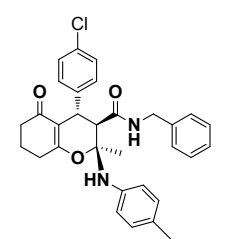

White powder (yield 49\%); m.p. $163-165^{\circ} \mathrm{C} ;{ }^{1} \mathrm{H}$ NMR (400 MHz, DMSO) $\delta 8.70-$ $8.57(\mathrm{~m}, \mathrm{NH}, 1 \mathrm{H}), 7.28(\mathrm{~d}, J=8.3 \mathrm{~Hz}$, aromatic, $2 \mathrm{H}), 7.24-7.19$ (m, aromatic, 3H), $7.10(\mathrm{~d}, J=$ $8.2 \mathrm{~Hz}$, aromatic, $2 \mathrm{H}), 6.99(\mathrm{~d}, J=8.1 \mathrm{~Hz}$, aromatic, $2 \mathrm{H}), 6.86-6.73(\mathrm{~m}$, aromatic, $4 \mathrm{H}), 5.77(\mathrm{~s}$, NH, 1H), $4.44\left(\mathrm{dd}, J=15.3,7.1 \mathrm{~Hz}, \mathrm{CH}_{2}, 1 \mathrm{H}\right), 3.99\left(\mathrm{dd}, J=15.0,3.9 \mathrm{~Hz}, \mathrm{CH}+\mathrm{CH}_{2}, 2 \mathrm{H}\right), 2.79(\mathrm{~d}$, $J=11.2 \mathrm{~Hz}, \mathrm{CH}, 1 \mathrm{H}), 2.57\left(\mathrm{~d}, J=17.8 \mathrm{~Hz}, \mathrm{CH}_{2}, 1 \mathrm{H}\right), 2.42\left(\mathrm{dd}, J=16.5,7.8 \mathrm{~Hz}, \mathrm{CH}_{2}, 1 \mathrm{H}\right), 2.22$ $2.10\left(\mathrm{~m}, \mathrm{CH}_{3}+\mathrm{CH}_{2}, 5 \mathrm{H}\right), 1.90\left(\mathrm{~d}, J=4.8 \mathrm{~Hz}, \mathrm{CH}_{2}, 2 \mathrm{H}\right), 1.53\left(\mathrm{~d}, J=17.4 \mathrm{~Hz}, \mathrm{CH}_{3}, 3 \mathrm{H}\right) ;{ }^{13} \mathrm{C} \mathrm{NMR}$ $\left(100 \mathrm{MHz}, \mathrm{CDCl}_{3}\right) \delta 196.01,170.68,168.80,141.70,141.61,136.80,132.07,129.52,129.08$, $128.73,128.67,128.45,127.70,127.54,117.77,115.07,88.21,59.60,43.61,38.62,36.76,28.68$, 22.79, 20.44, 19.99; HRMS: calcd for $\mathrm{C}_{31} \mathrm{H}_{31} \mathrm{ClN}_{2} \mathrm{O}_{3}$ 514.2023, found 515.2091 [M+H].

$(2 S, 3 R, 4 S)$-( $p$-tolylamino)- $N$-benzyl-3,4,5,6,7,8-hexahydro-2-methyl-5-oxo-4-phenyl-2H-chro mene-3-carboxamide, $6\{1,2,1,2\}$ :

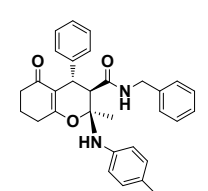

White powder (yield 38\%); m.p. 157.8-161.5 ${ }^{\circ} \mathrm{C} ;{ }^{1} \mathrm{H} \mathrm{NMR}\left(400 \mathrm{MHz}, \mathrm{CDCl}_{3}\right) \delta 7.24$ - $7.14(\mathrm{~m}$, aromatic, $8 \mathrm{H}), 7.01(\mathrm{~d}, J=7.9 \mathrm{~Hz}$, aromatic, $2 \mathrm{H}), 6.86(\mathrm{~d}, J=7.6 \mathrm{~Hz}$, aromatic, 4H), $5.83(\mathrm{~s}, \mathrm{NH}, 1 \mathrm{H}), 5.33(\mathrm{~s}, \mathrm{NH}, 1 \mathrm{H}), 4.32\left(\mathrm{dd}, J=14.8,6.1 \mathrm{~Hz}, \mathrm{CH}_{2}, 1 \mathrm{H}\right), 4.18(\mathrm{dd}, J=14.0,7.7$ $\left.\mathrm{Hz}, \mathrm{CH}+\mathrm{CH}_{2}, 2 \mathrm{H}\right), 2.61-2.39\left(\mathrm{~m}, \mathrm{CH}_{3}, 3 \mathrm{H}\right), 2.26\left(\mathrm{~s}, \mathrm{CH}_{2}+\mathrm{CH}_{3}, 5 \mathrm{H}\right), 1.97\left(\mathrm{~s}, \mathrm{CH}_{2}, 2 \mathrm{H}\right), 1.61(\mathrm{~s}$, $\left.\mathrm{CH}_{3}, 3 \mathrm{H}\right) ;{ }^{13} \mathrm{C}$ NMR $\left(100 \mathrm{MHz}, \mathrm{CDCl}_{3}\right) \delta 195.96,171.07,168.40,143.06,141.88,136.92,129.48$, $128.88,128.56,127.58,127.07,126.39,117.72,115.54,88.17,59.81,43.61,39.18,36.81,28.69$, 22.76, 20.44, 20.02; HRMS: calcd for $\mathrm{C}_{31} \mathrm{H}_{32} \mathrm{~N}_{2} \mathrm{O}_{3}$ 480.2413, found 481.2474 [M+H]. 
(2S,3R,4S)-2-(p-tolylamino)- $N$-benzyl-3,4,5,6,7,8-hexahydro-4-(4-methoxyphenyl)-2-methyl-5 -oxo-2H-chromene-3-carboxamide $6\{1,2,1,4\}$ :

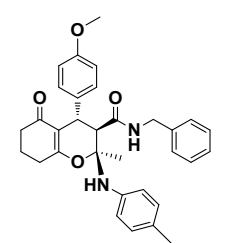

White powder (yield 41\%); m.p. 161.9-163.1 ${ }^{\circ} \mathrm{C} ;{ }^{1} \mathrm{H}$ NMR (400 MHz, $\mathrm{CDCl}_{3}$ ) $\delta$ 7.27-7.20 (m, aromatic, 3H), $7.11(\mathrm{~d}, J=8.3$, aromatic, $2 \mathrm{H}), 7.03(\mathrm{~d}, J=8.0 \mathrm{~Hz}$, aromatic, $2 \mathrm{H}), 6.88$ (d, $J=8.2 \mathrm{~Hz}$, aromatic, 4H), 6.79 (d, $J=8.3 \mathrm{~Hz}$, aromatic, $2 \mathrm{H}), 5.84(\mathrm{~s}, \mathrm{NH}, 1 \mathrm{H}), 5.44(\mathrm{~d}, J=5.2 \mathrm{~Hz}$, $\mathrm{NH}, 1 \mathrm{H}), 4.41\left(\mathrm{dd}, J=14.7 \mathrm{~Hz}, 6.3, \mathrm{CH}_{2}, 1 \mathrm{H}\right), 4.20-4.10\left(\mathrm{~m}, \mathrm{CH}+\mathrm{CH}_{2}, 2 \mathrm{H}\right), 3.78(\mathrm{~d}, J=7.2 \mathrm{~Hz}$, $\left.\mathrm{CH}_{3}, 3 \mathrm{H}\right), 2.64-2.40\left(\mathrm{~m}, \mathrm{CH}_{3}, 3 \mathrm{H}\right), 2.33-2.15\left(\mathrm{~m}, \mathrm{CH}_{3}+\mathrm{CH}_{2}, 5 \mathrm{H}\right), 1.97$ (dd, J=12.4, $9.1 \mathrm{~Hz}$, $\left.\mathrm{CH}_{2}, 2 \mathrm{H}\right), 1.63\left(\mathrm{~s}, \mathrm{CH}_{3}, 3 \mathrm{H}\right) ;{ }^{13} \mathrm{C} \mathrm{NMR}\left(100 \mathrm{MHz}, \mathrm{CDCl}_{3}\right) \delta 196.13,171.19,168.30,158.09$, $141.91,137.05,134.91,129.48,128.84,128.51,128.07,127.58,117.72,115.73,113.99,88.23$, 59.83, 55.09, 43.54, 38.36, 36.84, 28.70, 22.77, 20.44, 20.02; HRMS: calcd for $\mathrm{C}_{32} \mathrm{H}_{34} \mathrm{~N}_{2} \mathrm{O}_{4}$ 510.2519, found $511.2578[\mathrm{M}+\mathrm{H}]$.

(2S,3R,4S)-2-(4-chlorophenylamino)- $N$-benzyl-4-(4-chlorophenyl)-3,4,5,6,7,8-hexahydro-2-m ethyl-5-oxo-2H-chromene-3-carboxamide, $6\{1,2,3,1\}$ :

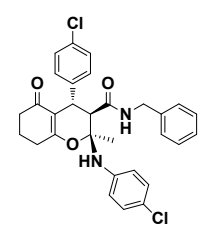

White powder (yield 56\%); m.p. 177.7-180.1 ${ }^{\circ} \mathrm{C}$; ${ }^{1} \mathrm{H}$ NMR (400 MHz, DMSO) $\delta$ 8.70-8.61 (m, NH, 1H), 7.28 (s, aromatic, $1 \mathrm{H}), 7.20(\mathrm{~d}, J=7.5 \mathrm{~Hz}$, aromatic, $6 \mathrm{H}), 7.08(\mathrm{~d}, J=8.0$ $\mathrm{Hz}$, aromatic, $2 \mathrm{H}), 6.88(\mathrm{~d}, J=8.5 \mathrm{~Hz}$, aromatic, $2 \mathrm{H}), 6.81(\mathrm{~d}, J=4.0 \mathrm{~Hz}$, aromatic, $2 \mathrm{H}), 6.06(\mathrm{~s}$, $\mathrm{NH}, 1 \mathrm{H}), 4.43\left(\mathrm{dd}, J=15.2,7.1 \mathrm{~Hz}, \mathrm{CH}_{2}, 1 \mathrm{H}\right), 4.03-3.95\left(\mathrm{~m}, \mathrm{CH}+\mathrm{CH}_{2}, 2 \mathrm{H}\right), 2.80(\mathrm{~d}, J=11.5 \mathrm{~Hz}$, $\mathrm{CH}, 1 \mathrm{H}), 2.55-2.35\left(\mathrm{~m}, \mathrm{CH}_{3}+\mathrm{CH}_{2}, 5 \mathrm{H}\right), 2.17-2.09(\mathrm{~m}, 2 \mathrm{H}), 1.88\left(\mathrm{~s}, \mathrm{CH}_{2}, 2 \mathrm{H}\right), 1.55\left(\mathrm{~s}, \mathrm{CH}_{3}, 3 \mathrm{H}\right)$; ${ }^{13} \mathrm{C}$ NMR (100 MHz, DMSO) $\delta$ 195.59, 170.56, 168.62, 143.65, 142.39, 138.63, 130.84, 130.83, $129.61,129.20,128.49,128.42,127.48,127.28,123.27,118.90,114.91,87.82,56.82,42.41$, 37.87, 36.90, 28.43, 22.75, 20.07; HRMS: calcd for $\mathrm{C}_{30} \mathrm{H}_{28} \mathrm{Cl}_{2} \mathrm{~N}_{2} \mathrm{O}_{3}$ 534.1477, found 535.1537 $[\mathrm{M}+\mathrm{H}]$.

(2S,3R,4S)-2-(p-tolylamino)- $N$-benzyl-2,3,4,5-tetrahydro-4-(4-chlorophenyl)-2-methyl-5-oxop yrano[3,2-c]chromene-3-carboxamide, $6\{1,3,1,1\}$ :

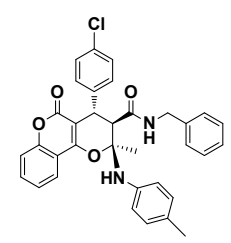

White powder (yield 65\%); m.p. 147.3-148.2 ${ }^{\circ} \mathrm{C} ;{ }^{1} \mathrm{H}$ NMR (400 MHz, $\mathrm{CDCl}_{3}$ ) $\delta$ $7.97(\mathrm{~d}, J=7.9 \mathrm{~Hz}$, aromatic, $1 \mathrm{H}), 7.52(\mathrm{t}, J=7.7 \mathrm{~Hz}$, aromatic, $1 \mathrm{H}), 7.35$ (t, $J=7.6 \mathrm{~Hz}$, aromatic, 1H), 7.28 (s, aromatic, 4H), $7.21(\mathrm{~d}, J=8.1 \mathrm{~Hz}$, aromatic, $2 \mathrm{H}), 7.14(\mathrm{~d}, J=8.0 \mathrm{~Hz}$, aromatic, $2 \mathrm{H})$, $6.98(\mathrm{~d}, J=8.0 \mathrm{~Hz}$, aromatic, 2H), $6.86(\mathrm{~d}, J=8.2 \mathrm{~Hz}$, aromatic, 4H), $5.90(\mathrm{~s}, \mathrm{NH}, 1 \mathrm{H}), 5.56(\mathrm{~s}$, 
NH, 1H), $4.52-4.42\left(\mathrm{~m}, \mathrm{CH}+\mathrm{CH}_{2}, 2 \mathrm{H}\right), 4.16\left(\mathrm{dd}, J=14.5,4.0 \mathrm{~Hz}, \mathrm{CH}_{2}, 1 \mathrm{H}\right), 2.64(\mathrm{~d}, J=11.5$ $\mathrm{Hz}, \mathrm{CH}, 1 \mathrm{H}), 2.22\left(\mathrm{~s}, \mathrm{CH}_{3}, 3 \mathrm{H}\right), 1.77\left(\mathrm{~s}, \mathrm{CH}_{3}, 3 \mathrm{H}\right) ;{ }^{13} \mathrm{C} \mathrm{NMR}\left(100 \mathrm{MHz}, \mathrm{CDCl}_{3}\right) \delta 170.13,160.55$, 157.98, 153.03, 140.97, 139.77, 132.77, 131.72, 129.56, 128.99, 128.71, 127.78, 127.53, 123.91, $122.56,118.56,116.75,115.65,104.44,89.72,43.73,39.62,22.86,20.43$; HRMS: calcd for $\mathrm{C}_{34} \mathrm{H}_{29} \mathrm{ClN}_{2} \mathrm{O}_{4} 564.1816$, found $565.1876[\mathrm{M}+\mathrm{H}]$.

$(2 S, 3 R, 4 S)-2$-(p-tolylamino)- $N$-benzyl-2,3,4,5-tetrahydro-2-methyl-5-oxo-4-phenylpyrano[3,2 -c]chromene-3-carboxamide, $6\{1,3,1,2\}$ :

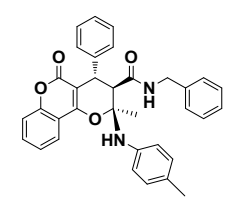

White powder (yield 81\%); m.p. 150.5-152.2 ${ }^{\circ} \mathrm{C} ;{ }^{1} \mathrm{H}$ NMR (400 MHz, DMSO) $\delta$ $8.74(\mathrm{~s}, \mathrm{NH}, 1 \mathrm{H}), 8.05(\mathrm{~d}, J=7.8 \mathrm{~Hz}$, aromatic, $1 \mathrm{H}), 7.64(\mathrm{t}, J=7.8 \mathrm{~Hz}$, aromatic, $1 \mathrm{H}), 7.45(\mathrm{t}, J=$ $7.5 \mathrm{~Hz}$, aromatic, $1 \mathrm{H}), 7.38(\mathrm{~d}, J=8.3 \mathrm{~Hz}$, aromatic, $1 \mathrm{H}), 7.28(\mathrm{~d}, J=4.6 \mathrm{~Hz}$, aromatic, 3H), 7.19 (s, aromatic, 5H), $6.94(\mathrm{~d}, J=7.9 \mathrm{~Hz}$, aromatic, $2 \mathrm{H}), 6.82(\mathrm{~d}, J=7.6 \mathrm{~Hz}$, aromatic, 4H), 6.02 (s, NH, 1H), 4.45 (dd, $J=15.1,6.9 \mathrm{~Hz}, \mathrm{CH}_{2}, 1 \mathrm{H}$ ), 4.37 (d, $J=11.4 \mathrm{~Hz}, \mathrm{CH}, 1 \mathrm{H}$ ), 4.03 (dd, $J=15.1$, $\left.3.7 \mathrm{~Hz}, \mathrm{CH}_{2}, 1 \mathrm{H}\right), 3.15(\mathrm{~d}, J=11.5 \mathrm{~Hz}, \mathrm{CH}, 1 \mathrm{H}), 2.14\left(\mathrm{~s}, \mathrm{CH}_{3}, 3 \mathrm{H}\right), 1.75\left(\mathrm{~s}, \mathrm{CH}_{3}, 3 \mathrm{H}\right) ;{ }^{13} \mathrm{C} \mathrm{NMR}$ (101 MHz, DMSO) $\delta$ 170.13, 160.12, 157.59, 152.73, 141.73, 141.50, 138.62, 132.49, 129.82, $128.72,128.56,128.14,127.44,127.22,126.91,124.78,123.10,118.12,116.75,115.63,105.27$, 90.12, 56.91, 42.52, 40.59, 40.38, 40.17, 39.96, 39.75, 39.54, 39.33, 22.85, 20.47; HRMS: calcd for $\mathrm{C}_{34} \mathrm{H}_{30} \mathrm{~N}_{2} \mathrm{O}_{4} 530.2206$, found $531.2267[\mathrm{M}+\mathrm{H}]$.

(2S,3R,4S)- $N$-benzyl-4-(4-methoxyphenyl)-2-methyl-5-oxo-2-(p-tolylamino)-2,3,4,5-tetrahydr opyrano[3,2-c] chromene-3-carboxamide, $6\{1,3,1,4\}$ :

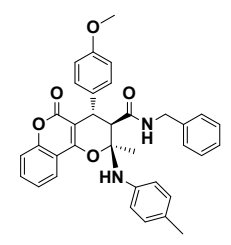

White powder (yield 38\%); m.p. 140.2-142.4 ${ }^{\circ} \mathrm{C} ;{ }^{1} \mathrm{H}$ NMR (400 MHz, $\left.\mathrm{CDCl}_{3}\right) \delta$ $7.97(\mathrm{~d}, J=7.8 \mathrm{~Hz}$, aromatic, $1 \mathrm{H}), 7.49$ (t, $J=7.7 \mathrm{~Hz}$, aromatic, $1 \mathrm{H}), 7.33$ (t, $J=7.5 \mathrm{~Hz}$, aromatic, 1H), 7.23 (s, aromatic, 4H), $7.11(\mathrm{~d}, J=7.1 \mathrm{~Hz}$, aromatic, $2 \mathrm{H}), 6.97(\mathrm{~d}, J=8.0 \mathrm{~Hz}$, aromatic, $2 \mathrm{H})$, $6.86(\mathrm{~d}, J=8.0 \mathrm{~Hz}$, aromatic, $4 \mathrm{H}), 6.77(\mathrm{~d}, J=7.7 \mathrm{~Hz}$, aromatic, $2 \mathrm{H}), 5.96(\mathrm{~s}, \mathrm{NH}, 1 \mathrm{H}), 5.66(\mathrm{~s}$, $\mathrm{NH}, 1 \mathrm{H}), 4.48-4.37\left(\mathrm{~m}, \mathrm{CH}+\mathrm{CH}_{2}, 2 \mathrm{H}\right), 4.18\left(\mathrm{~d}, J=14.5 \mathrm{~Hz}, \mathrm{CH}_{2}, 1 \mathrm{H}\right), 3.76\left(\mathrm{~s}, \mathrm{CH}_{3}, 3 \mathrm{H}\right), 2.70$ (d, $J=10.7 \mathrm{~Hz}, \mathrm{CH}, 1 \mathrm{H}), 2.22\left(\mathrm{~s}, \mathrm{CH}_{3}, 3 \mathrm{H}\right), 1.77\left(\mathrm{~s}, \mathrm{CH}_{3}, 3 \mathrm{H}\right) ;{ }^{13} \mathrm{C} \mathrm{NMR}\left(100 \mathrm{MHz}, \mathrm{CDCl}_{3}\right) \delta$ 170.63, 160.58, 158.56, 157.60, 153.00, 141.18, 136.89, 134.74, 133.01, 131.47, 129.53, 128.56, 127.56, 123.78, 122.54, 118.47, 116.66, 115.81, 114.24, 105.23, 89.73,59.44, 55.12, 43.66, 39.40, 22.83, 20.43; HRMS: calcd for $\mathrm{C}_{35} \mathrm{H}_{32} \mathrm{~N}_{2} \mathrm{O}_{5} 560.2311$, found $561.2370[\mathrm{M}+\mathrm{H}]$.

(2S,3R,4S)- $N$-benzyl-4-(4-chlorophenyl)-2-((4-chlorophenyl)amino)-2-methyl-5-oxo-2,3,4,5-te trahydropyrano[3,2-c]chromene-3-carboxamide, $6\{1,3,3,1\}$ : 


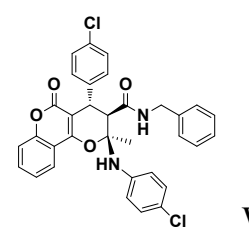

White powder (yield 70\%); m.p. 148.5-150.5 ${ }^{\circ}$; ${ }^{1} \mathrm{H}$ NMR (400 MHz, $\left.\mathrm{CDCl}_{3}\right) \delta$ $7.95(\mathrm{~d}, J=7.9 \mathrm{~Hz}$, aromatic, $1 \mathrm{H}), 7.53(\mathrm{t}, J=7.8 \mathrm{~Hz}$, aromatic, $1 \mathrm{H}), 7.35(\mathrm{t}, J=7.6 \mathrm{~Hz}$, aromatic, 1H), 7.25 (d, $J=9.2 \mathrm{~Hz}$, aromatic, 4H), $7.18(\mathrm{~d}, J=7.9 \mathrm{~Hz}$, aromatic, $2 \mathrm{H}), 7.11$ (dd, $J=11.3,8.5$ $\mathrm{Hz}$, aromatic, 4H), $6.88(\mathrm{t}, J=8.0 \mathrm{~Hz}$, aromatic, 4H), $6.11(\mathrm{~s}, \mathrm{NH}, 1 \mathrm{H}), 5.73(\mathrm{~s}, \mathrm{NH}, 1 \mathrm{H}), 4.56-$ $4.33\left(\mathrm{~m}, \mathrm{CH}+\mathrm{CH}_{2}, 2 \mathrm{H}\right), 4.13\left(\mathrm{dd}, J=14.5,5.2 \mathrm{~Hz}, \mathrm{CH}_{2}, 1 \mathrm{H}\right), 2.65(\mathrm{~d}, J=11.5 \mathrm{~Hz}, \mathrm{CH}, 1 \mathrm{H}), 1.77$ (s, $\left.\mathrm{CH}_{3}, 3 \mathrm{H}\right) ;{ }^{13} \mathrm{C}$ NMR $\left(101 \mathrm{MHz}, \mathrm{CDCl}_{3}\right) \delta 170.26,162.89,162.48,161.63,141.28,139.79$, $136.78,132.56,129.57,128.85,128.64,127.64,127.44,118.02,101.41,100.26,89.12,58.85$, 43.57, 38.93, 22.65, 20.47, 19.80; HRMS: calcd for $\mathrm{C}_{33} \mathrm{H}_{26} \mathrm{Cl}_{2} \mathrm{~N}_{2} \mathrm{O}_{4} 584.1270$, found 585.1329 $[\mathrm{M}+\mathrm{H}]$.

$(2 S, 3 R, 4 S)$-4-(4-chlorophenyl)-2-methyl-5-oxo- $N$-(p-tolyl)-2-(p-tolylamino)-2,3,4,5-tetrahydro pyrano[3,2-c]chromene-3-carboxamide, $6\{5,3,1,1\}$ :

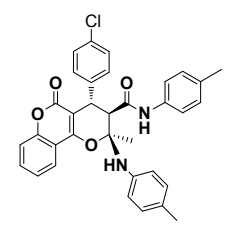

White powder (yield 85\%); m.p. 172.9-176.8 ${ }^{\circ}$; ${ }^{1} \mathrm{H}$ NMR (400 MHz, $\left.\mathrm{CDCl}_{3}\right) \delta$ $8.01(\mathrm{~d}, J=7.7 \mathrm{~Hz}$, aromatic, $1 \mathrm{H}), 7.50(\mathrm{t}, J=7.7 \mathrm{~Hz}$, aromatic, $1 \mathrm{H}), 7.36(\mathrm{t}, J=7.4 \mathrm{~Hz}$, aromatic, $1 \mathrm{H}), 7.17(\mathrm{~d}, J=7.9 \mathrm{~Hz}$, aromatic, $3 \mathrm{H}), 7.10(\mathrm{~d}, J=12.9 \mathrm{~Hz}$, aromatic, $6 \mathrm{H}), 6.97(\mathrm{~d}, J=7.4 \mathrm{~Hz}$, aromatic, 3H), $6.86(\mathrm{~d}, J=7.7 \mathrm{~Hz}$, aromatic, $2 \mathrm{H}), 5.92(\mathrm{~s}, \mathrm{NH}, 1 \mathrm{H}), 4.48(\mathrm{~d}, J=11.4 \mathrm{~Hz}, \mathrm{CH}, 1 \mathrm{H})$, $2.82(\mathrm{~d}, J=11.4 \mathrm{~Hz}, \mathrm{CH}, 1 \mathrm{H}), 2.31\left(\mathrm{~s}, \mathrm{CH}_{3}, 3 \mathrm{H}\right), 2.21\left(\mathrm{~s}, \mathrm{CH}_{3}, 3 \mathrm{H}\right), 1.83\left(\mathrm{~s}, \mathrm{CH}_{3}, 3 \mathrm{H}\right) ;{ }^{13} \mathrm{C} \mathrm{NMR}$ $\left(101 \mathrm{MHz}, \mathrm{CDCl}_{3}\right) \delta 168.88,162.47,160.76,153.00,140.86,139.81,135.34,133.59,132.77$, $131.81,129.96,129.56,129.47,128.96,128.49,128.46,124.04,122.64,121.07,118.68,116.73$, $115.68,104.45,90.07,59.43,53.52,39.75,22.76,20.84,20.43$; HRMS: calcd for $\mathrm{C}_{34} \mathrm{H}_{29} \mathrm{ClN}_{2} \mathrm{O}_{4}$ 564.1816 , found $565.1876[\mathrm{M}+\mathrm{H}]$.

(2S,3R,4S)-2-(p-tolylamino)-4-(4-chlorophenyl)-2,3,4,5-tetrahydro-2-methyl-5-oxo- $N$-phenyl pyrano[3,2-c] chromene-3-carboxamide, $6\{7,3,1,1\}$ :

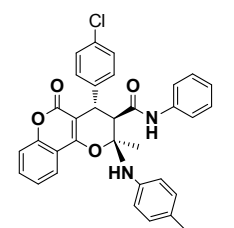

White powder (yield 91\%); m.p. 191.8-193.6 ${ }^{\circ}$; ${ }^{1} \mathrm{H}$ NMR (400 MHz, DMSO) $\delta$ 10.19 (s, NH, 1H), 8.08 (d, $J=7.7$, aromatic, $1 \mathrm{H}), 7.67$ (t, $J=7.5 \mathrm{~Hz}$, aromatic, $1 \mathrm{H}), 7.48$ (d, $J=7.6$ $\mathrm{Hz}$, aromatic, $1 \mathrm{H}), 7.42(\mathrm{t}, J=8.9 \mathrm{~Hz}$, aromatic, $3 \mathrm{H}), 7.31(\mathrm{~d}, J=8.3 \mathrm{~Hz}$, aromatic, 4H), $7.24(\mathrm{~d}$, $J=8.1 \mathrm{~Hz}$, aromatic, $2 \mathrm{H}), 7.10(\mathrm{t}, J=7.2 \mathrm{~Hz}$, aromatic, $1 \mathrm{H}), 7.02-6.81$ (m, aromatic, $4 \mathrm{H}), 6.02(\mathrm{~s}$, NH, 1H), 4.50 (d, $J=11.4 \mathrm{~Hz}, \mathrm{CH}, 1 \mathrm{H}), 3.24$ (d, $J=11.4 \mathrm{~Hz}, \mathrm{CH}, 1 \mathrm{H}), 2.14\left(\mathrm{~s}, \mathrm{CH}_{3}, 3 \mathrm{H}\right), 1.83$ (s, $\left.\mathrm{CH}_{3}, 3 \mathrm{H}\right) ;{ }^{13} \mathrm{C}$ NMR $(101 \mathrm{MHz}$, DMSO) $\delta 168.88,160.18,158.08,152.79,141.16,140.83,138.04$, 
$132.64,131.47,129.79,129.69,129.25,129.00,128.70,124.85,123.20,120.47,118.44,116.81$, 115.64, 104.65, 90.28, 57.57, 38.93, 22.89, 20.48; HRMS: calcd for $\mathrm{C}_{33} \mathrm{H}_{27} \mathrm{ClN}_{2} \mathrm{O}_{4}$ 550.1659, found 549.1587 [M-H].

(2S,3R,4S)-N-benzyl-4-(4-chlorophenyl)-2,7-dimethyl-5-oxo-2-(p-tolylamino)-2,3,4,5-tetrahyd ropyrano[4,3-b]pyran-3-carboxamide, $6\{1,4,1,1\}$ :

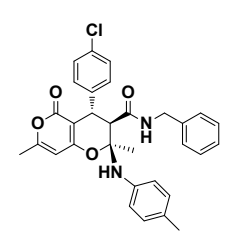

White powder (yield 78\%); m.p. 180.5-182.1 ${ }^{\circ} \mathrm{C} ;{ }^{1} \mathrm{H}$ NMR (400 MHz, $\mathrm{CDCl}_{3}$ ) $\delta$ $7.24(\mathrm{~d}, J=1.7 \mathrm{~Hz}$, aromatic, 3H), $7.17(\mathrm{~d}, J=8.4 \mathrm{~Hz}$, aromatic, 2H), $7.07(\mathrm{~d}, J=8.3 \mathrm{~Hz}$, aromatic, $2 \mathrm{H}), 7.01(\mathrm{~d}, J=8.1 \mathrm{~Hz}$, aromatic, $2 \mathrm{H}), 6.83(\mathrm{~d}, J=8.2 \mathrm{~Hz}$, aromatic, $4 \mathrm{H}), 5.90$ (s, aromatic, $1 \mathrm{H}), 5.82(\mathrm{~s}, 2 \mathrm{NH}, 2 \mathrm{H}), 4.43\left(\mathrm{dd}, J=14.8,6.7 \mathrm{~Hz}, \mathrm{CH}_{2}, 1 \mathrm{H}\right), 4.27(\mathrm{~d}, J=11.5 \mathrm{~Hz}, \mathrm{CH}$, 1H), 4.08 (dd, $\left.J=14.8,4.5 \mathrm{~Hz}, \mathrm{CH}_{2}, 1 \mathrm{H}\right), 2.53$ (d, $\left.J=11.5 \mathrm{~Hz}, \mathrm{CH}_{2}, 1 \mathrm{H}\right), 2.25$ (s, $\left.\mathrm{CH}_{3}, 3 \mathrm{H}\right), 2.14$ (s, $\left.\mathrm{CH}_{3}, 3 \mathrm{H}\right), 1.62$ (s, $\left.\mathrm{CH}_{3}, 3 \mathrm{H}\right) ;{ }^{13} \mathrm{C}$ NMR $\left(101 \mathrm{MHz}, \mathrm{CDCl}_{3}\right) \delta$ 170.26, 162.89, 162.48, 161.63, $141.28,139.79,136.78,132.56,129.57,128.85,128.64,127.64,127.44,118.02,101.41,100.26$, 89.12, 58.85, 43.57, 38.93, 22.65, 20.47, 19.80; HRMS: calcd for $\mathrm{C}_{31} \mathrm{H}_{29} \mathrm{ClN}_{2} \mathrm{O}_{4} 528.1816$, found $529.1883[\mathrm{M}+\mathrm{H}]$.

(2S,3R,4S)- $N$-benzyl-2,7-dimethyl-5-oxo-4-phenyl-2-(p-tolylamino)-2,3,4,5-tetrahydropyrano [4,3-b]pyran-3-carboxamide, $6\{1,4,1,2\}$ :

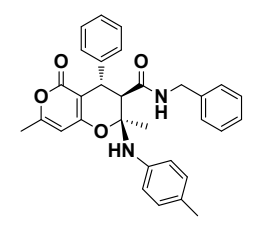

White powder (yield 71\%); m.p. 178.6-181.5 ${ }^{\circ} \mathrm{C} ;{ }^{1} \mathrm{H} \mathrm{NMR}\left(400 \mathrm{MHz}, \mathrm{CDCl}_{3}\right) \delta$ $7.22(\mathrm{~d}, J=7.3 \mathrm{~Hz}$, aromatic, $6 \mathrm{H}), 7.15(\mathrm{~d}, J=6.7 \mathrm{~Hz}$, aromatic, $2 \mathrm{H}), 7.01(\mathrm{~d}, J=7.9 \mathrm{~Hz}$, aromatic, $2 \mathrm{H}), 6.84(\mathrm{t}, J=6.8 \mathrm{~Hz}$, aromatic, $4 \mathrm{H}), 5.89(\mathrm{~s}$, aromatic $\mathrm{H}+\mathrm{NH}, 2 \mathrm{H}), 5.68(\mathrm{~s}, \mathrm{NH}, 1 \mathrm{H})$, $4.36-4.24\left(\mathrm{~m}, \mathrm{CH}+\mathrm{CH}_{2}, 2 \mathrm{H}\right), 4.13\left(\mathrm{dd}, J=14.8,4.7 \mathrm{~Hz}, \mathrm{CH}_{2}, 1 \mathrm{H}\right), 2.58(\mathrm{~d}, J=11.5 \mathrm{~Hz}, \mathrm{CH}$, $1 \mathrm{H}), 2.25\left(\mathrm{~s}, \mathrm{CH}_{3}, 3 \mathrm{H}\right), 2.14\left(\mathrm{~s}, \mathrm{CH}_{3}, 3 \mathrm{H}\right), 1.62\left(\mathrm{~s}, \mathrm{CH}_{3}, 3 \mathrm{H}\right) ;{ }^{13} \mathrm{C} \mathrm{NMR}\left(101 \mathrm{MHz}, \mathrm{CDCl}_{3}\right) \delta$ 170.63, 162.66, 162.49, 161.34, 141.47, 141.26, 136.87, 129.55, 129.37, 128.70, 128.54, 127.48, 127.33, 126.85, 117.98, 101.98, 100.22, 89.07, 59.12, 43.59, 39.51, 22.63, 20.47, 19.79; HRMS: calcd for $\mathrm{C}_{31} \mathrm{H}_{30} \mathrm{ClN}_{2} \mathrm{O}_{4} 494.2206$, found $495.2266[\mathrm{M}+\mathrm{H}]$.

(2S,3R,4S)-N-benzyl-4-(4-methoxyphenyl)-2,7-dimethyl-5-oxo-2-(p-tolylamino)-2,3,4,5-tetrah ydropyrano[4,3-b]pyran-3-carboxamide, $6\{, 1,4,1,4\}$ : 


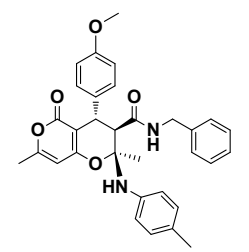

White powder (yield 75\%); m.p. 157.2-162.2 ${ }^{\circ} \mathrm{C} ;{ }^{1} \mathrm{H} \mathrm{NMR}\left(400 \mathrm{MHz}, \mathrm{CDCl}_{3}\right) \delta$ 7.22 (s, aromatic, 3H), 7.09 (d, $J=7.9 \mathrm{~Hz}$, aromatic, 2H), 7.01 (d, $J=8.0 \mathrm{~Hz}$, aromatic, 2H), 6.84 $(\mathrm{d}, J=8.0 \mathrm{~Hz}$, aromatic, $4 \mathrm{H}), 6.77(\mathrm{~d}, J=8.1 \mathrm{~Hz}$, aromatic, $2 \mathrm{H}), 5.87$ (s, aromatic $\mathrm{H}+\mathrm{NH}, 2 \mathrm{H})$, $5.58(\mathrm{~d}, J=20.9 \mathrm{~Hz}, \mathrm{NH}, 1 \mathrm{H}), 4.39$ (dd, $\left.J=14.7,6.3 \mathrm{~Hz}, \mathrm{CH}_{2}, 1 \mathrm{H}\right), 4.25(\mathrm{~d}, J=11.5 \mathrm{~Hz}, \mathrm{CH}, 1 \mathrm{H})$, 4.14 (d, $\left.J=14.6 \mathrm{~Hz}, \mathrm{CH}_{2}, 1 \mathrm{H}\right), 3.77\left(\mathrm{~s}, \mathrm{CH}_{3}, 3 \mathrm{H}\right), 2.56$ (d, $\left.J=11.5 \mathrm{~Hz}, \mathrm{CH}, 1 \mathrm{H}\right), 2.25\left(\mathrm{~s}, \mathrm{CH}_{3}\right.$, $3 \mathrm{H}), 2.15$ (s, $\left.\mathrm{CH}_{3}, 3 \mathrm{H}\right), 1.63$ (s, $\left.\mathrm{CH}_{3}, 3 \mathrm{H}\right) ;{ }^{13} \mathrm{C} \mathrm{NMR}\left(101 \mathrm{MHz}, \mathrm{CDCl}_{3}\right) \delta 170.75,162.50,161.26$, 158.44, 141.49, 136.99, 133.07, 129.53, 129.33, 128.50, 127.49, 117.97, 114.13, 102.16, 100.24, 89.11, 59.17, 55.10, 43.53, 38.71, 22.65, 20.47, 19.77; HRMS: calcd for $\mathrm{C}_{32} \mathrm{H}_{32} \mathrm{~N}_{2} \mathrm{O}_{5}$ 524.2311, found $525.2371[\mathrm{M}+\mathrm{H}]$.

(2S,3R,4S)- $N$-benzyl-4-(4-chlorophenyl)-2-((4-chlorophenyl)amino)-2,7-dimethyl-5-oxo-2,3,4, 5-tetrahydropyrano[4,3-b]pyran-3-carboxamide, $6\{1,4,3,1\}$ :

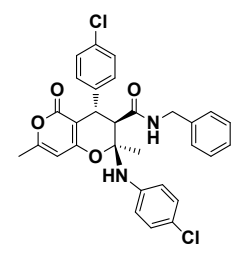

White powder (yield 75\%); m.p. 188.8-191.6 ${ }^{\circ}$; ${ }^{1} \mathrm{H}$ NMR (400 MHz, DMSO) $\delta$ $8.70(\mathrm{dd}, J=6.7,4.5 \mathrm{~Hz}, \mathrm{NH}, 1 \mathrm{H}), 7.31(\mathrm{~d}, J=8.3 \mathrm{~Hz}$, aromatic, $2 \mathrm{H}), 7.20(\mathrm{~d}, J=8.4 \mathrm{~Hz}$, aromatic, $5 \mathrm{H}), 7.13(\mathrm{~d}, J=8.3 \mathrm{~Hz}$, aromatic, $2 \mathrm{H}), 6.91(\mathrm{~d}, J=8.7 \mathrm{~Hz}$, aromatic, $2 \mathrm{H}), 6.84-6.68$ (m, aromatic, 2H), $6.19(\mathrm{~d}, J=13.5 \mathrm{~Hz}$, aromatic $\mathrm{H}+\mathrm{NH}, 2 \mathrm{H}), 4.46\left(\mathrm{dd}, J=15.2,7.2 \mathrm{~Hz}, \mathrm{CH}_{2}\right.$, 1H), 4.20 (d, $J=11.6 \mathrm{~Hz}, \mathrm{CH}, 1 \mathrm{H}), 3.98$ (dd, $\left.J=15.3,4.0 \mathrm{~Hz}, \mathrm{CH}_{2}, 1 \mathrm{H}\right), 2.97$ (d, $J=11.6 \mathrm{~Hz}, \mathrm{CH}$, $1 \mathrm{H}), 2.16\left(\mathrm{~s}, \mathrm{CH}_{3}, 3 \mathrm{H}\right), 1.60\left(\mathrm{~s}, \mathrm{CH}_{3}, 3 \mathrm{H}\right) ;{ }^{13} \mathrm{C} \mathrm{NMR}(101 \mathrm{MHz}, \mathrm{DMSO}) \delta 169.92,162.68,162.19$, $161.93,143.25,140.64,138.56,131.43,129.97,129.18,128.60,128.49,128.32,127.41,127.29$, 123.56, 120.62, 119.09, 101.36, 100.23, 88.82, 56.31, 42.43, 38.16, 22.57, 19.65; HRMS: calcd for $\mathrm{C}_{30} \mathrm{H}_{26} \mathrm{Cl}_{2} \mathrm{~N}_{2} \mathrm{O}_{4} 548.1270$, found $549.1332[\mathrm{M}+\mathrm{H}]$. 


\section{Copies of NMR Spectra:}

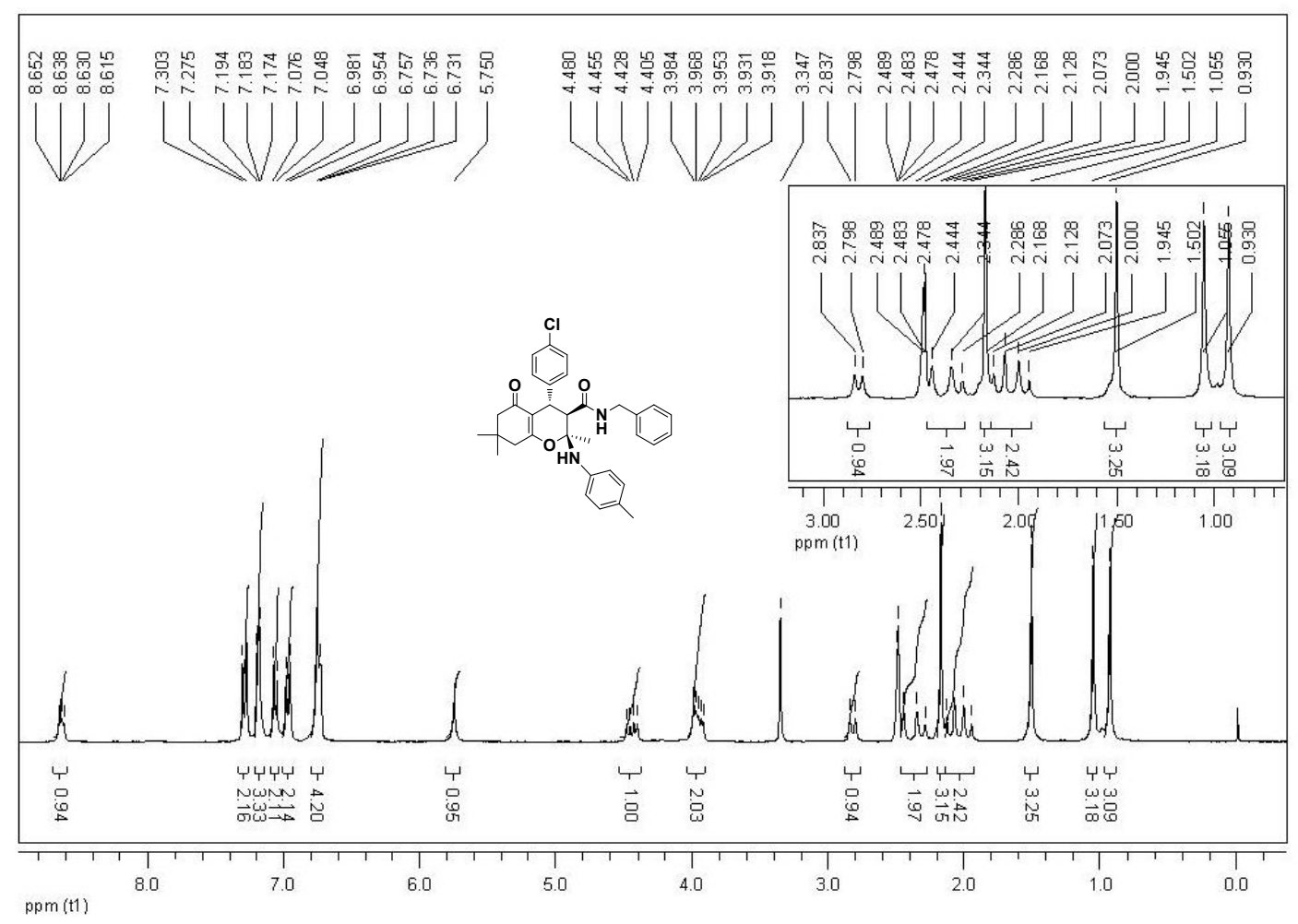

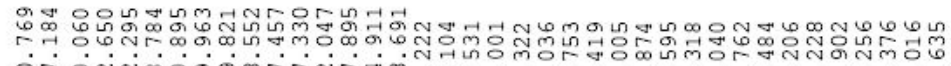

م
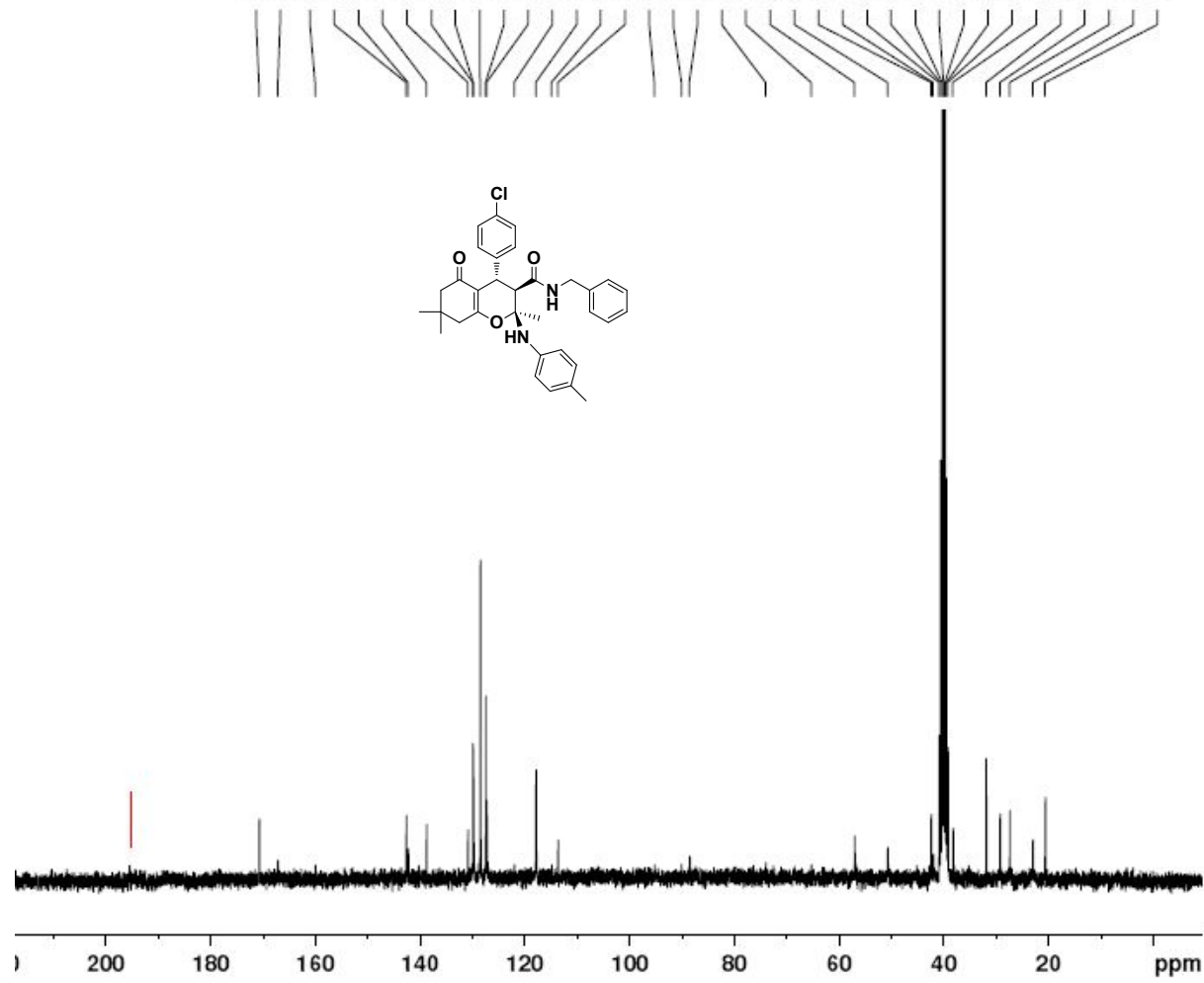


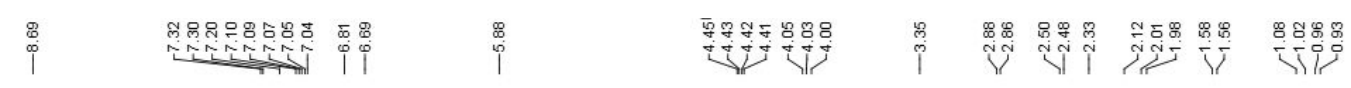

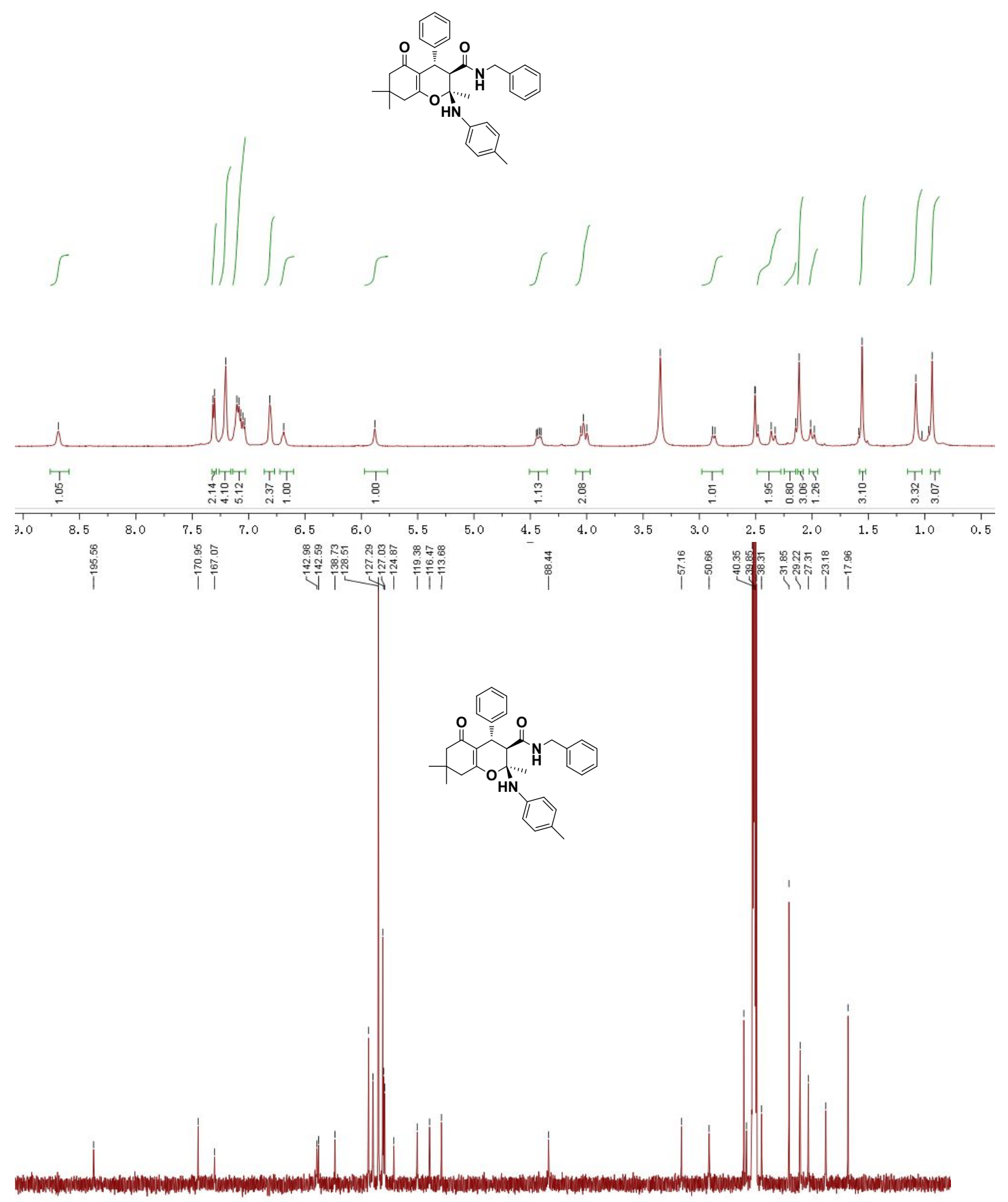

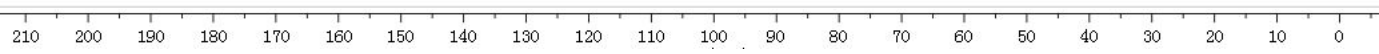




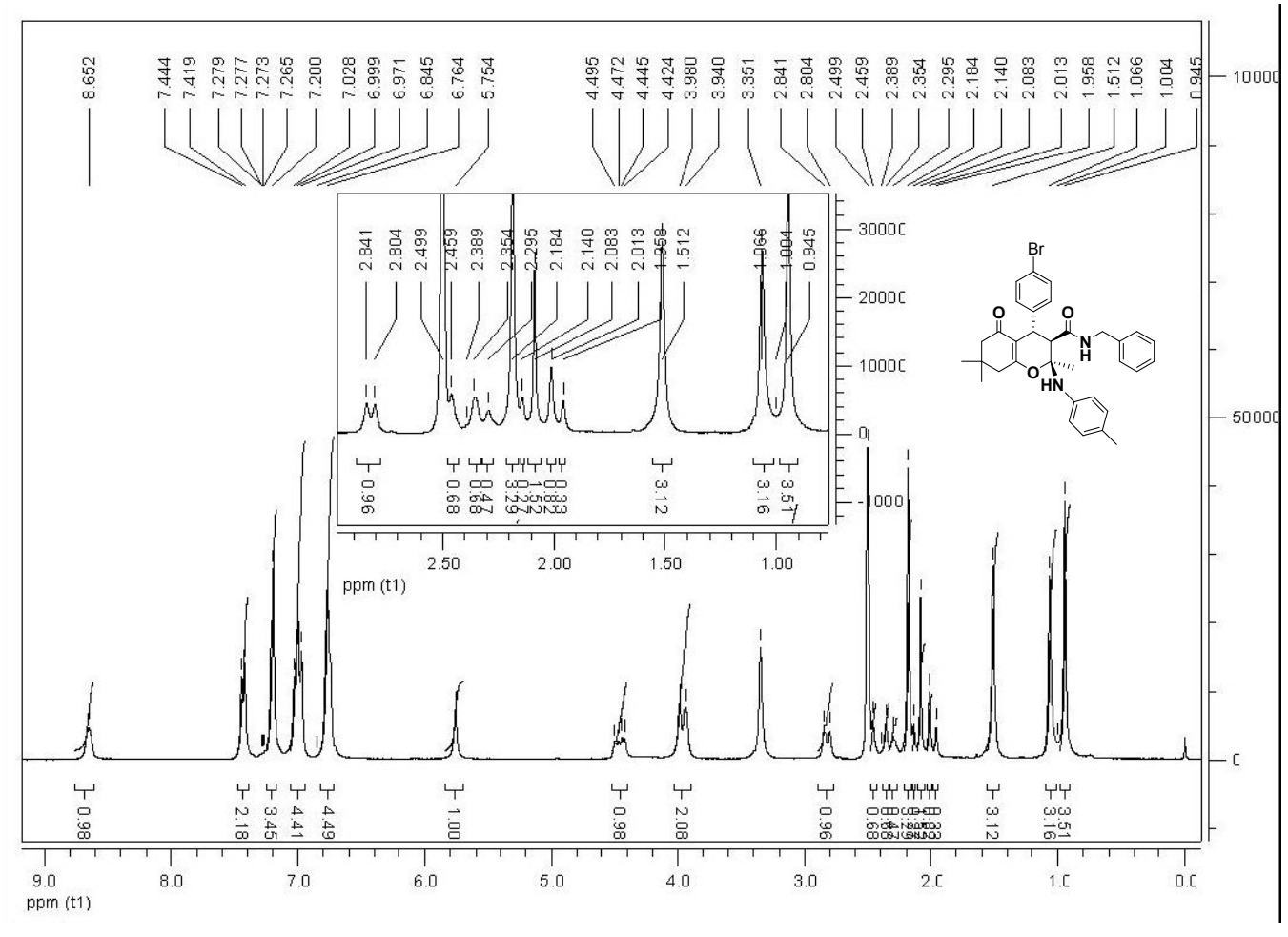

$\begin{array}{llll}1 & 1 \\ 0 & 1\end{array}$
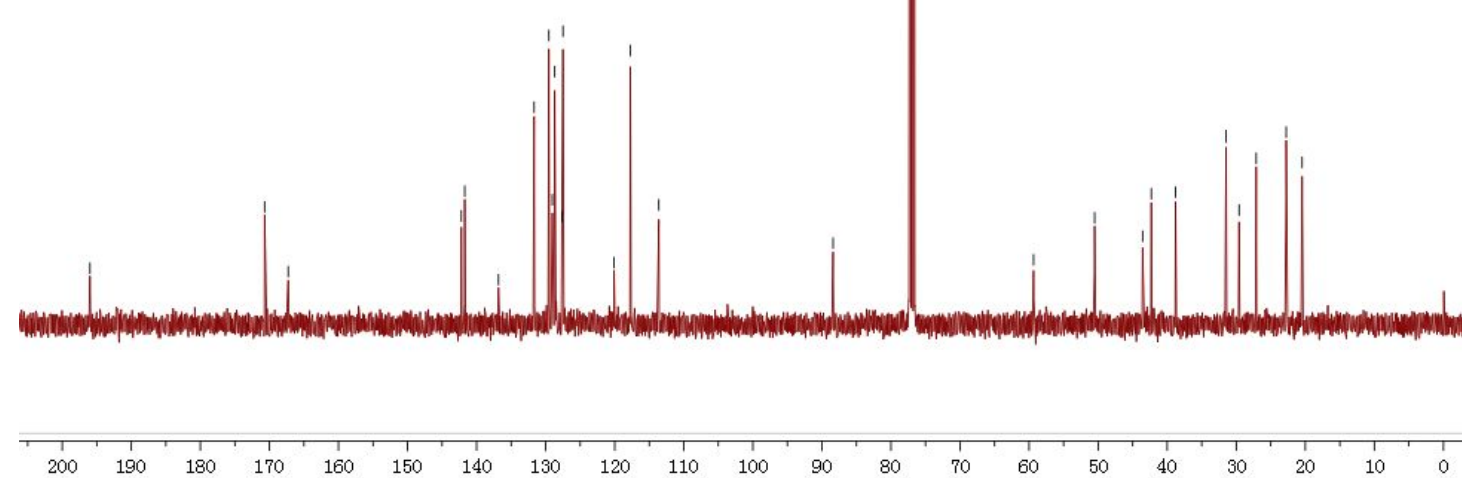

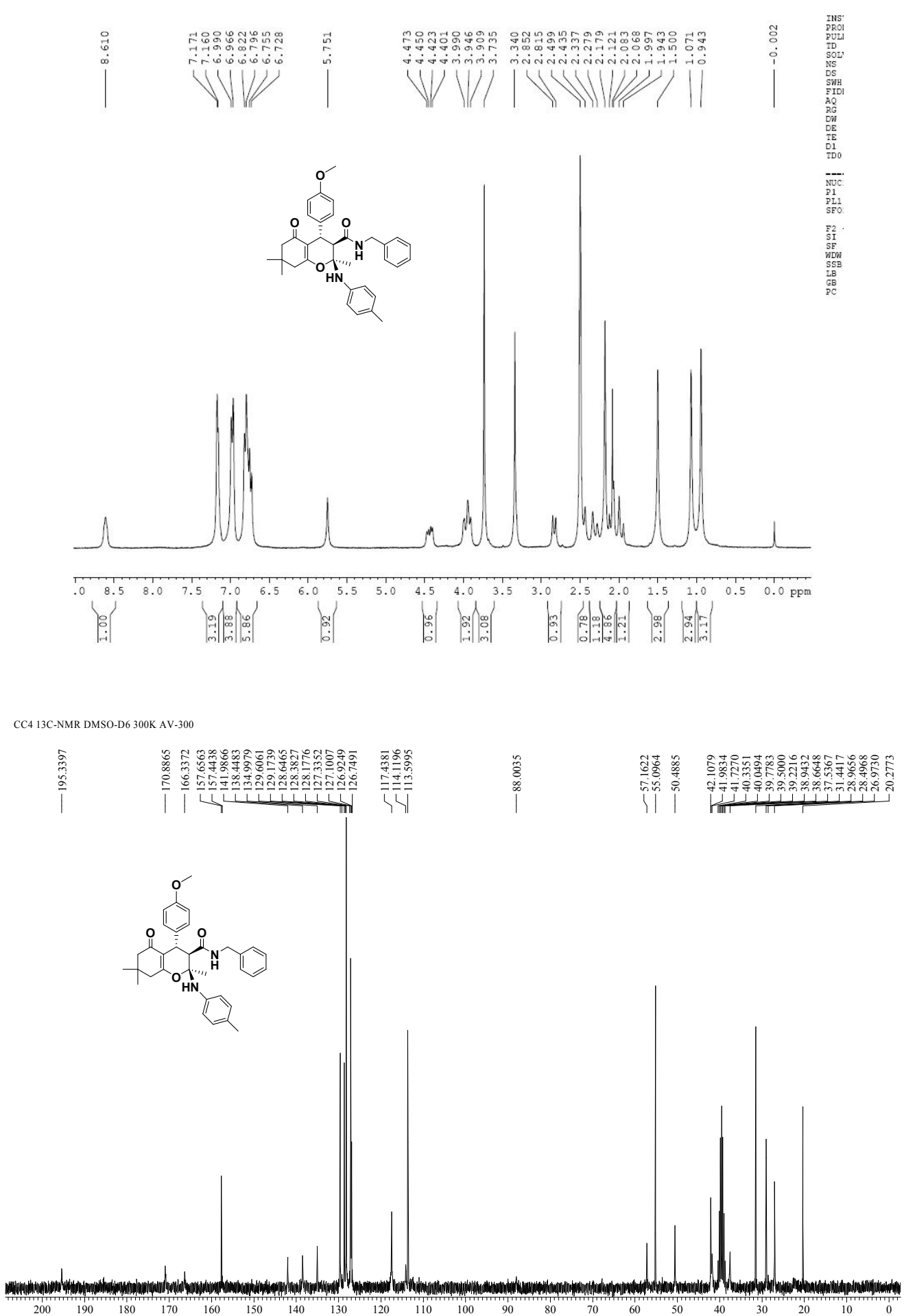


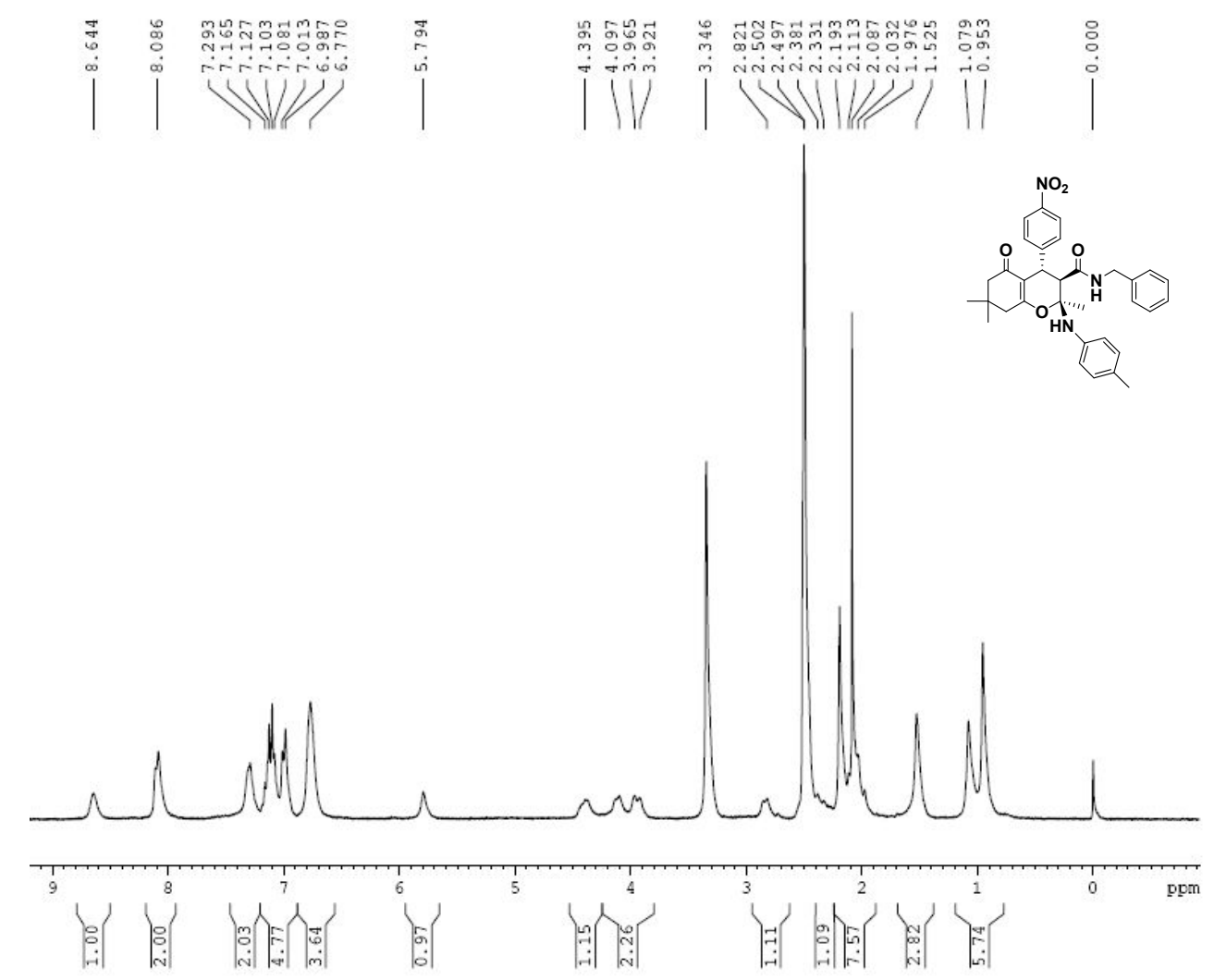

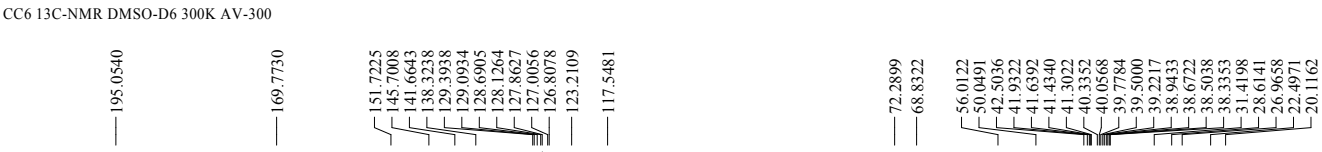

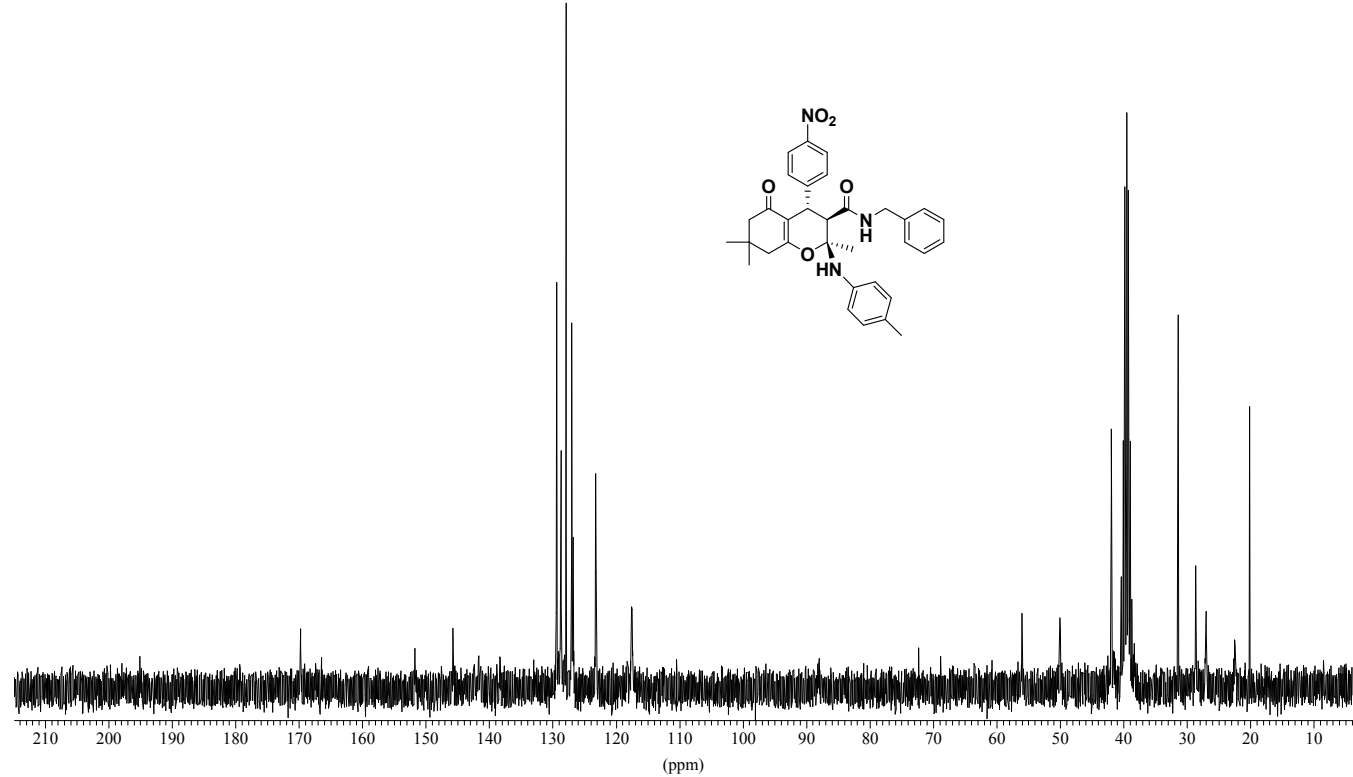




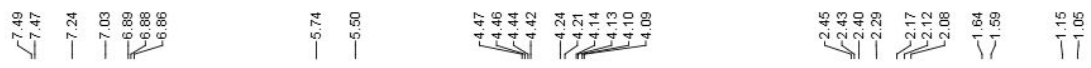
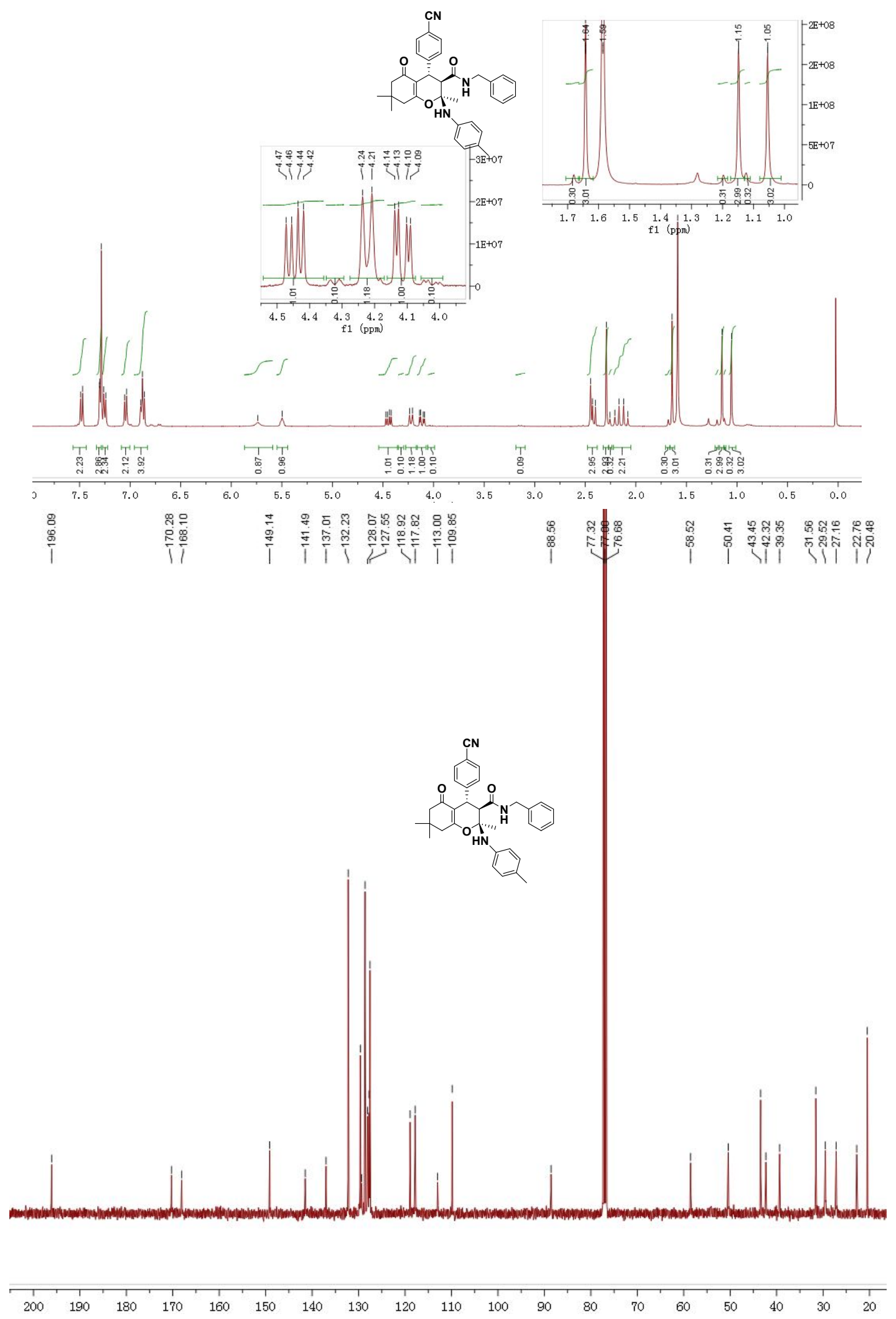

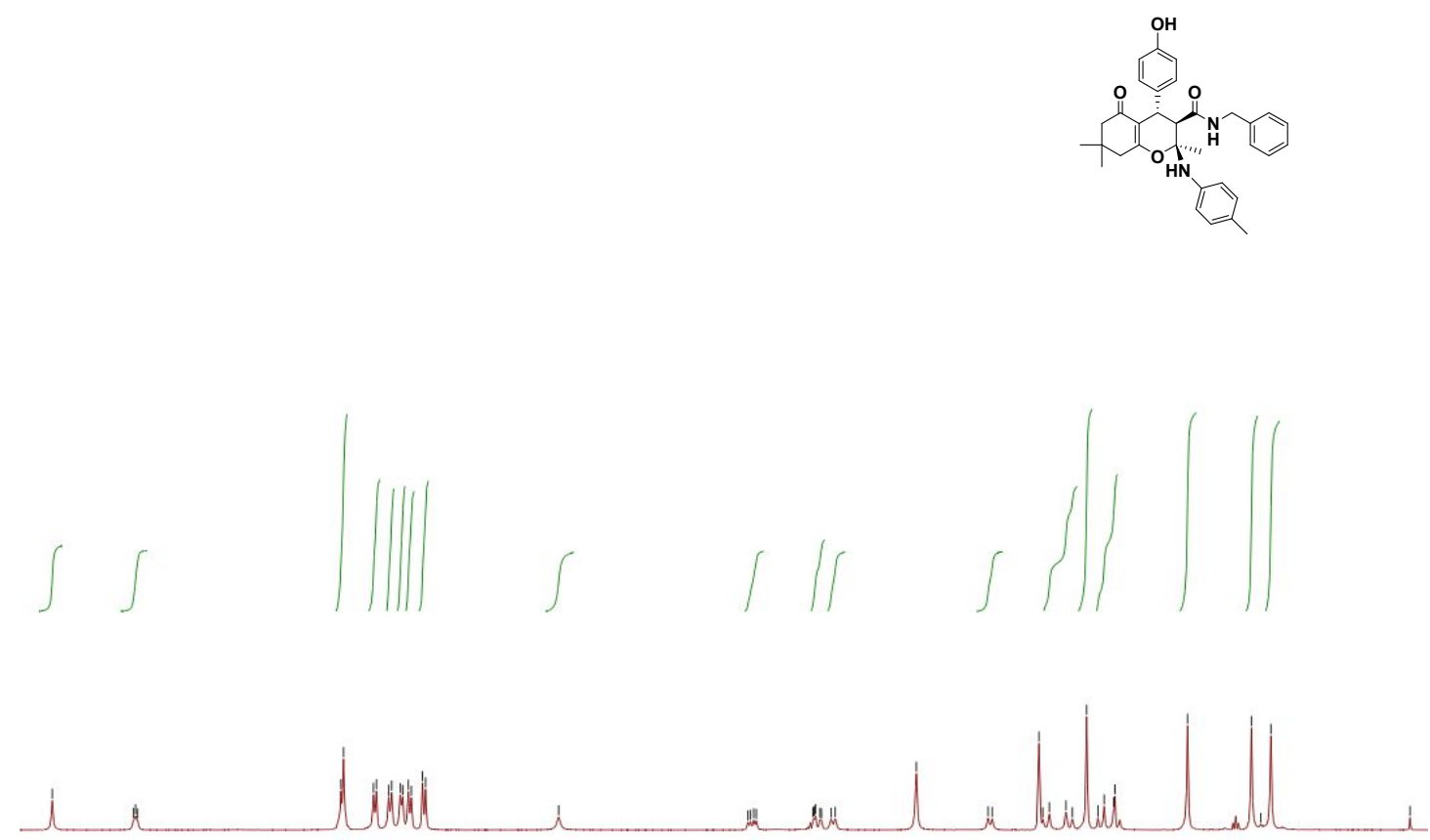

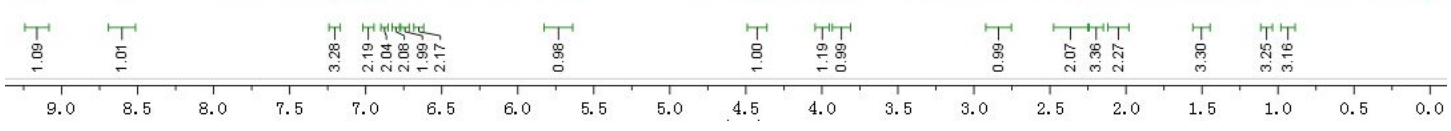

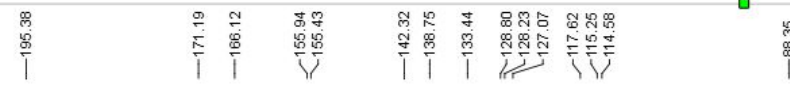
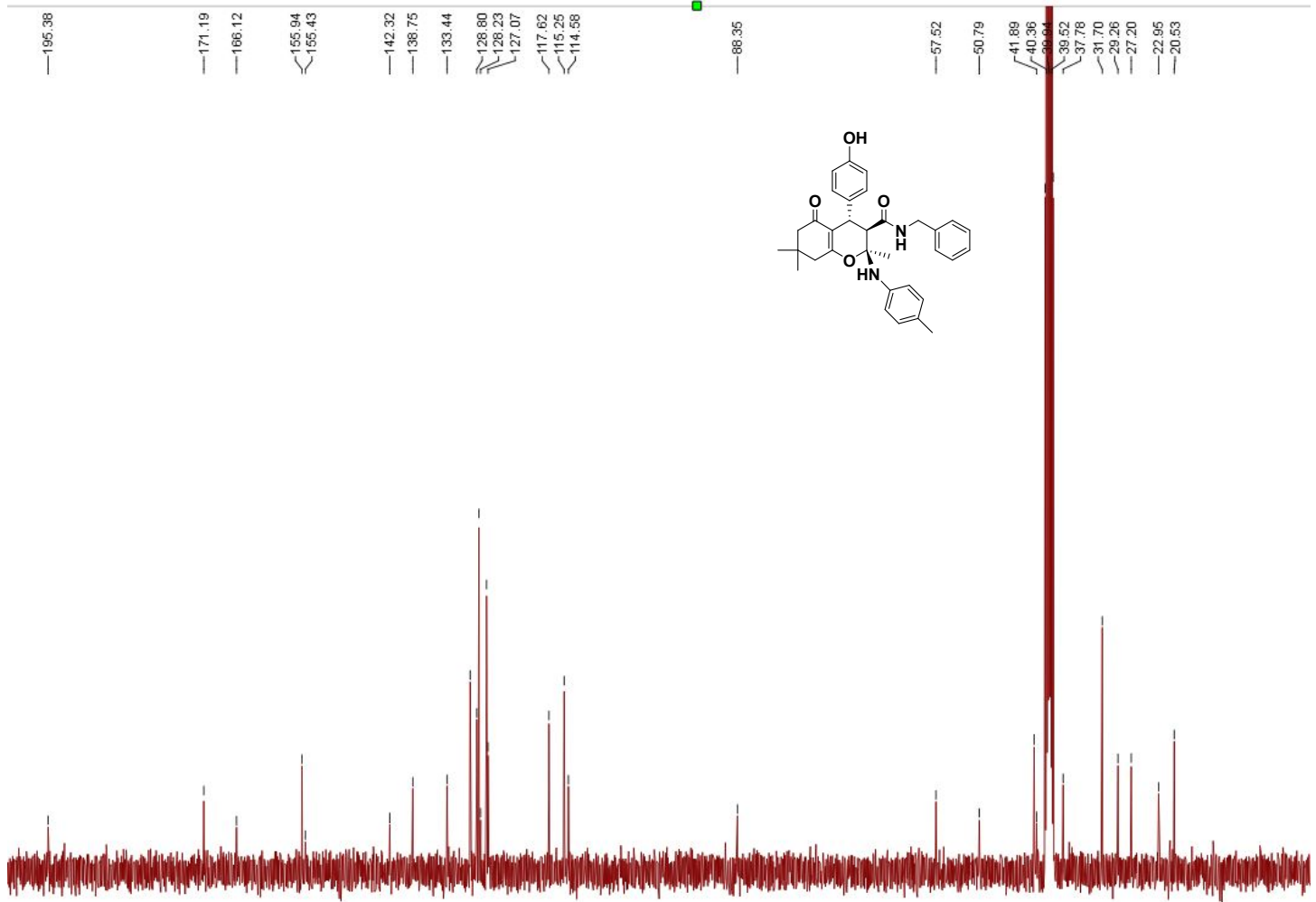

$200+190+1$

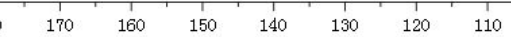

100 


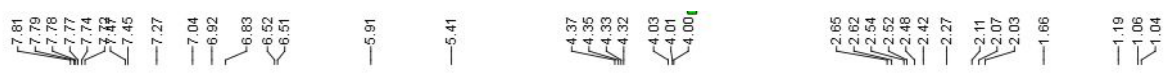
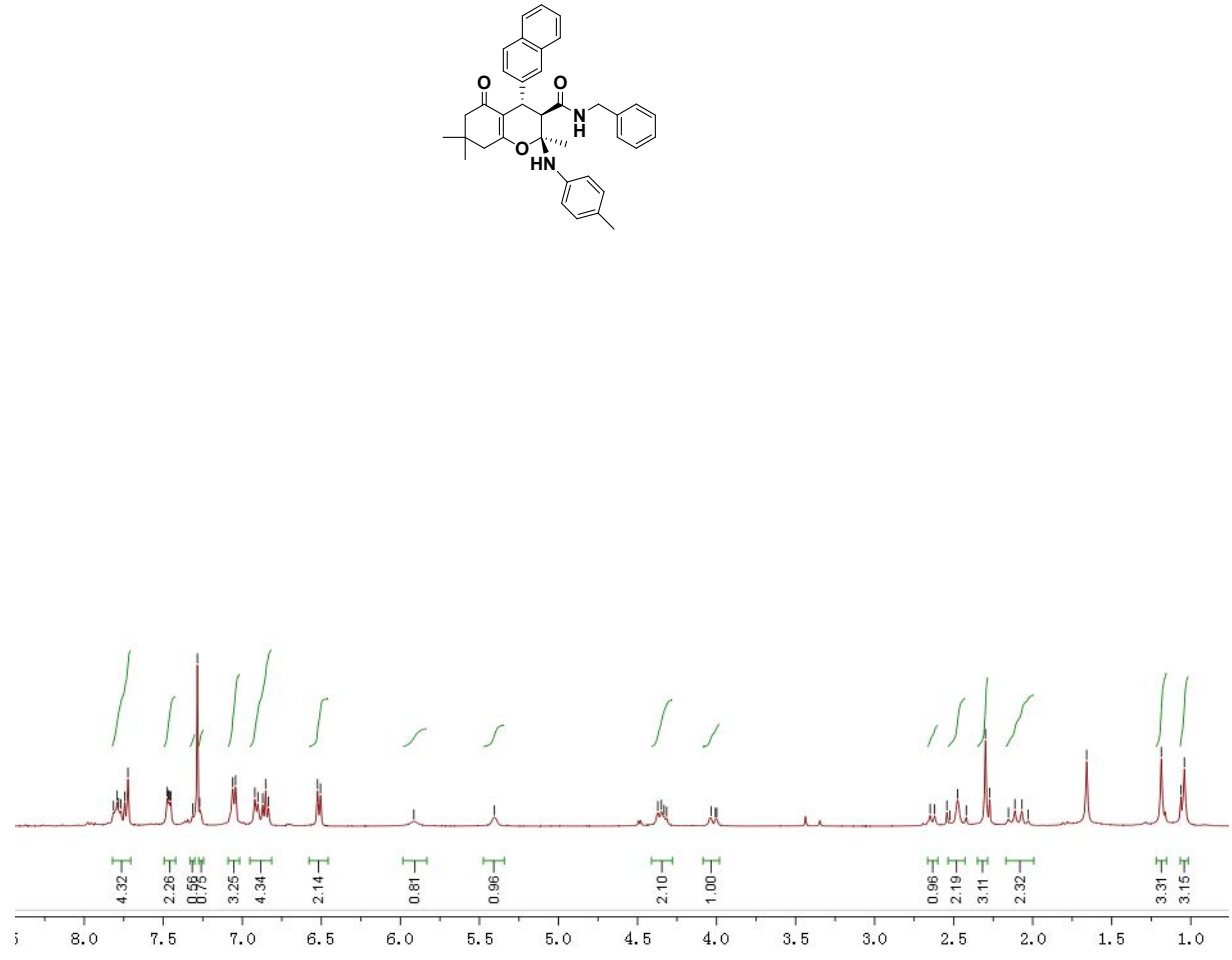

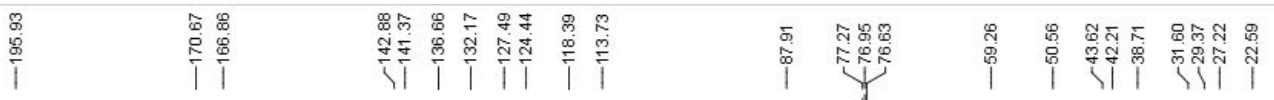
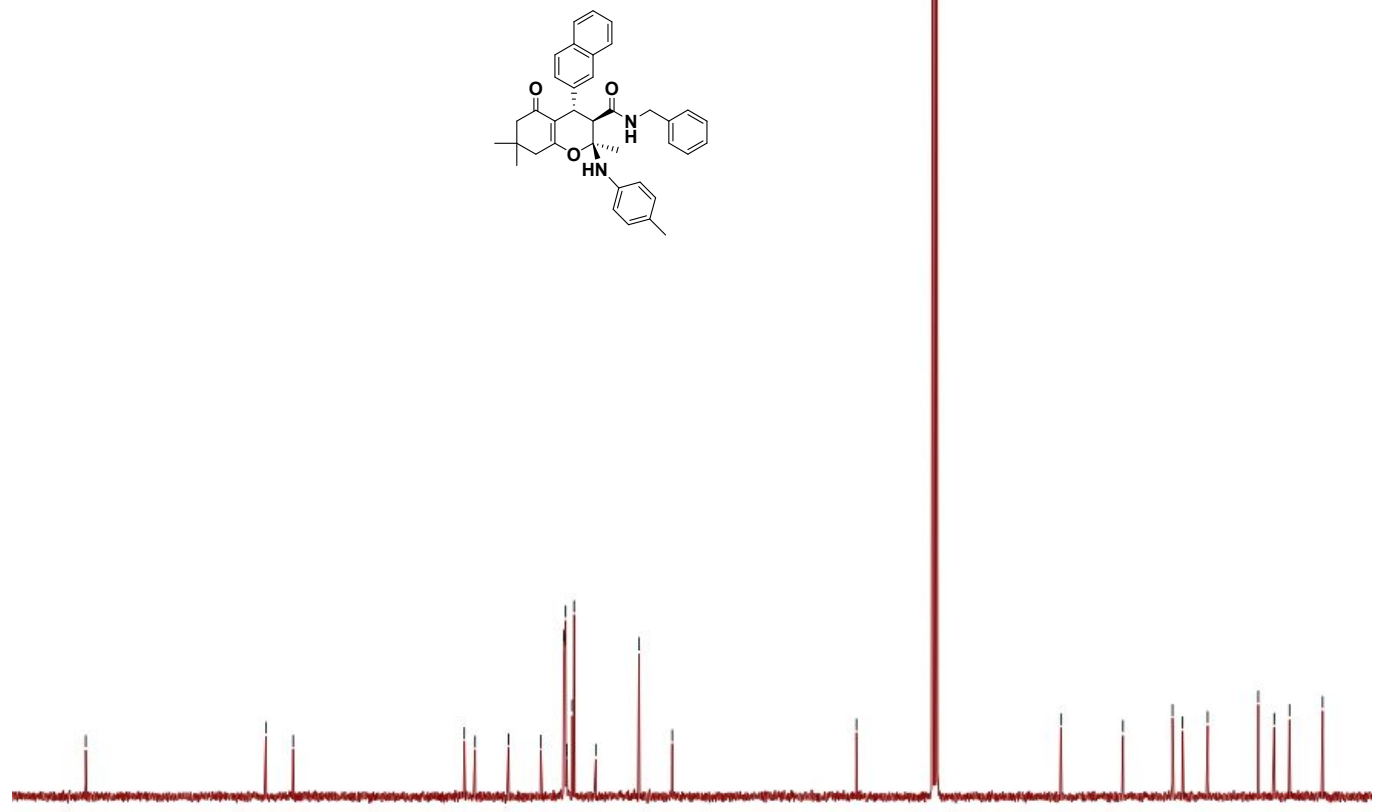

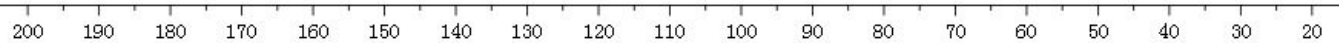



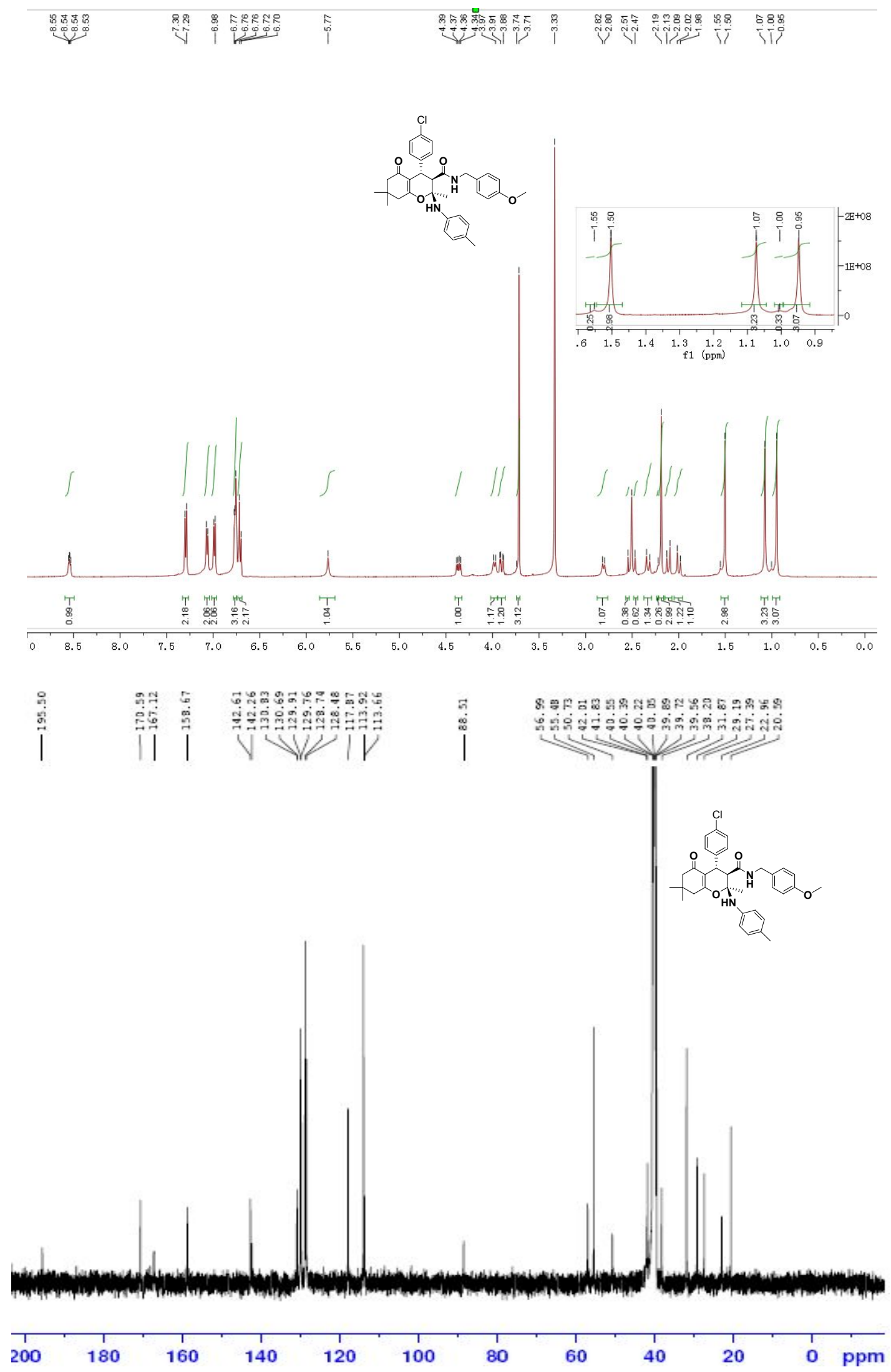


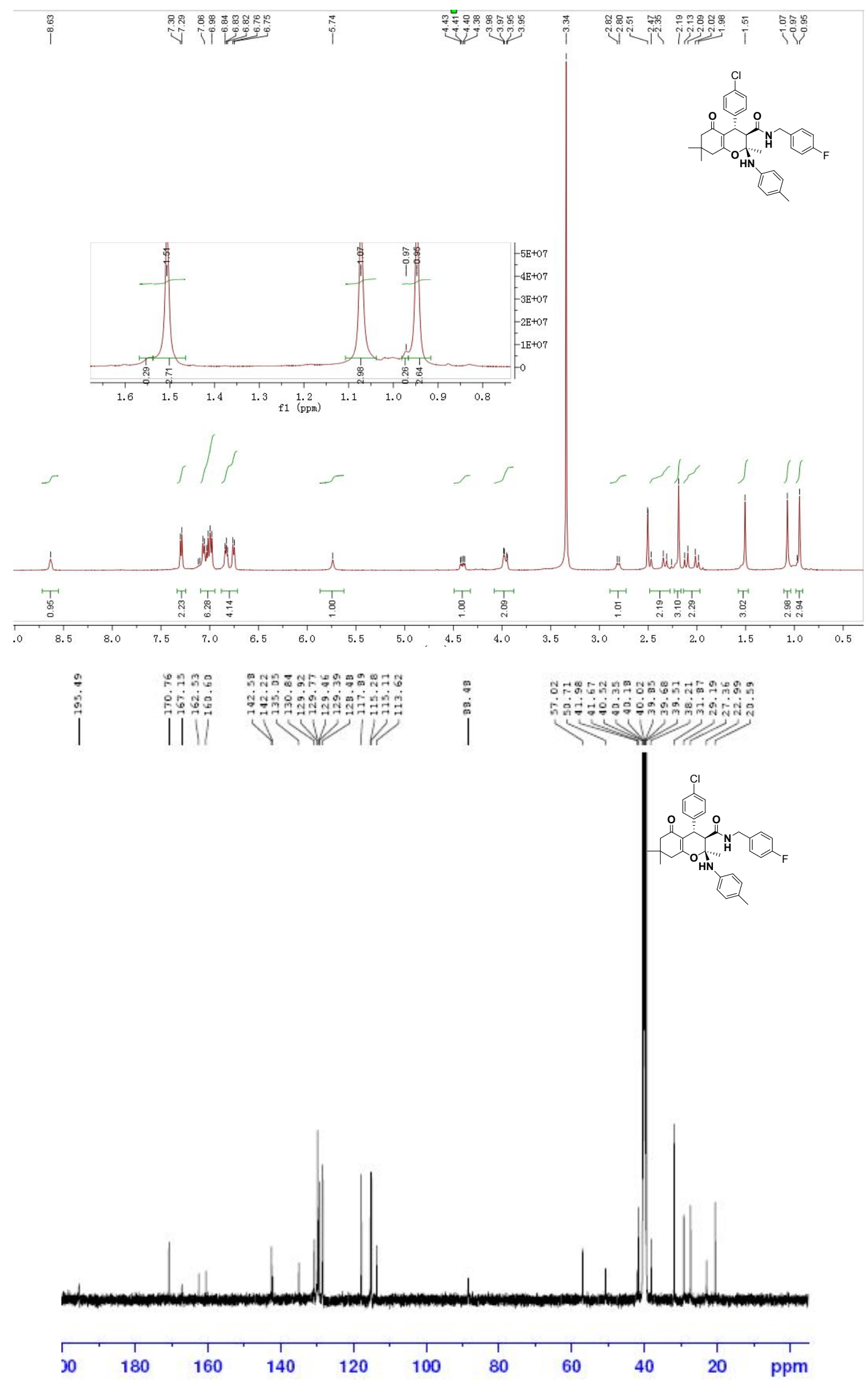




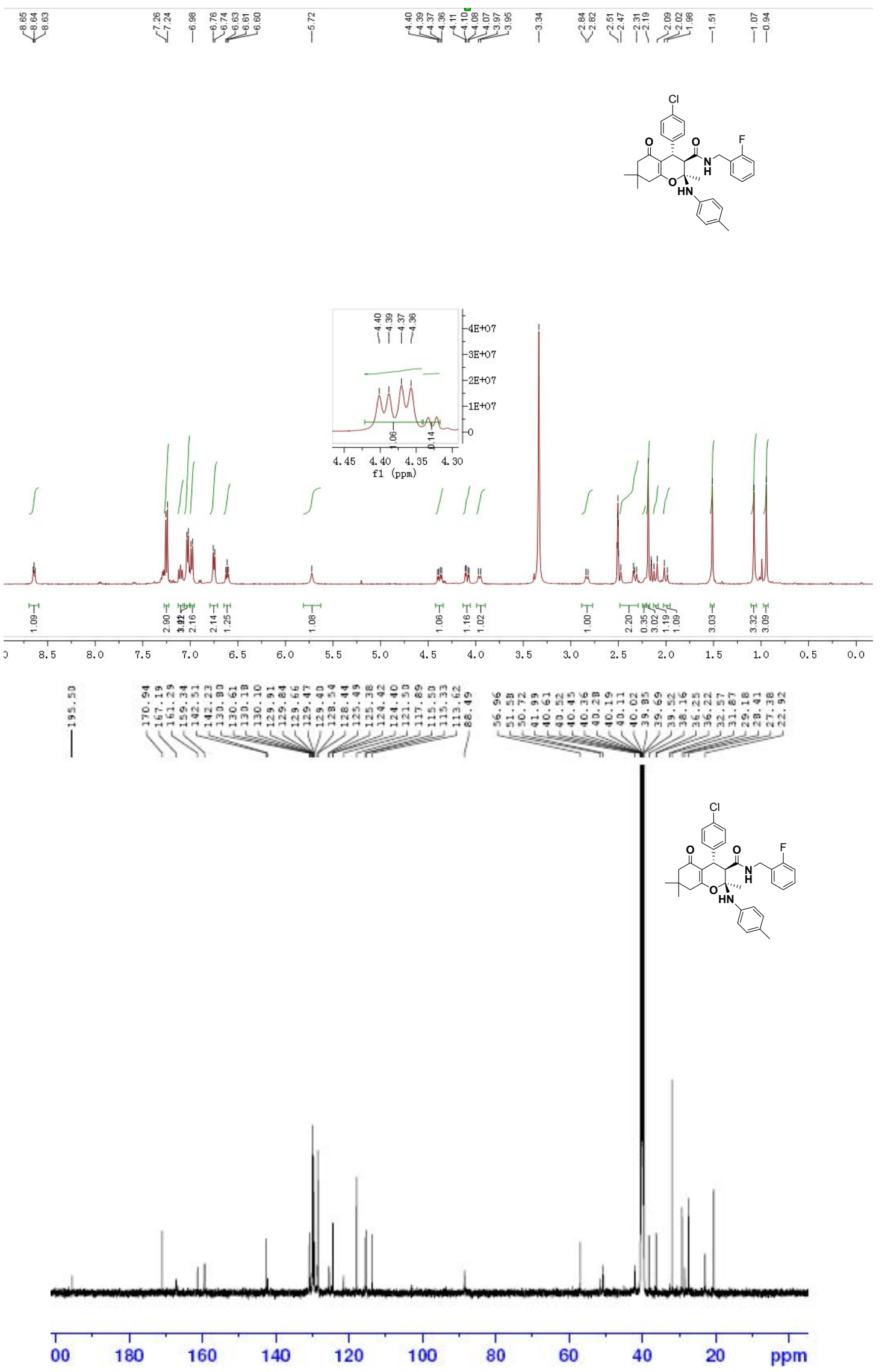




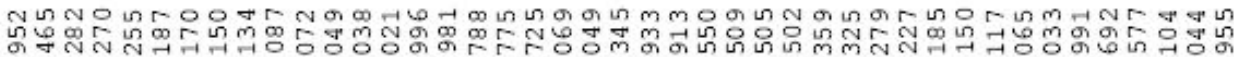

Grmprrming
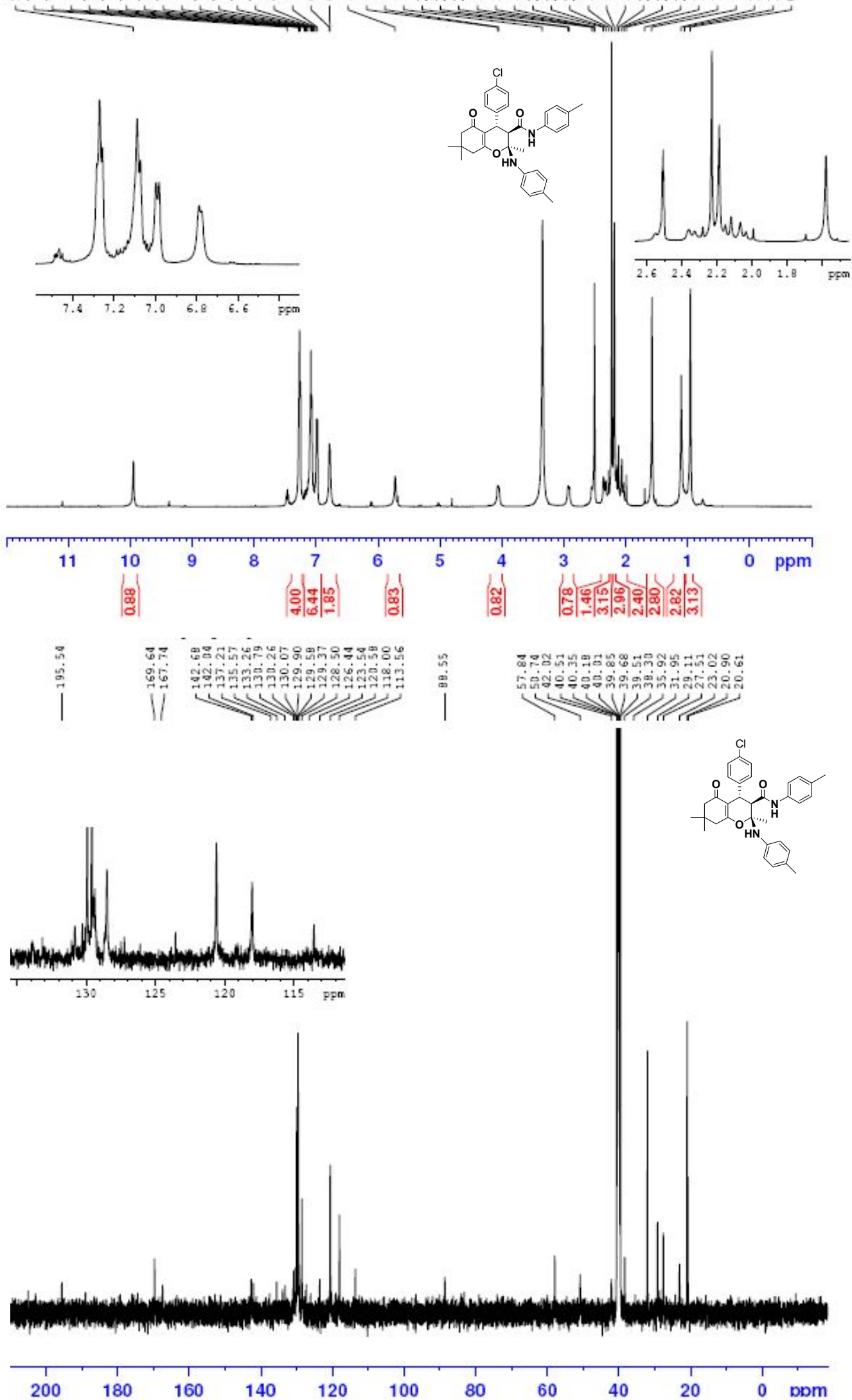

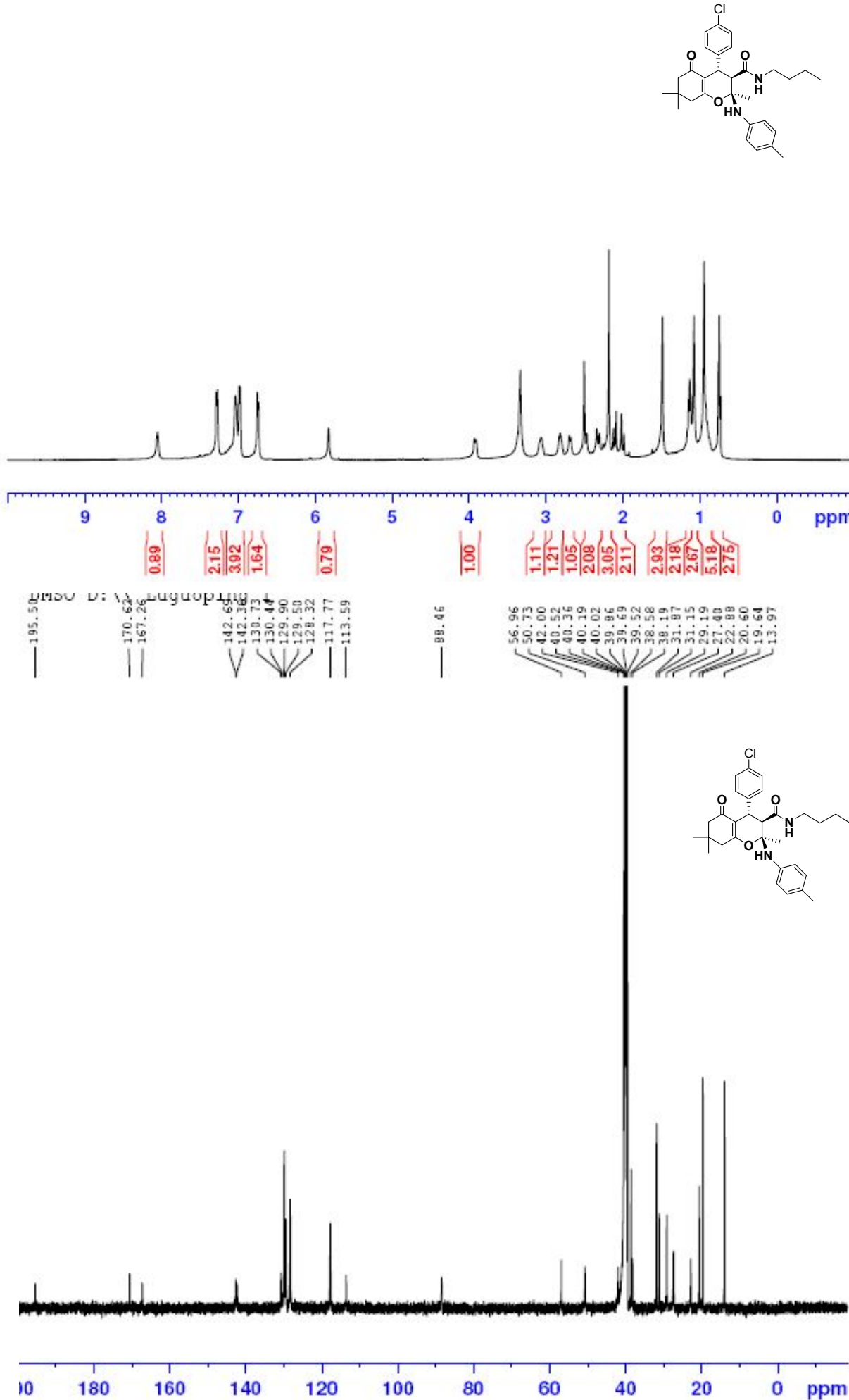

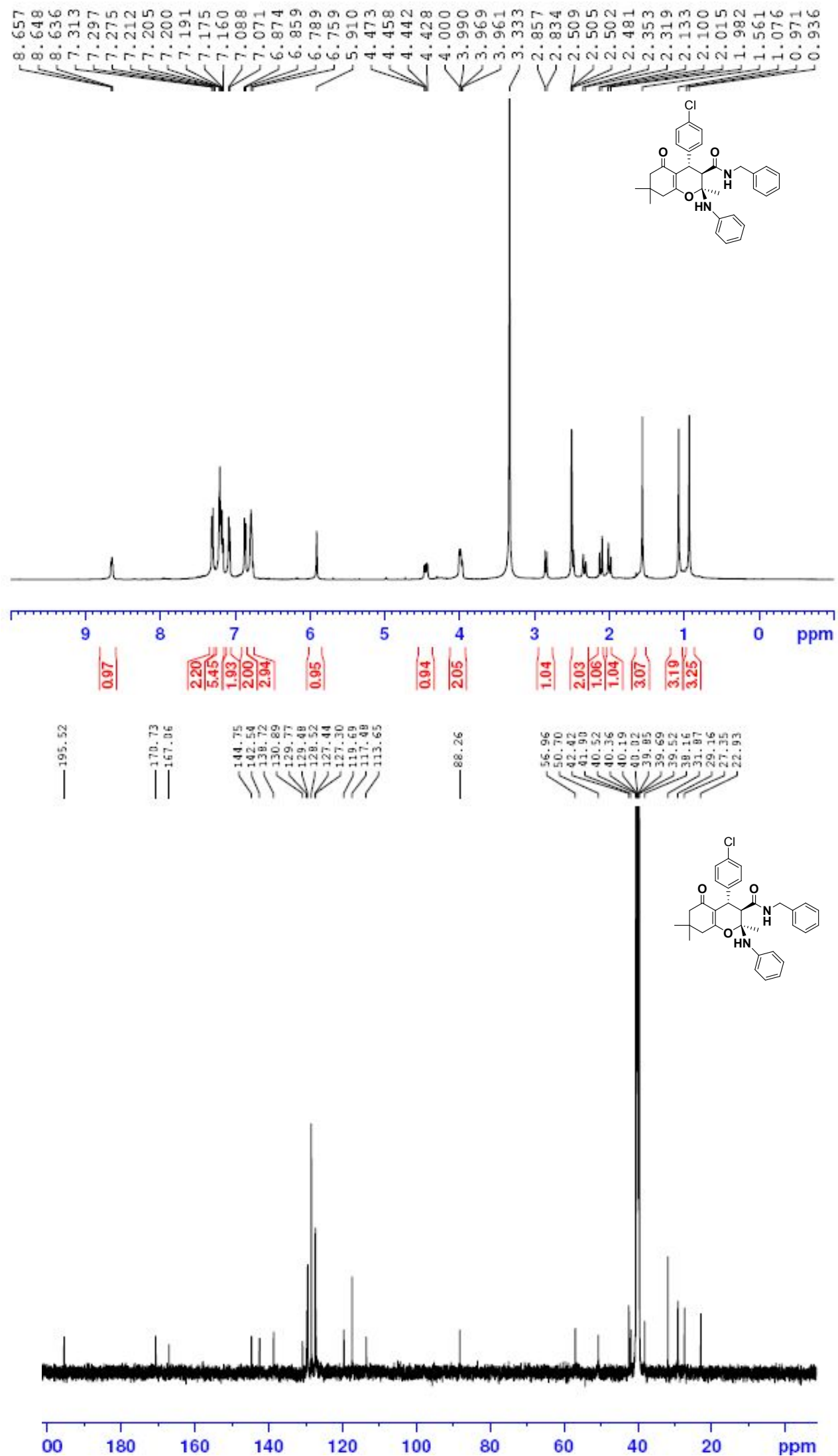


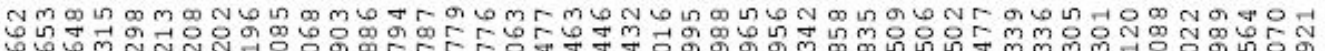

ம்

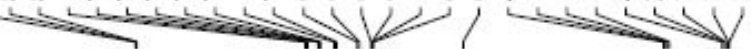
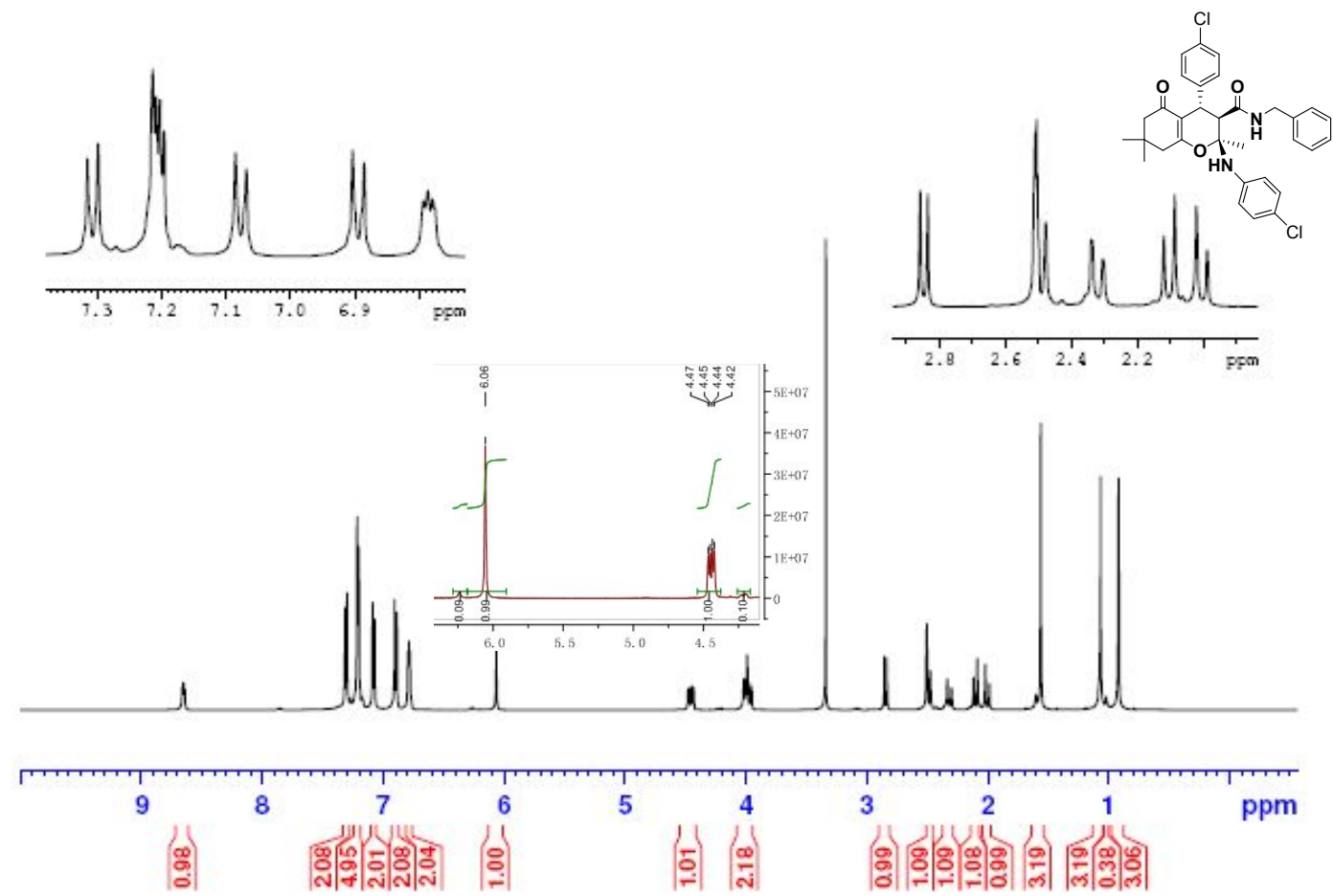

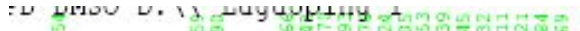
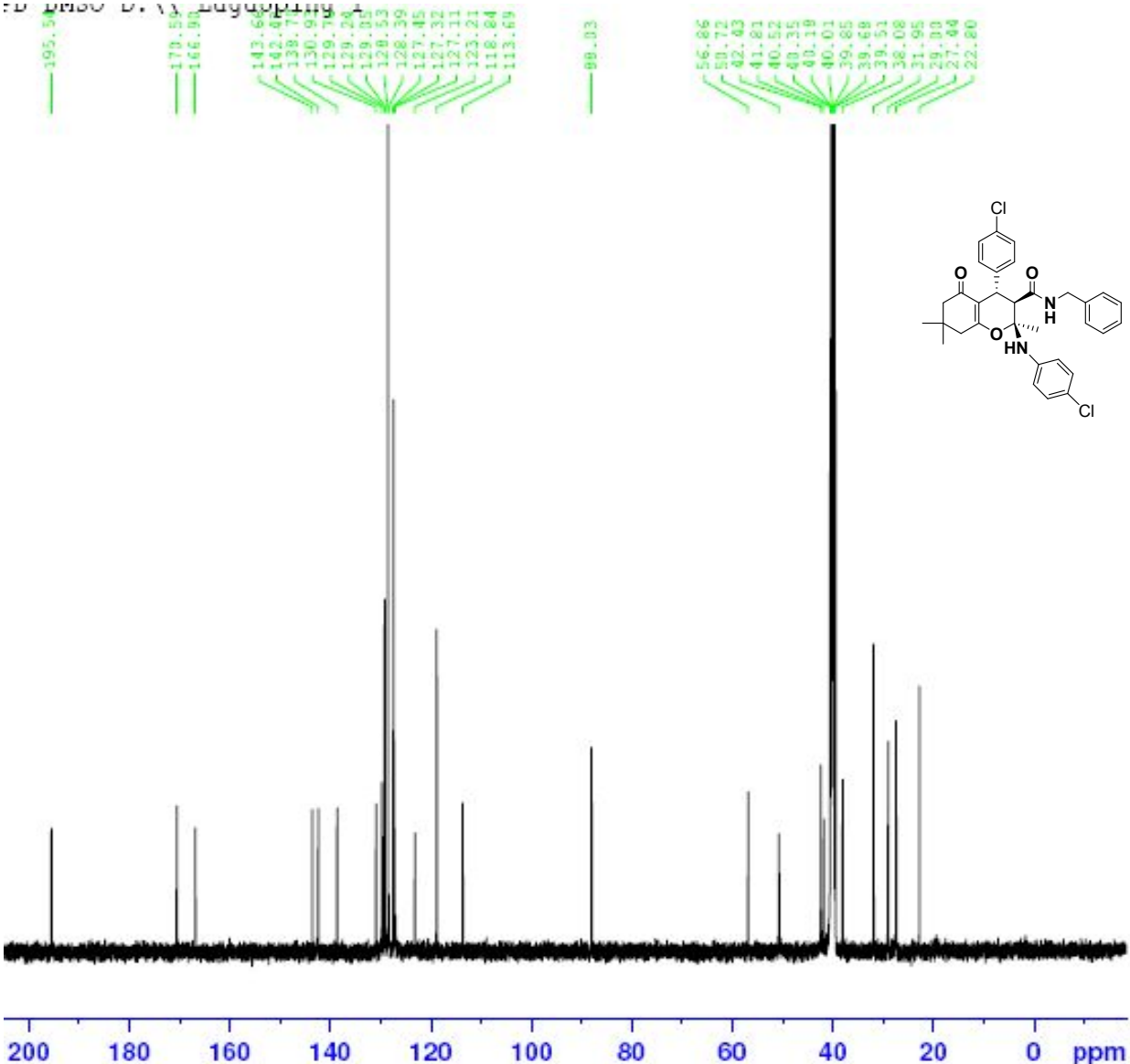


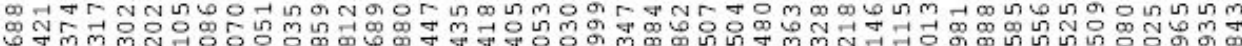

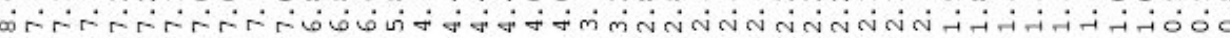

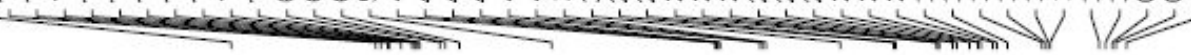

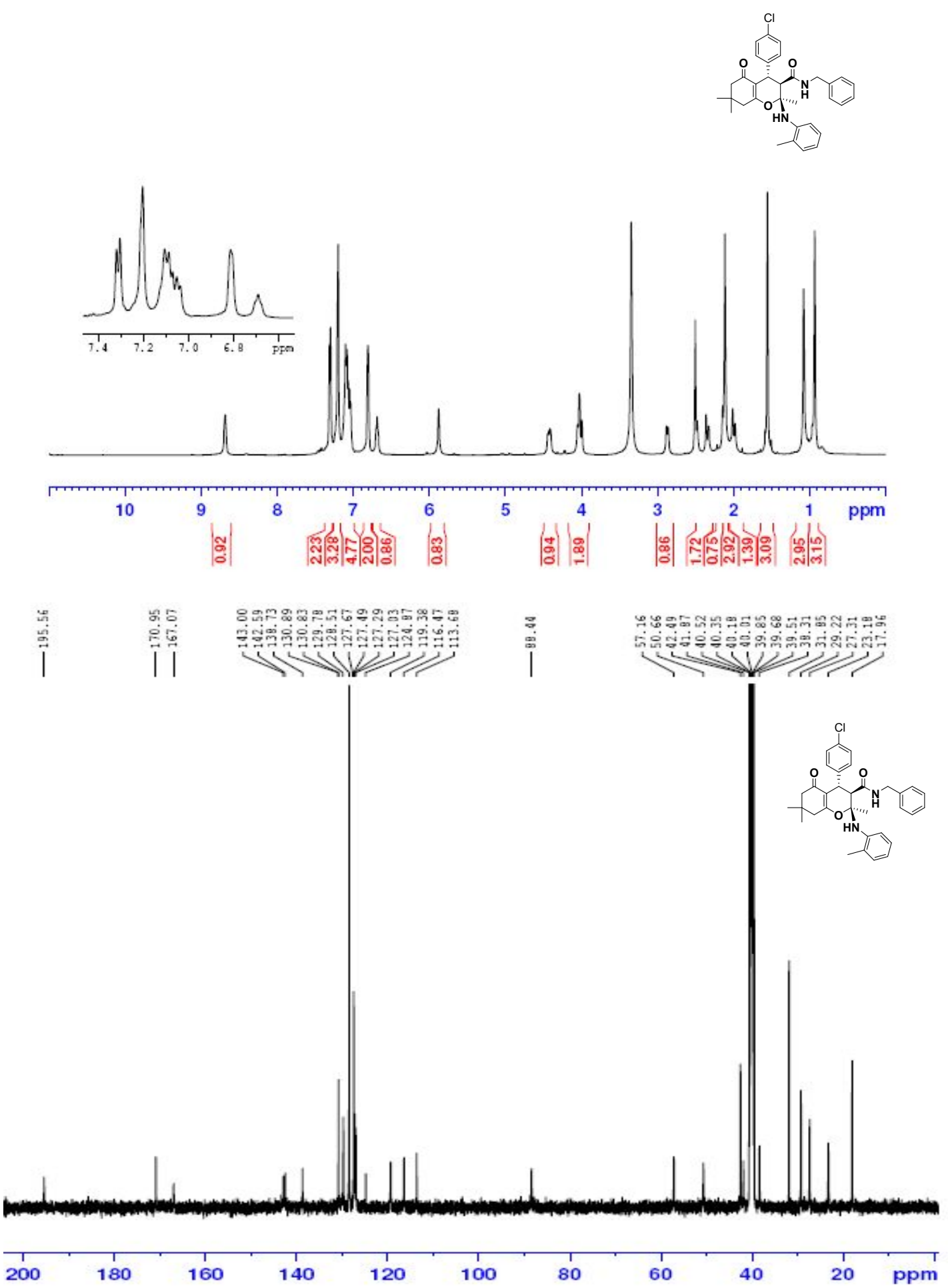



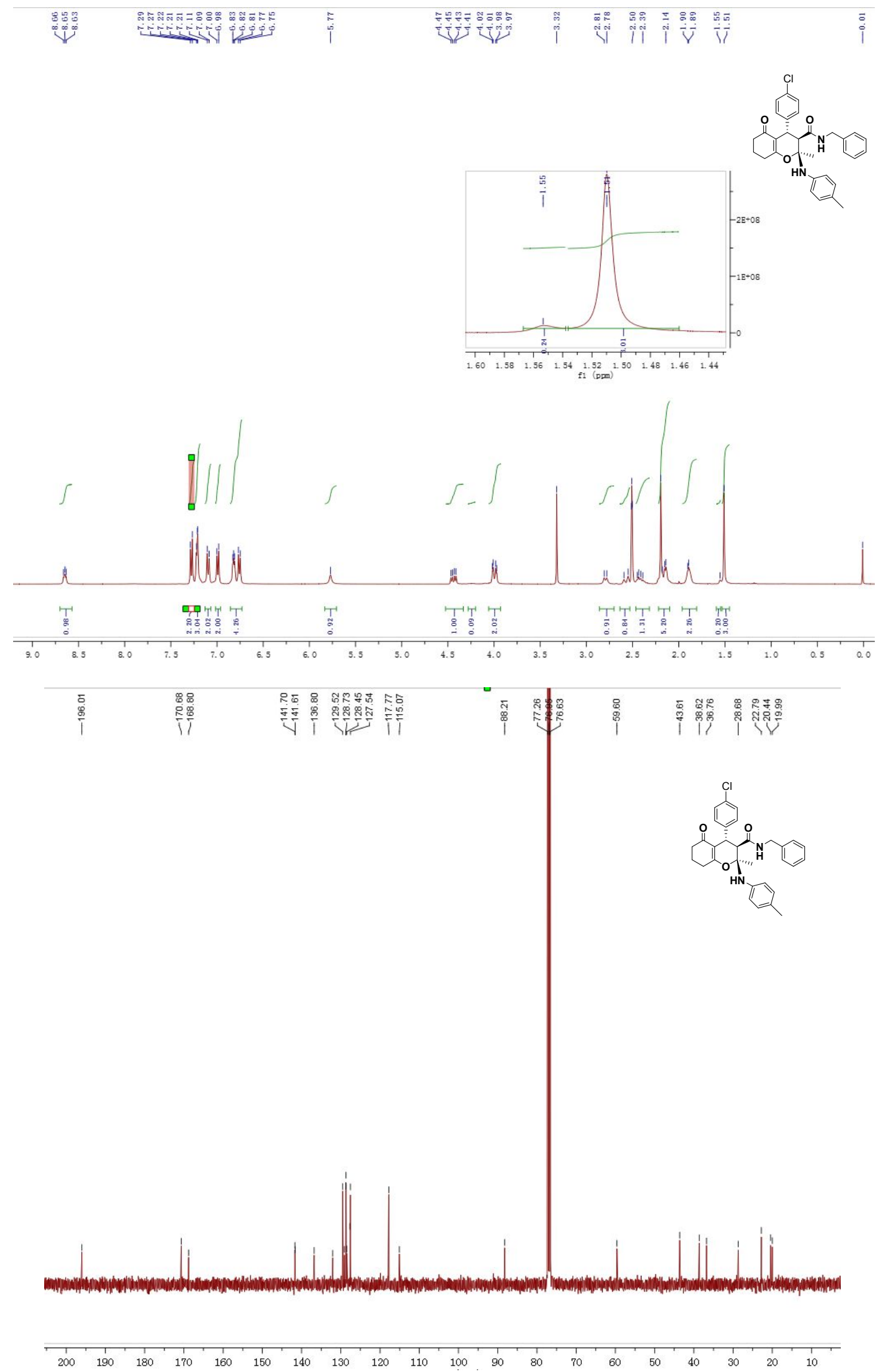


\begin{tabular}{|c|c|c|}
\hline 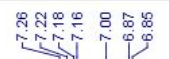 & $\begin{array}{l}\infty \\
0 \\
0\end{array}$ & 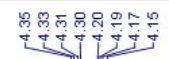 \\
\hline
\end{tabular}
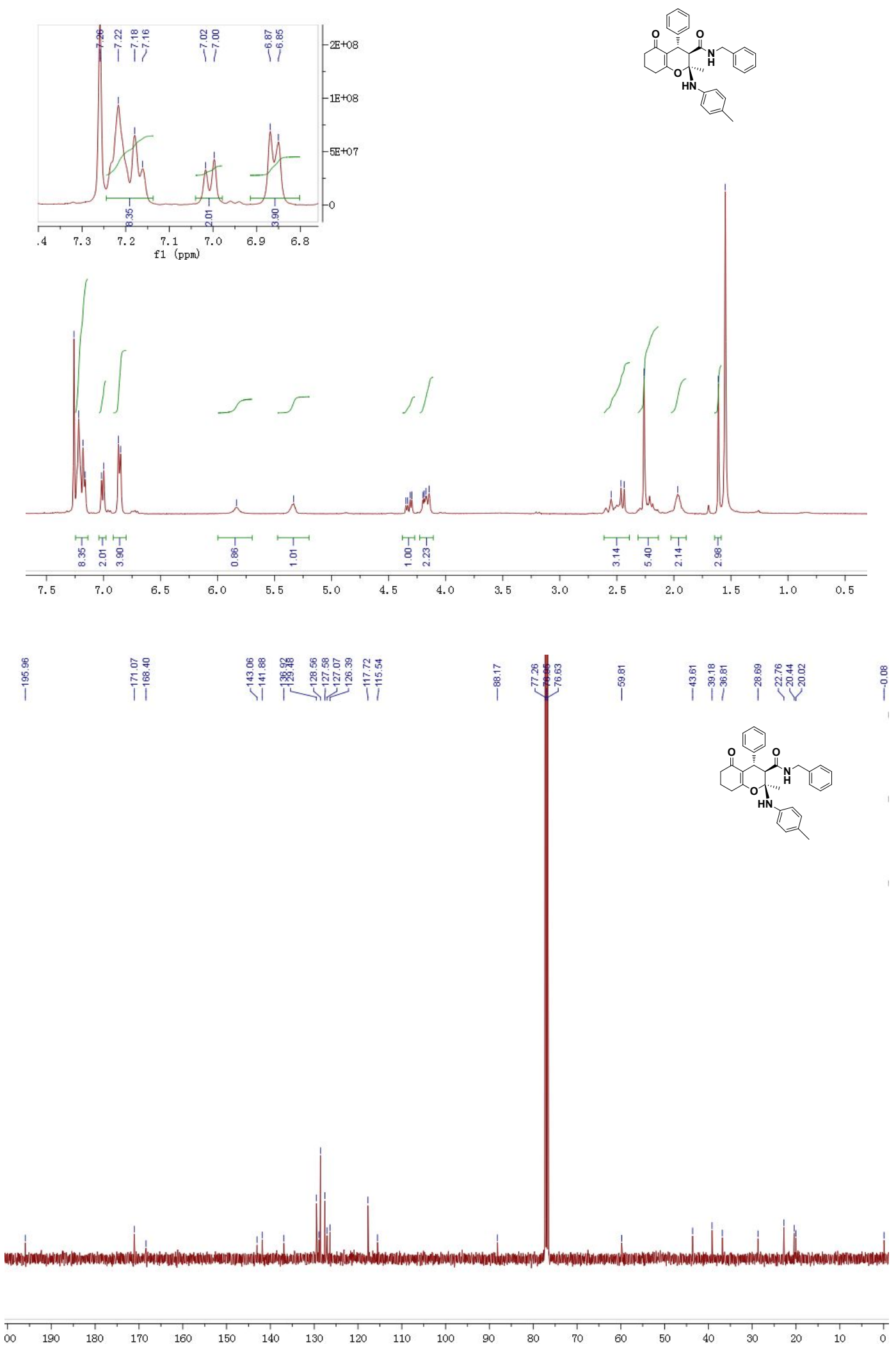

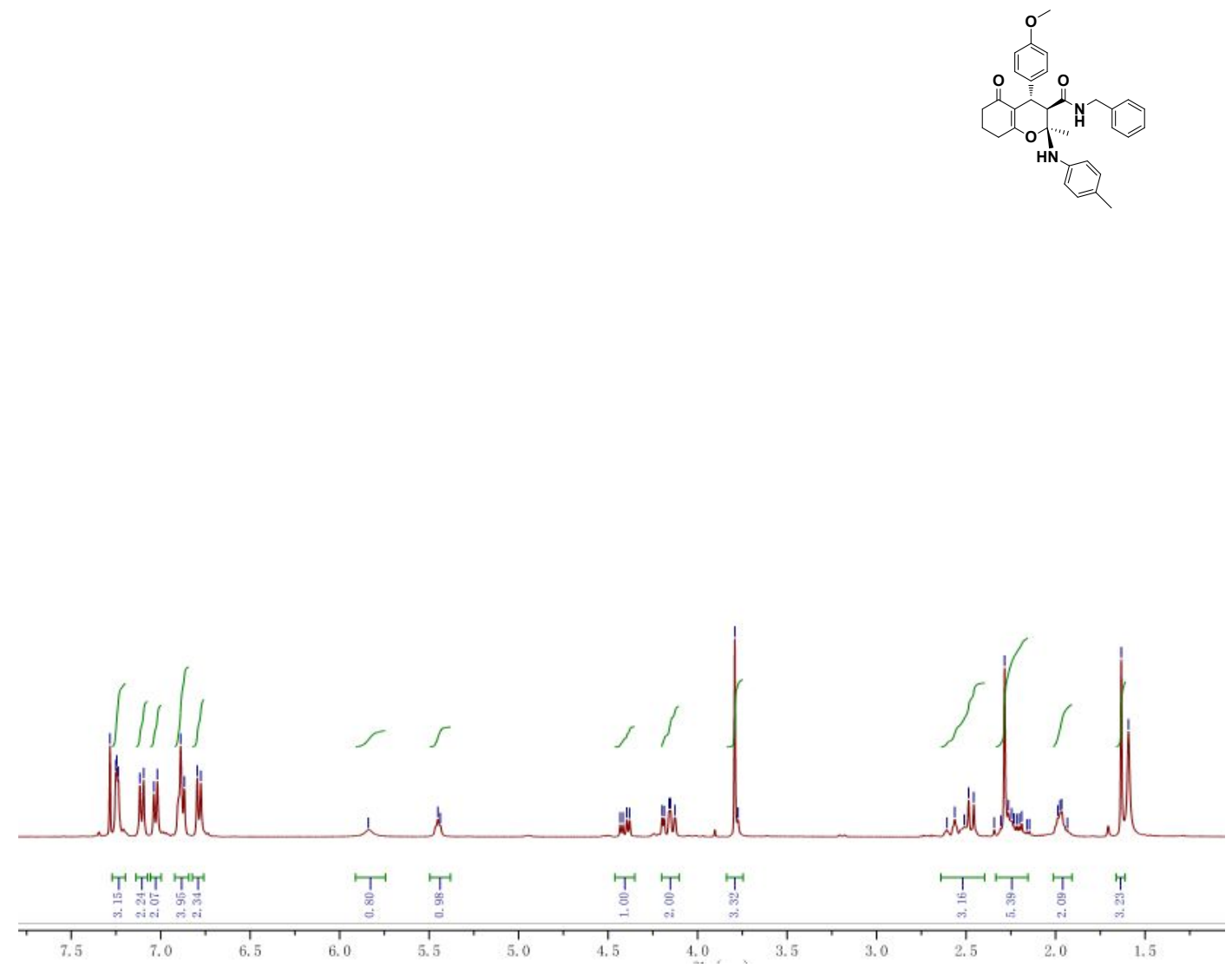

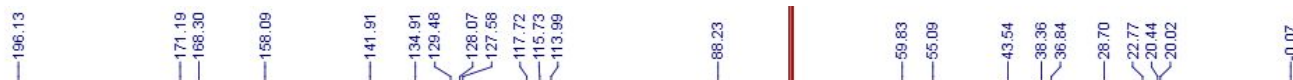
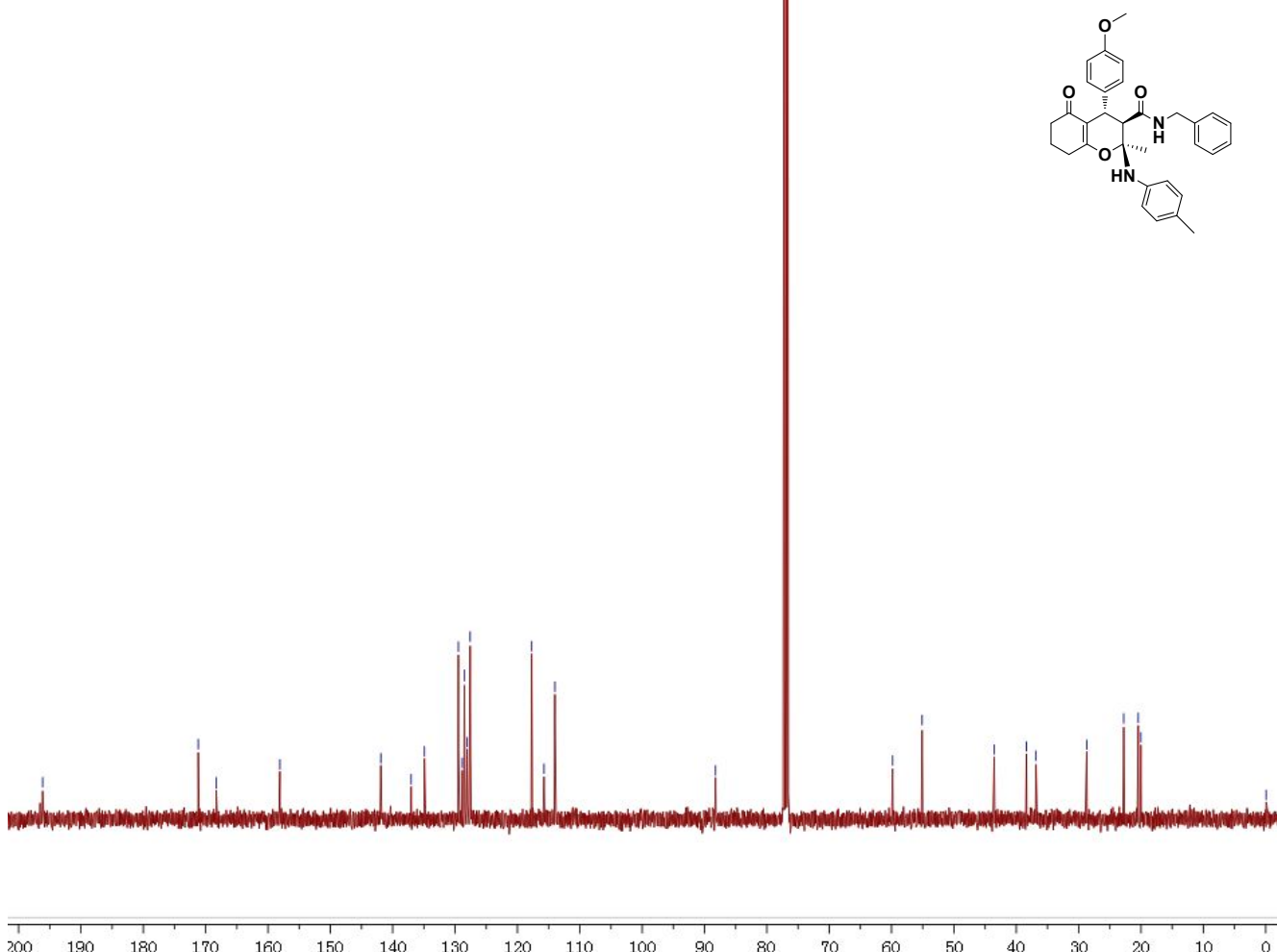
ע
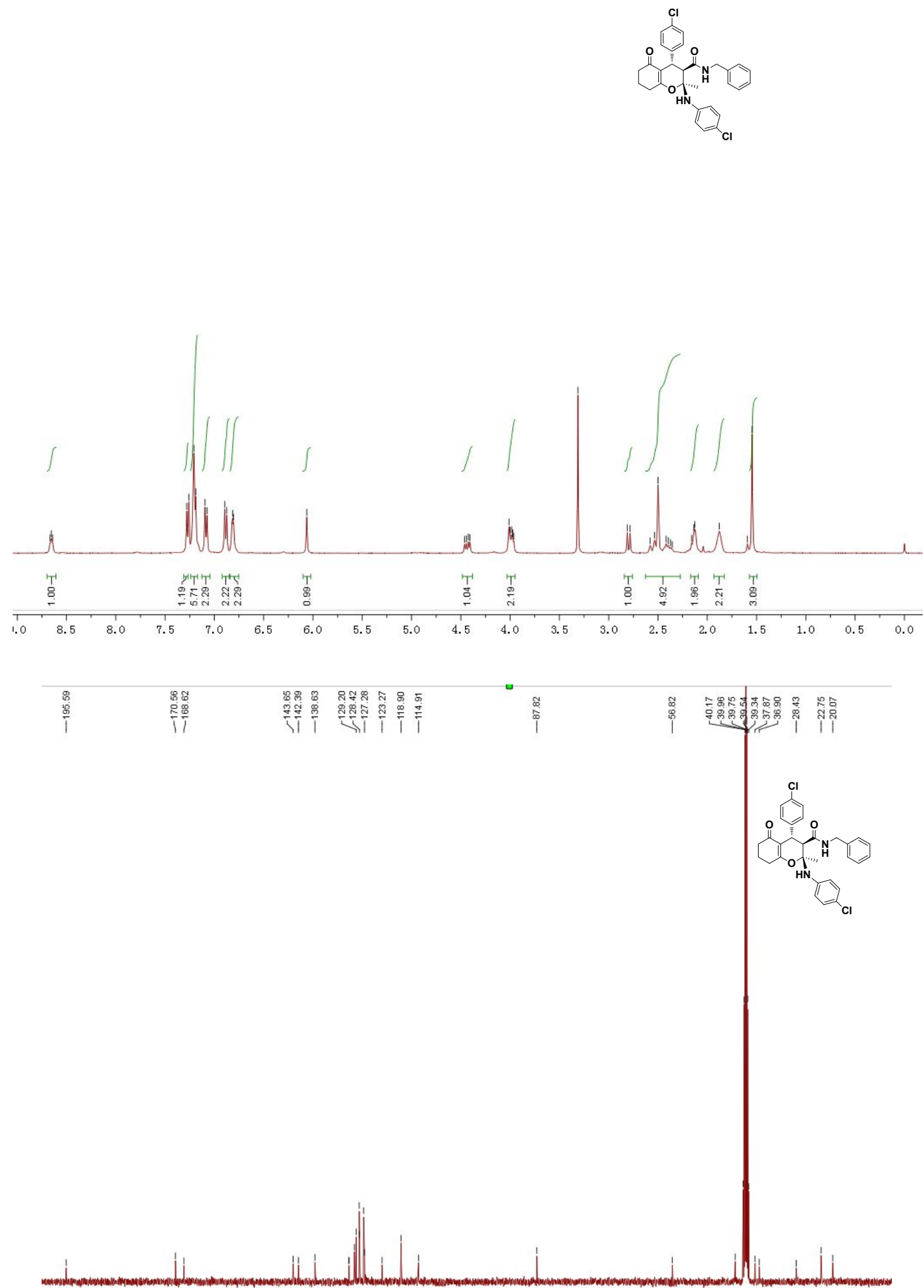

\begin{tabular}{llllllllllllllllllllllllllll}
\hline 00 & 190 & 180 & 170 & 160 & 150 & 140 & 130 & 120 & 110 & 100 & 90 & 80 & 70 & 60 & 50 & 40 & 10 & 1 & 1 \\
\hline
\end{tabular} 


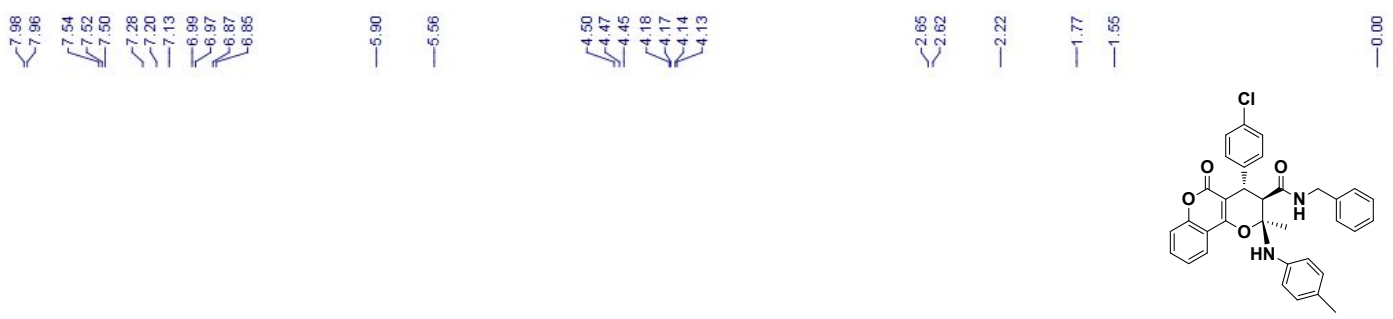

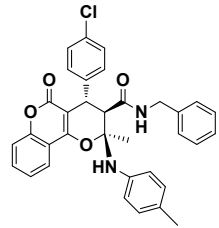

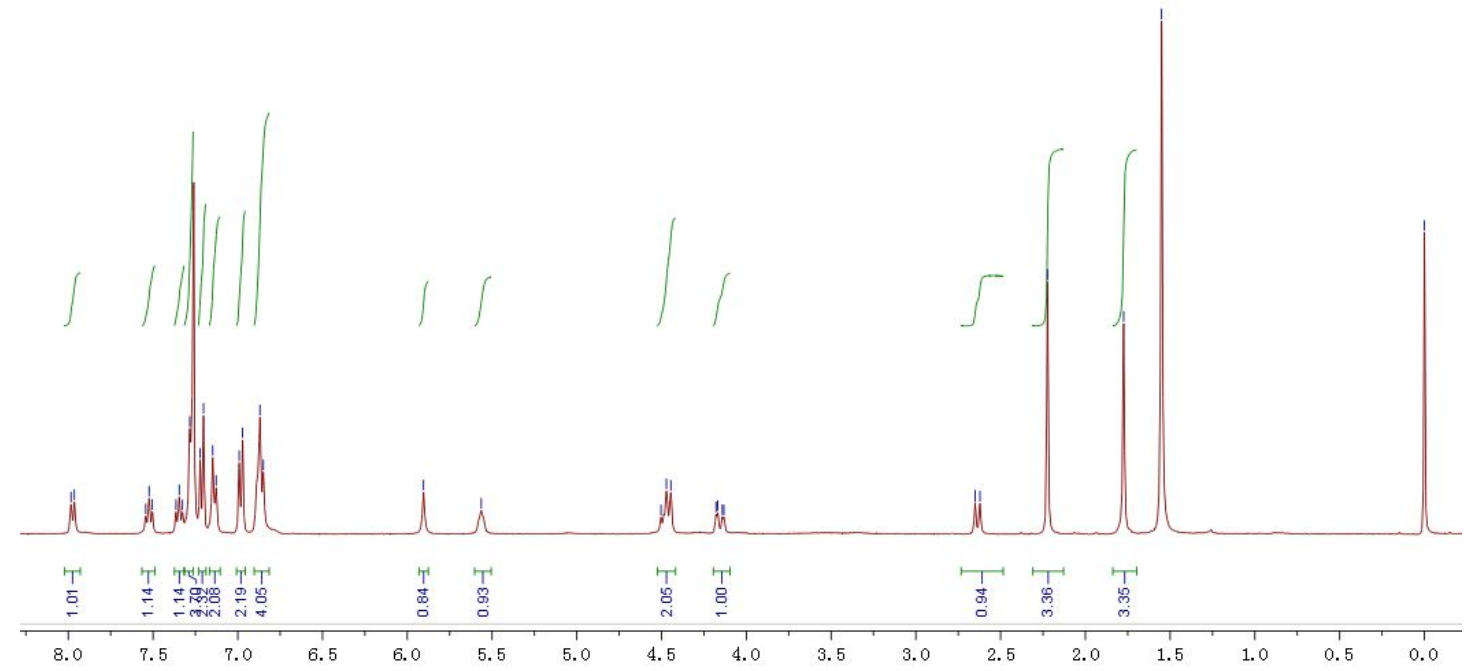

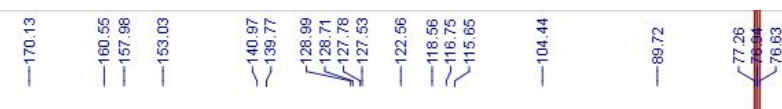

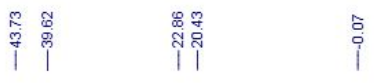

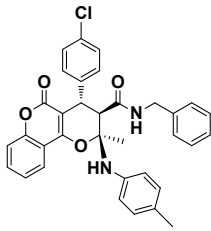

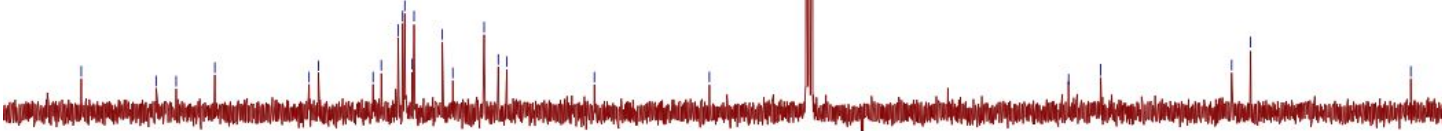

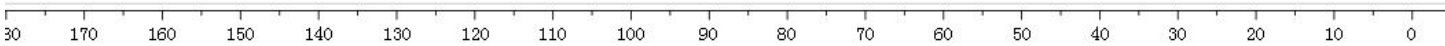




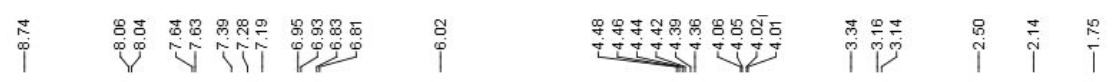
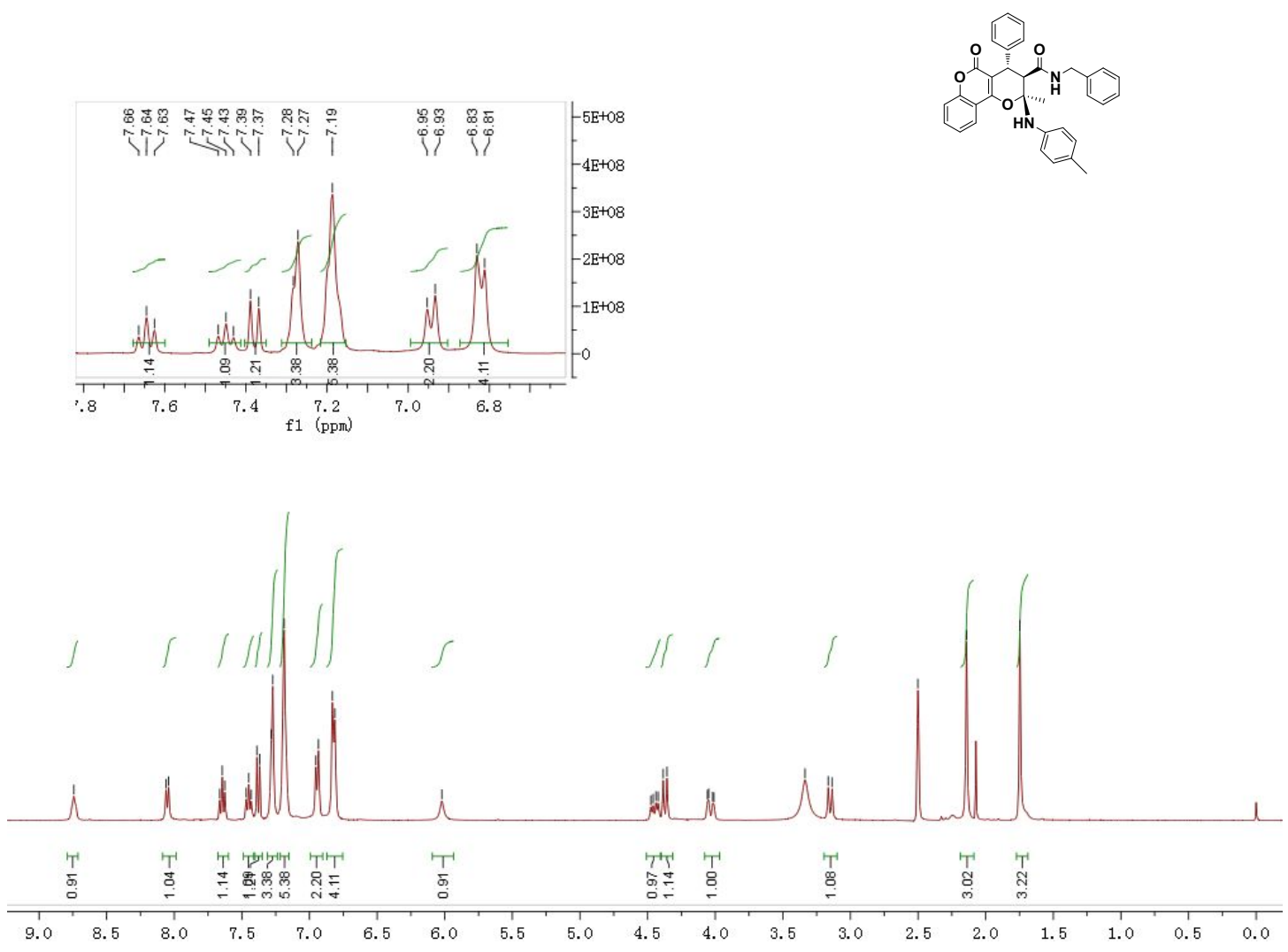

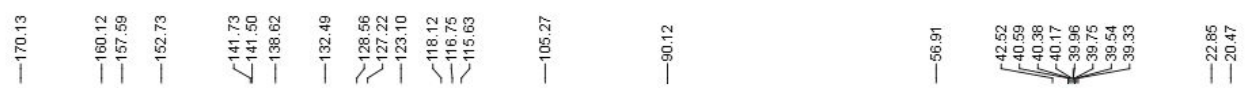
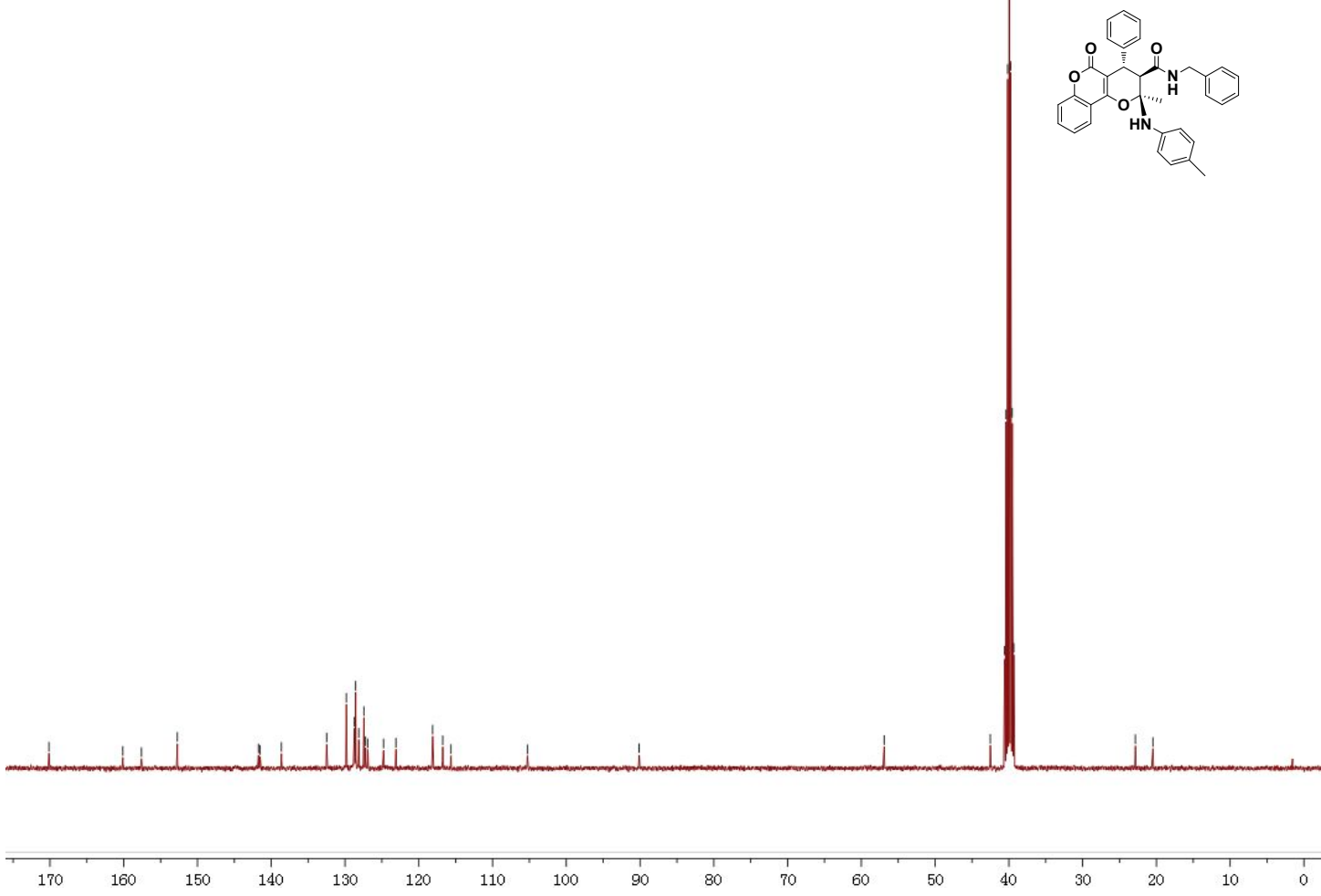


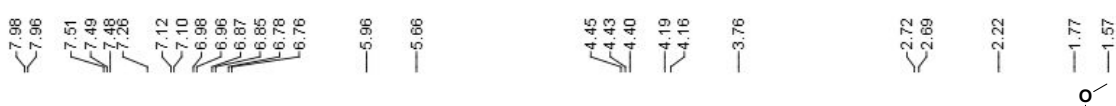
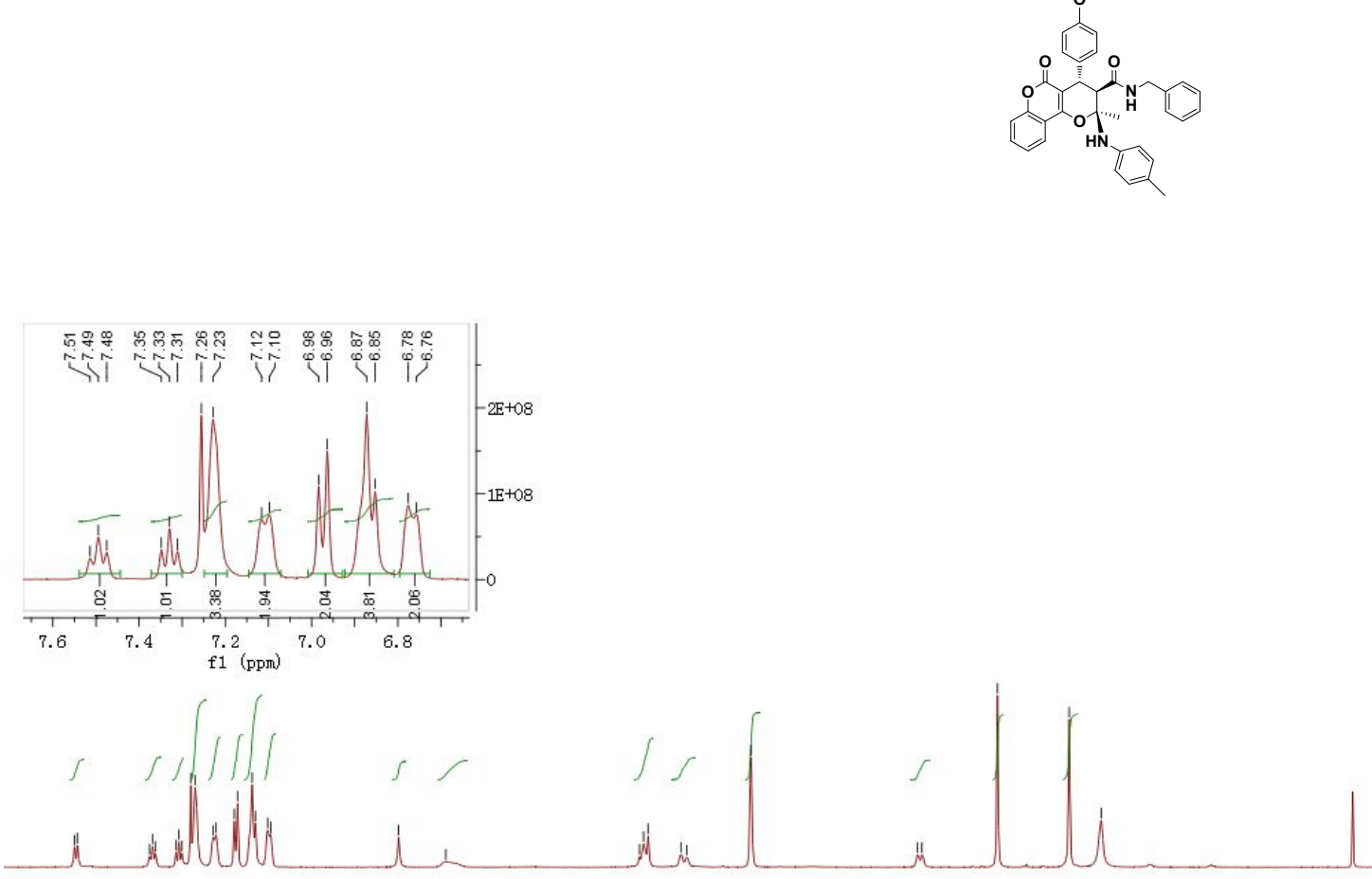

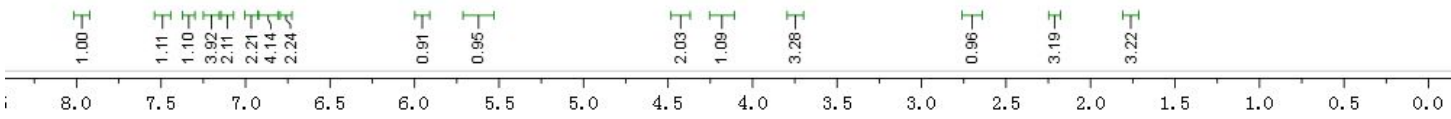

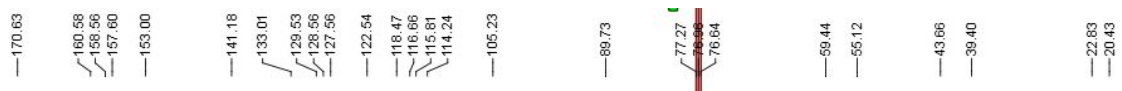
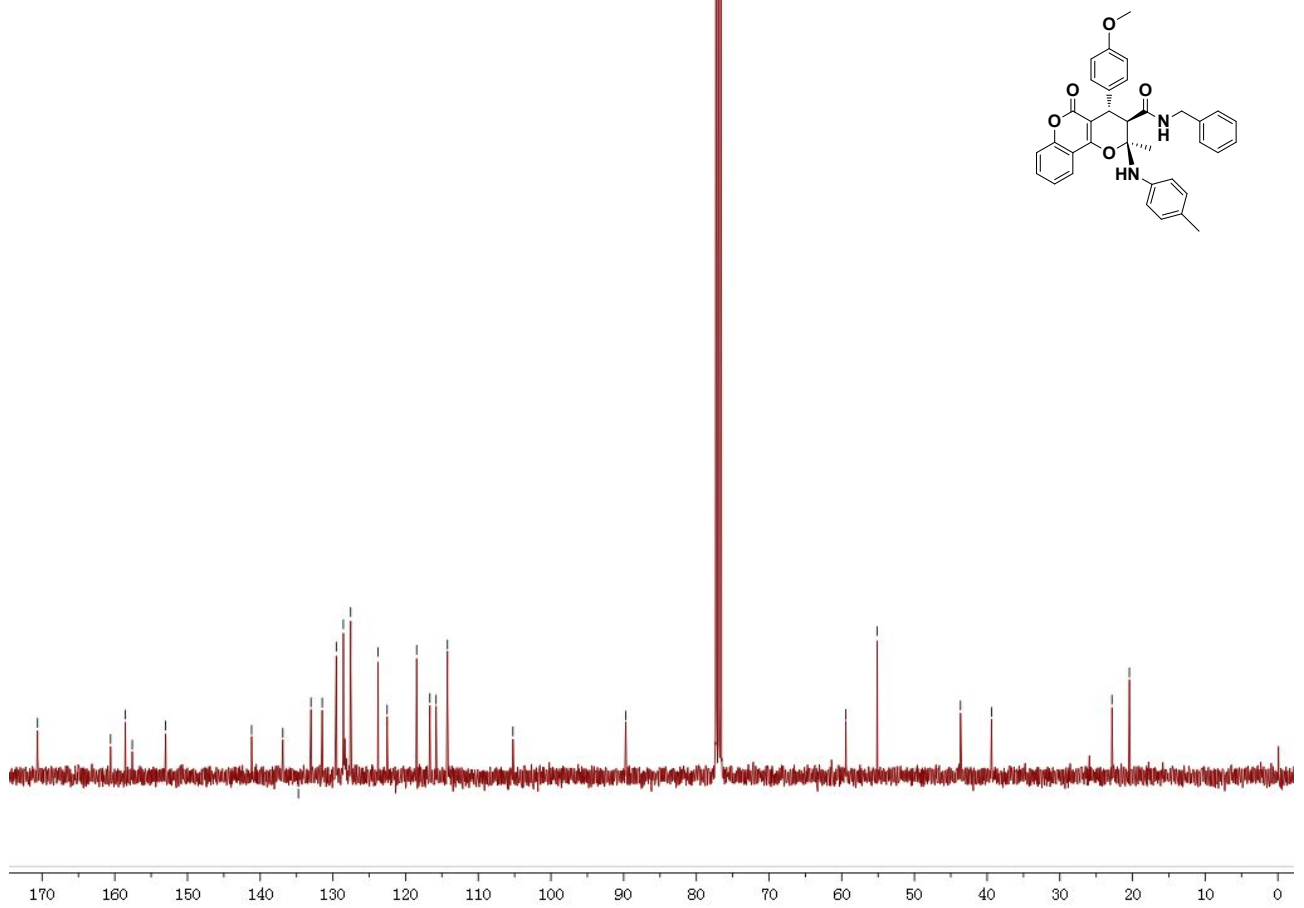


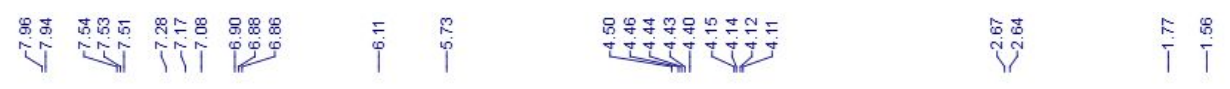
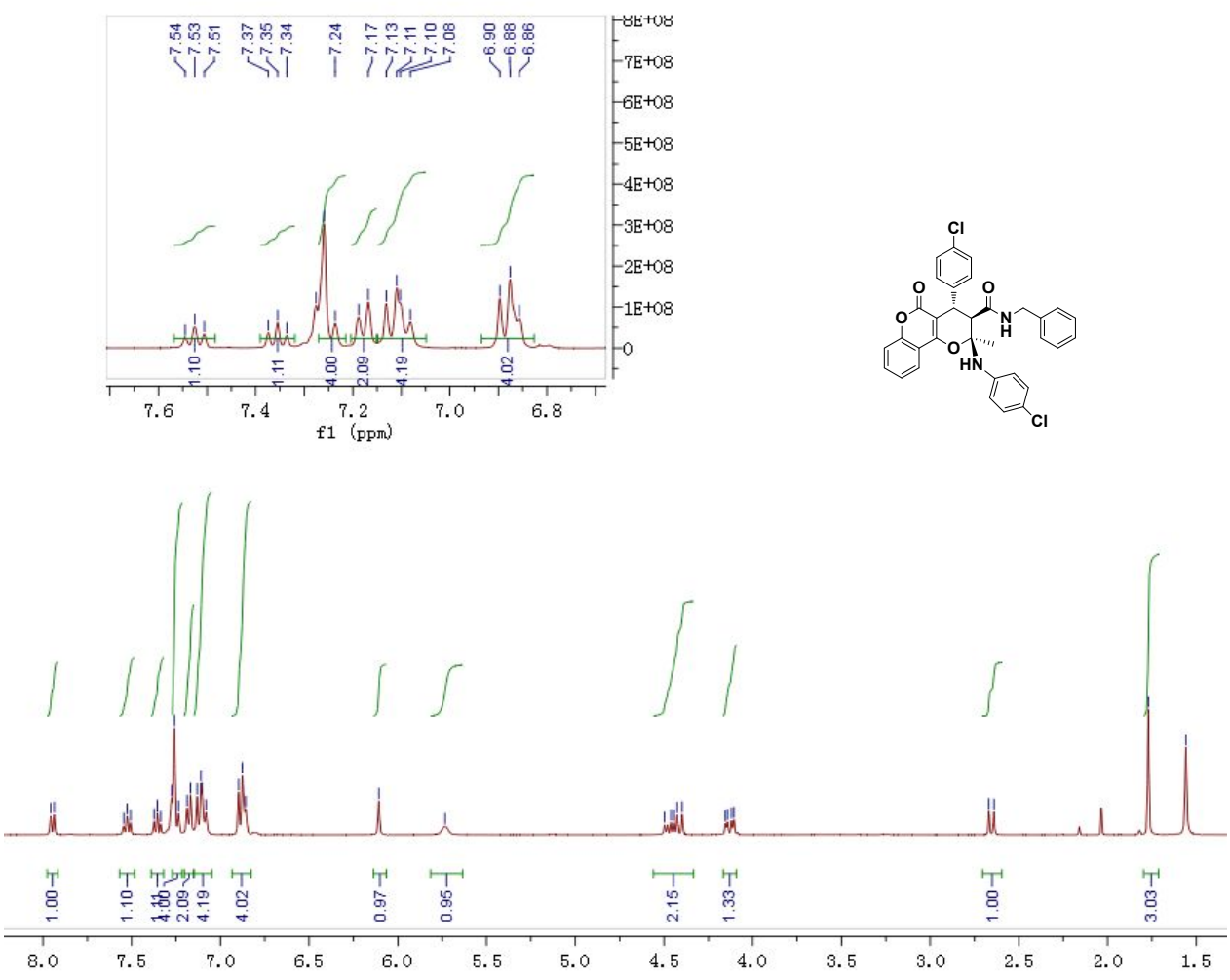

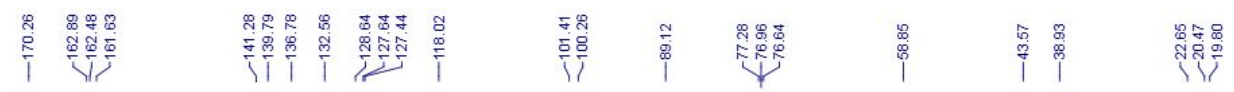
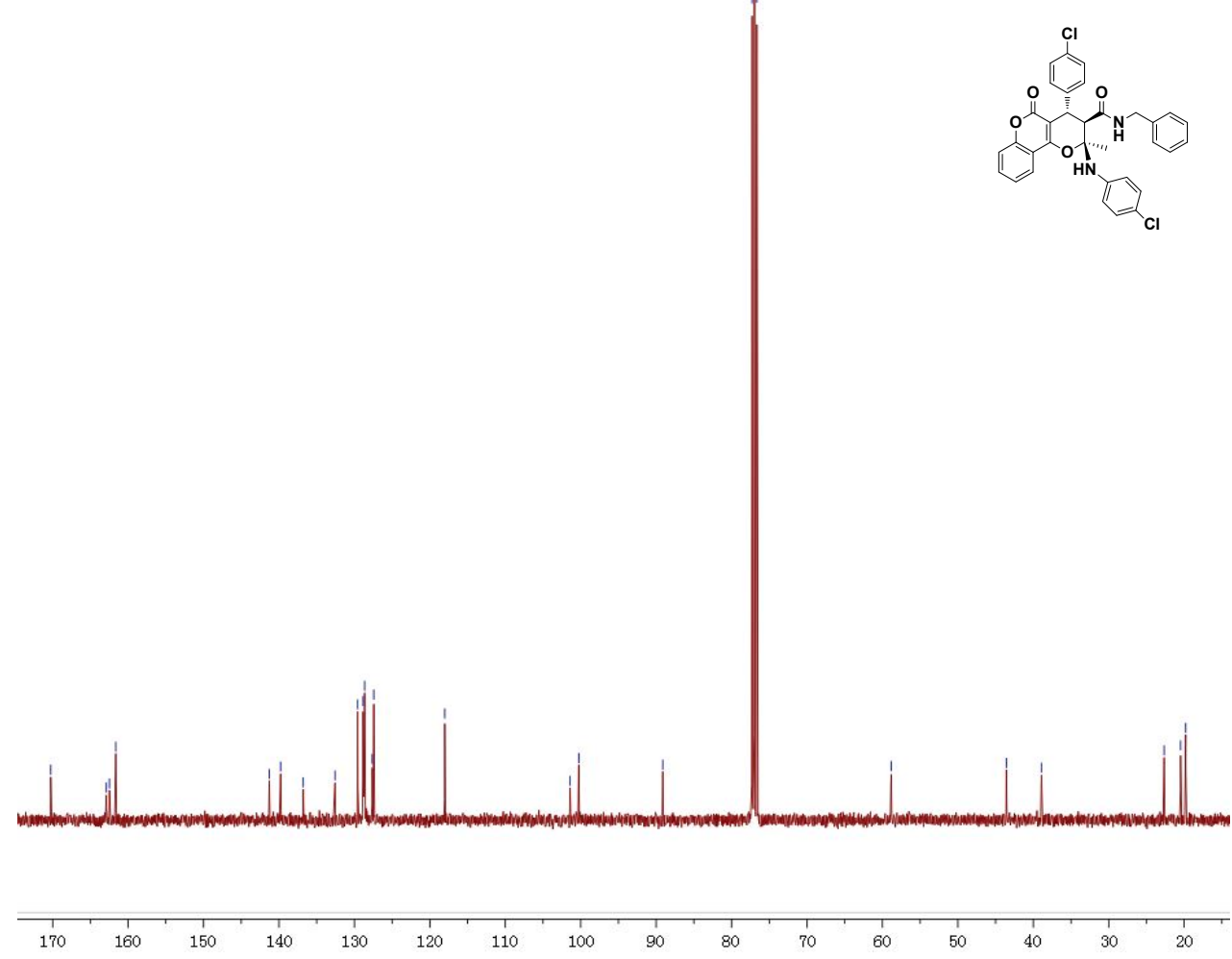

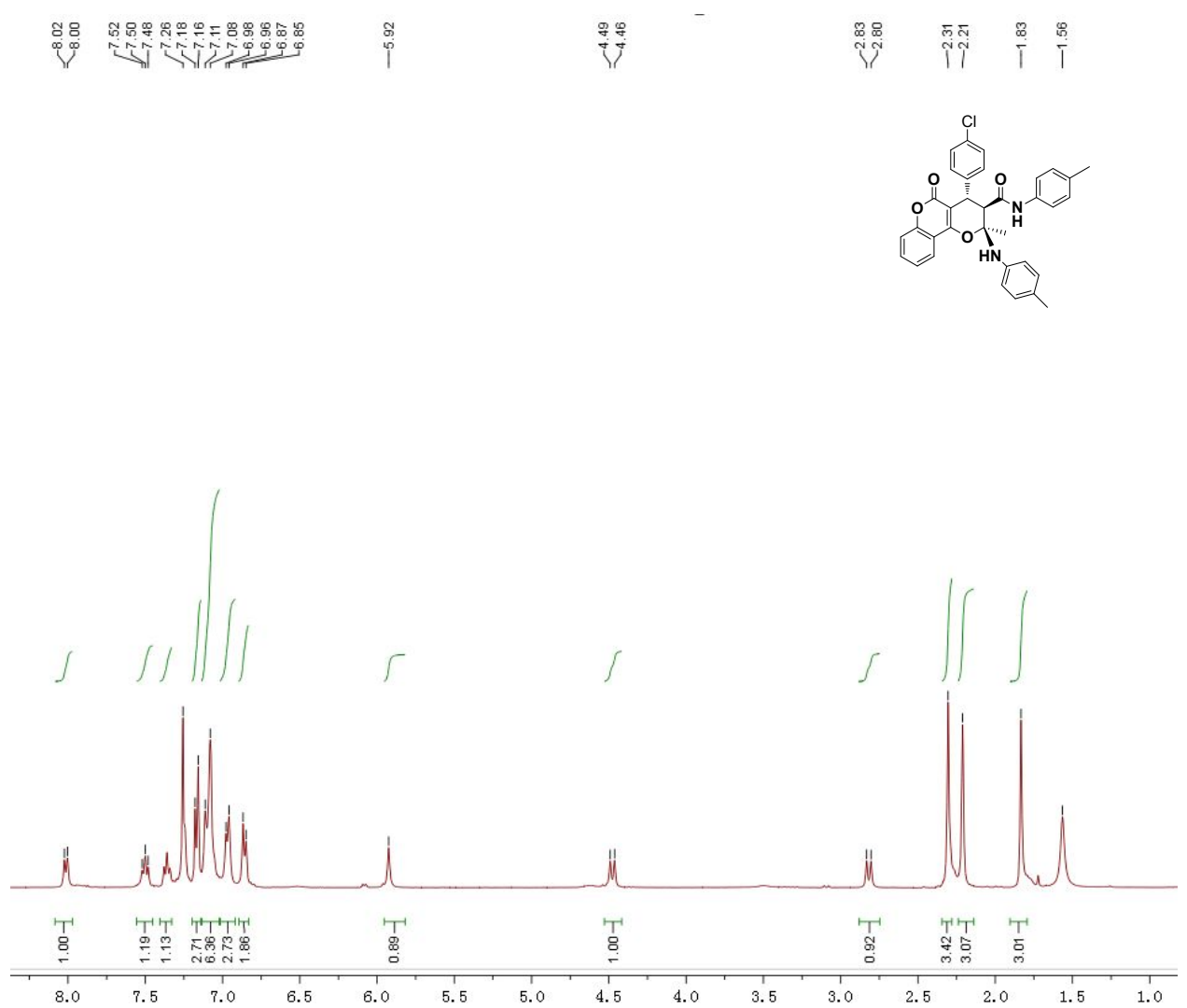

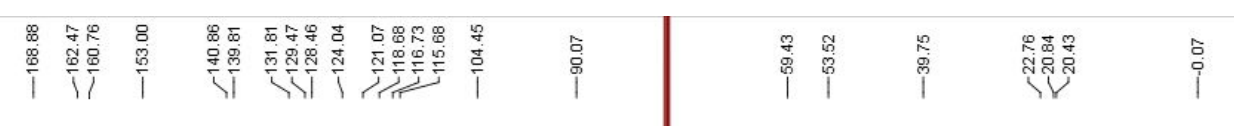
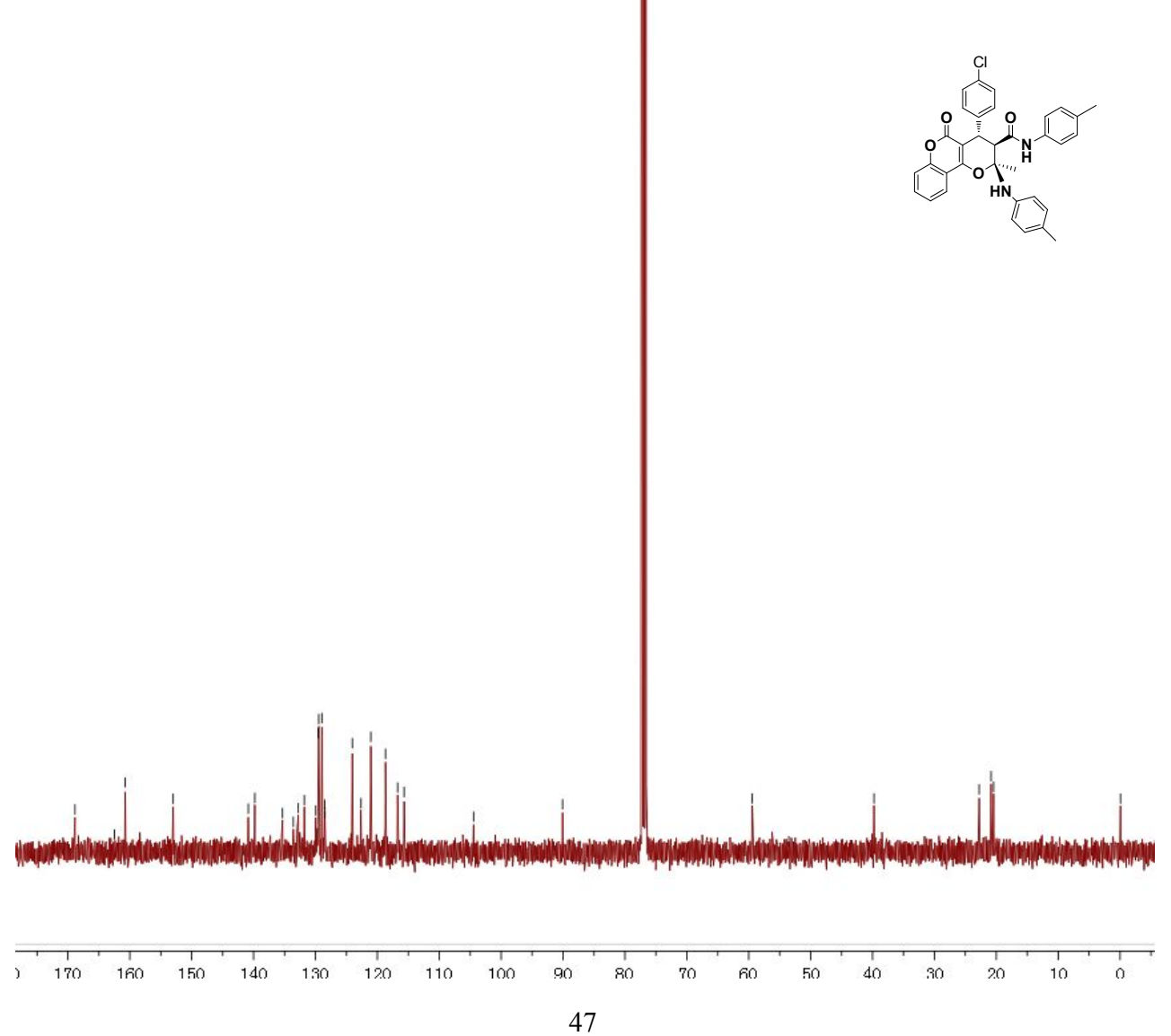

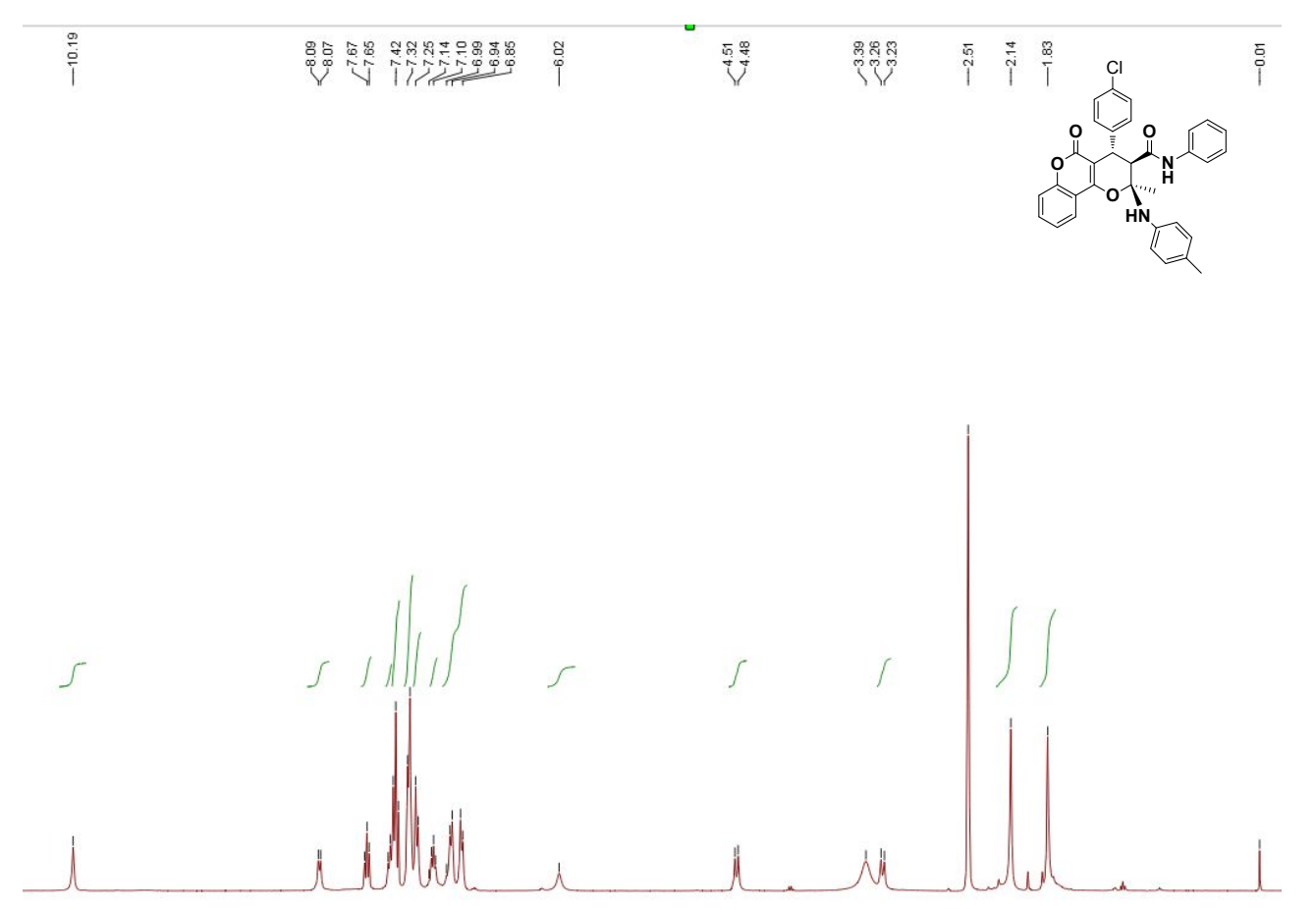

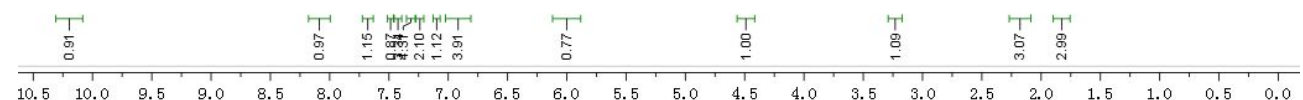

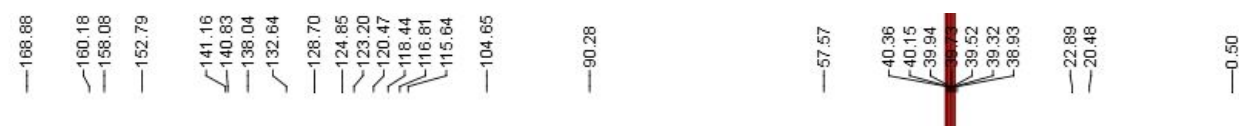
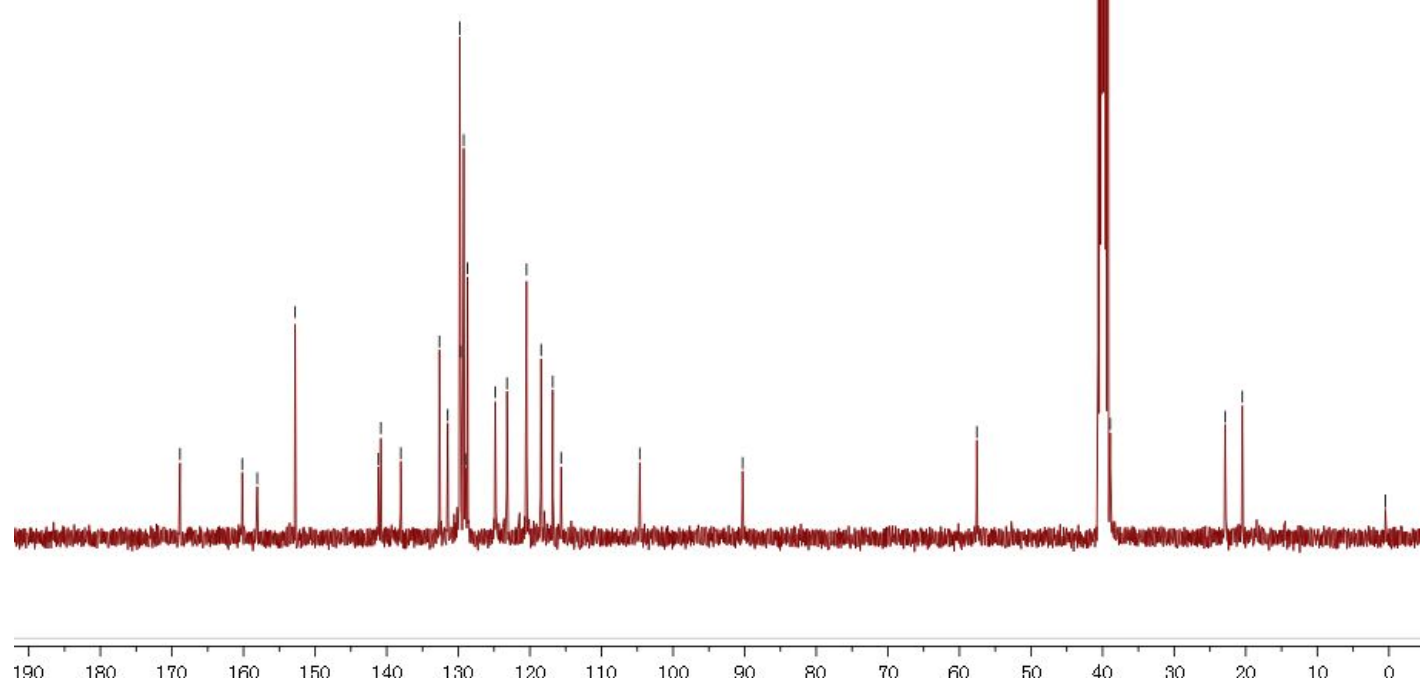

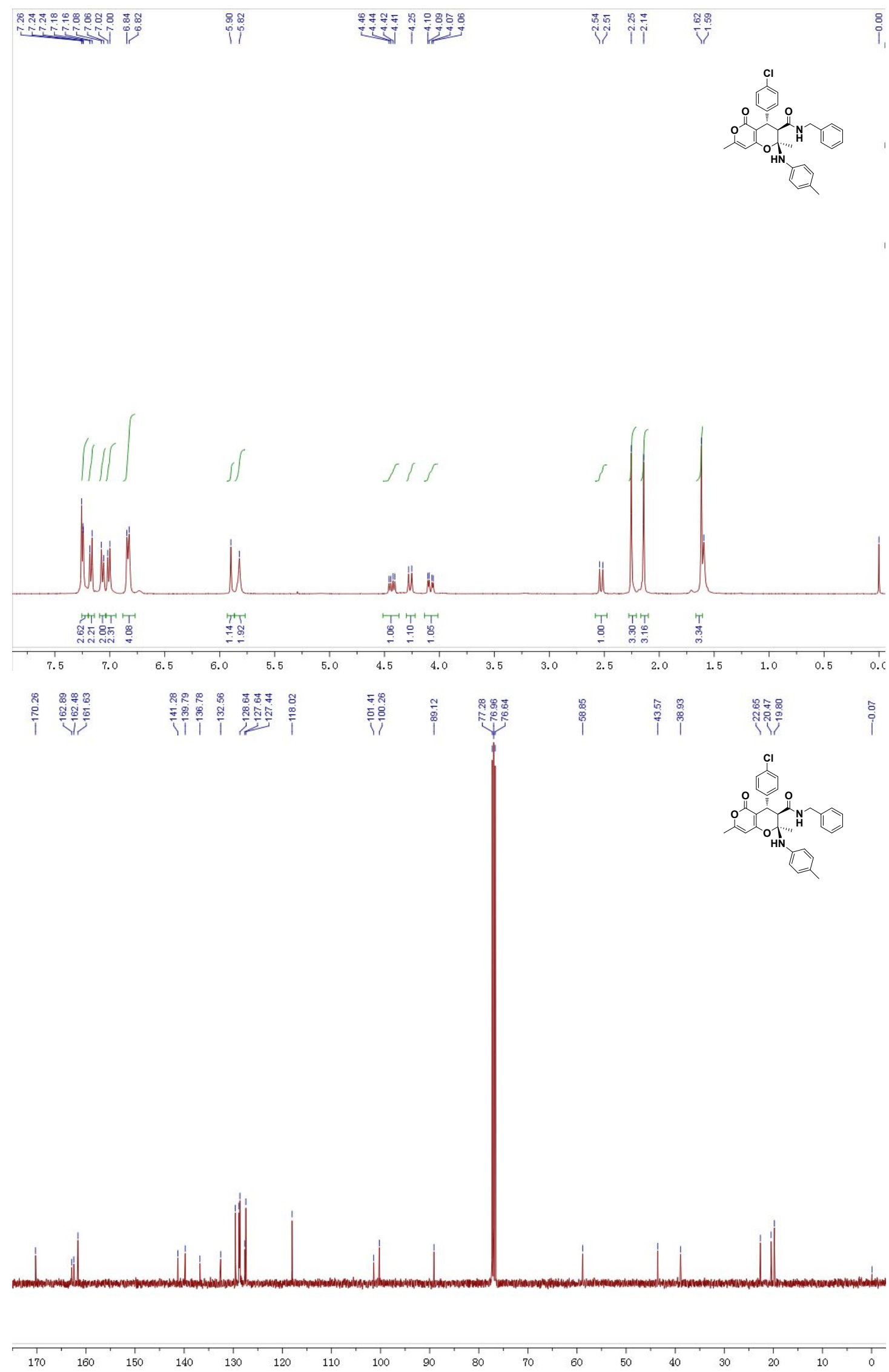


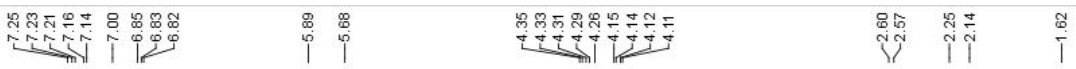
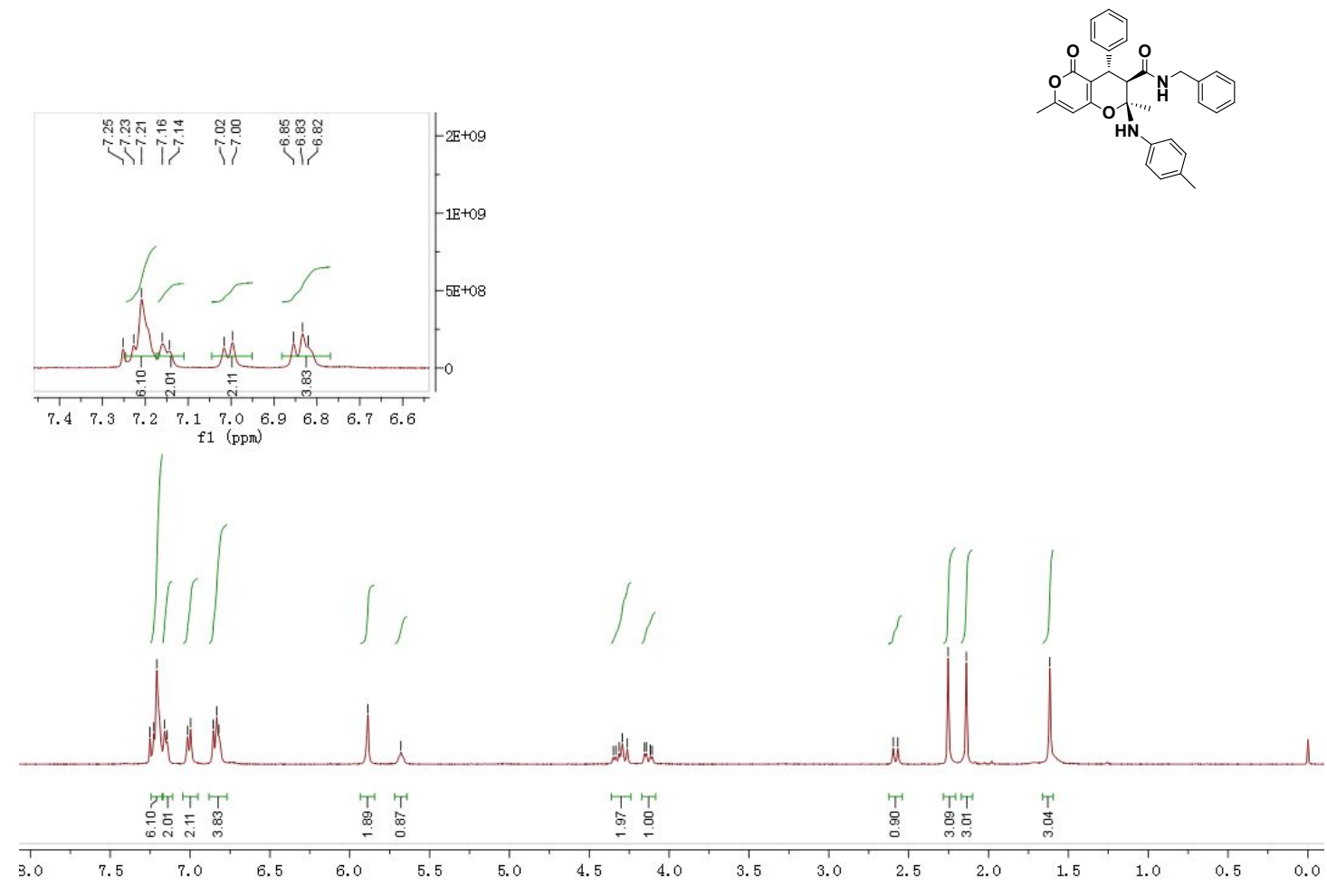

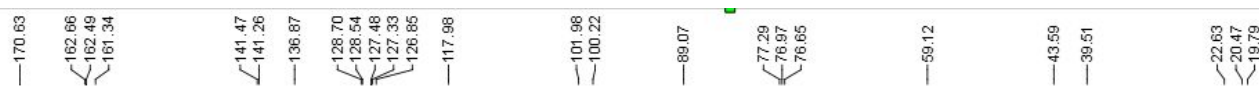
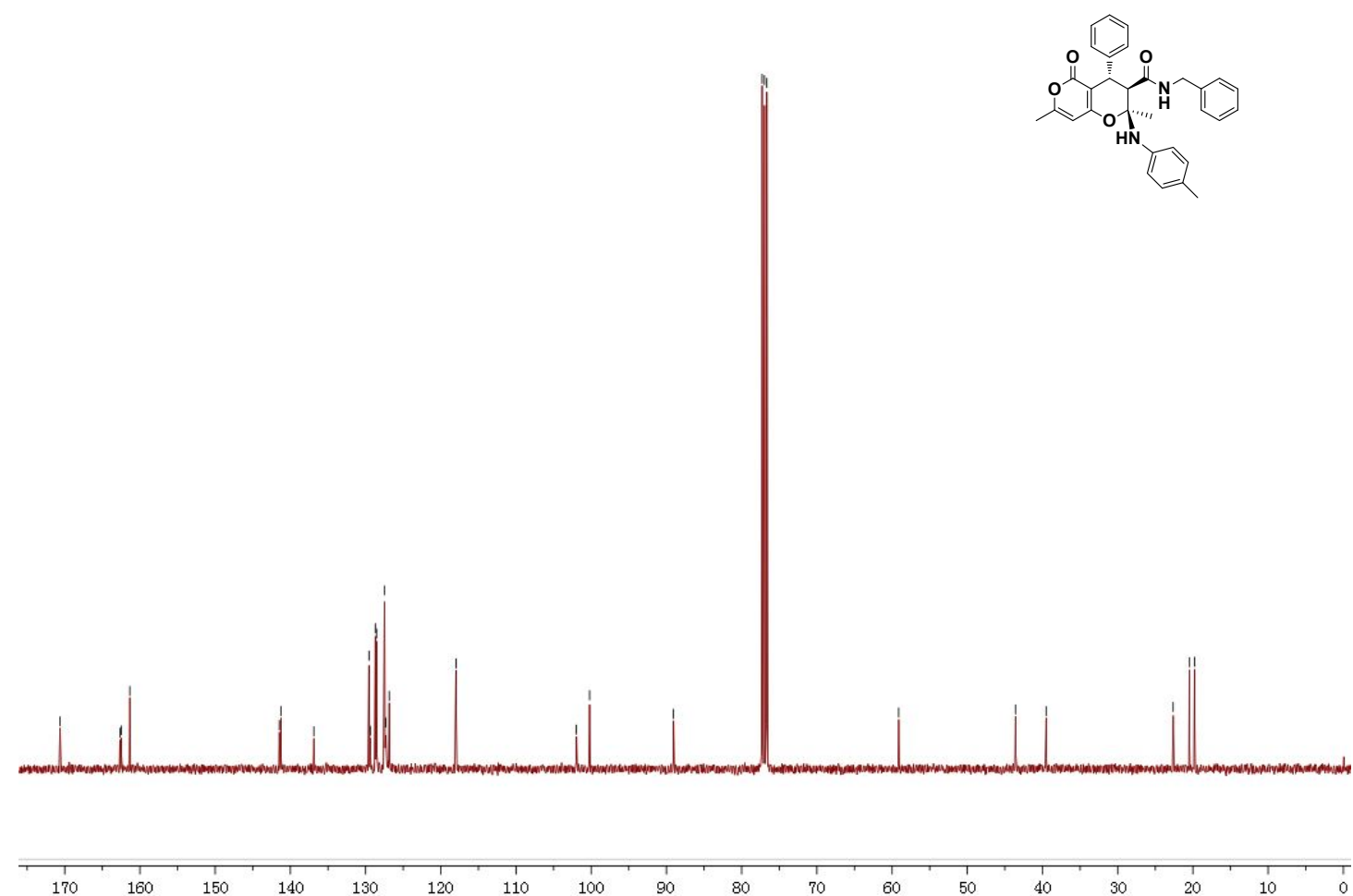


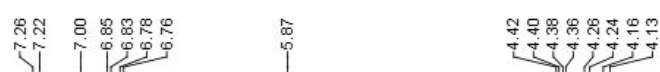

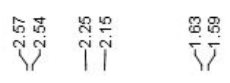

$\stackrel{8}{i}$
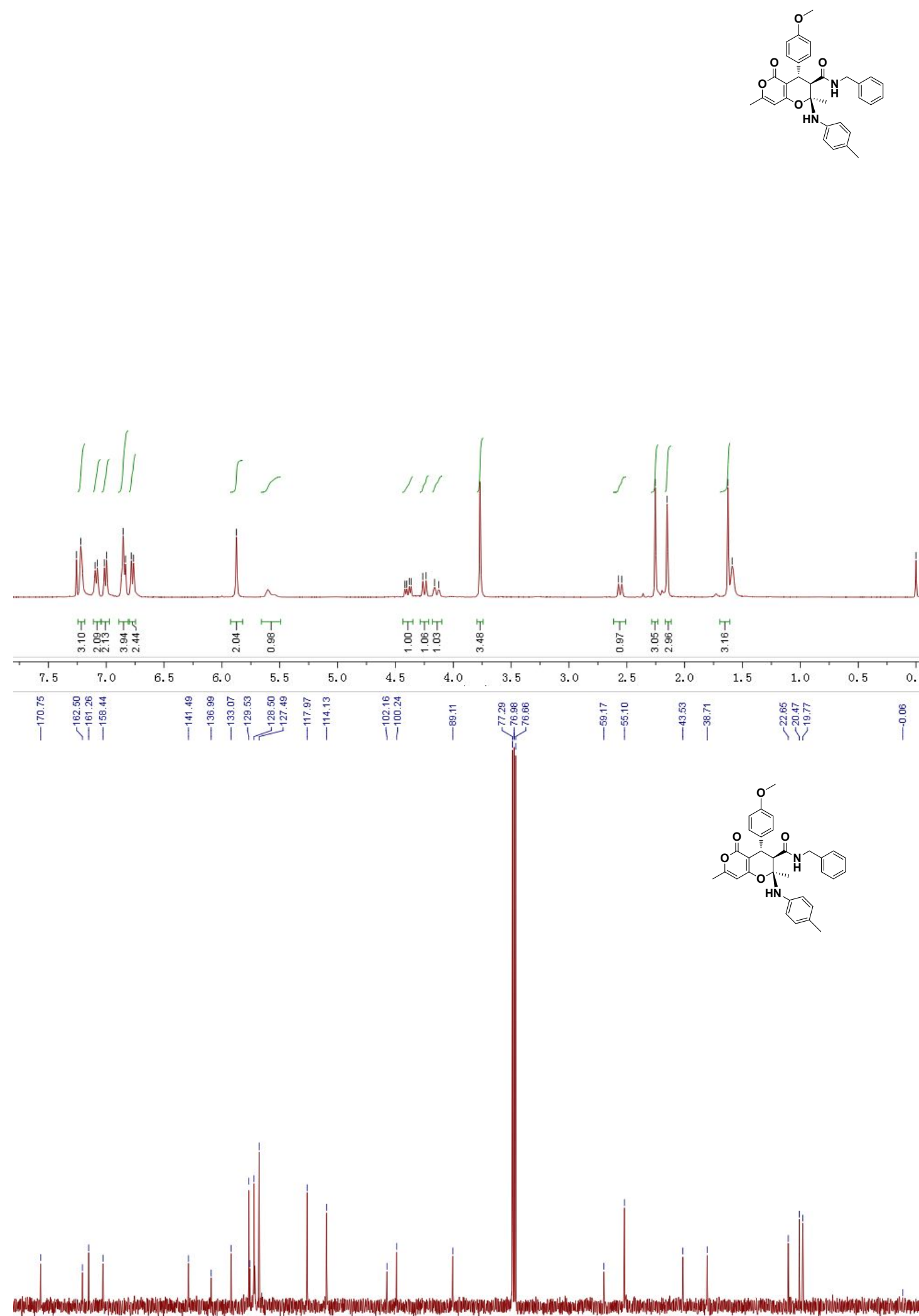

$\begin{array}{lllllllllllllllll}1 & 170 & 160 & 150 & 140 & 130 & 120 & 110 & 100 & 90 & 80 & 70 & 60 & 50 & 40 & 30 & 1 \\ 1 & 10 & 1\end{array}$ 

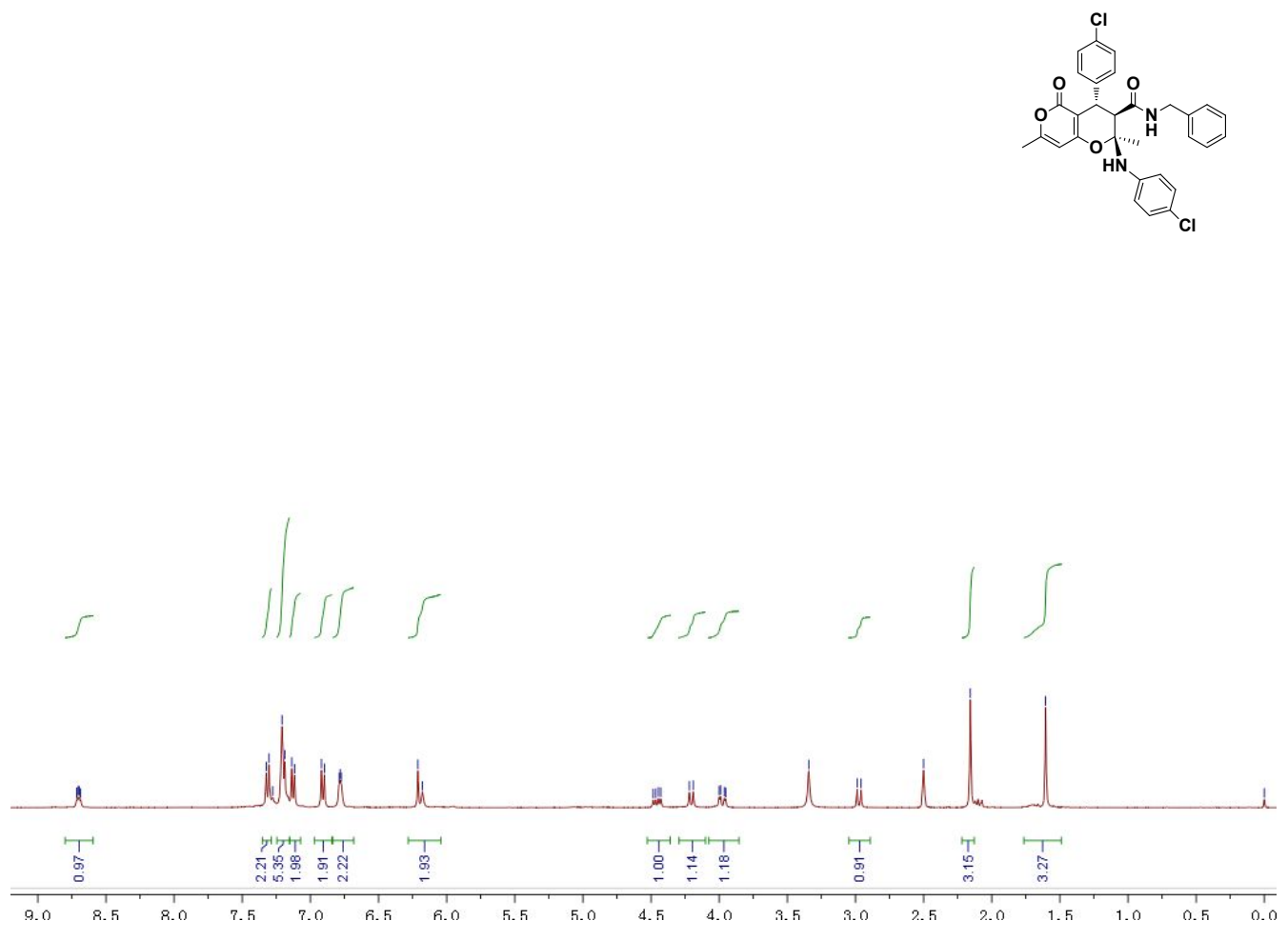

함ำ

$\infty$
$\infty$
$\infty$
$\infty$

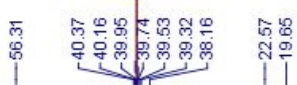

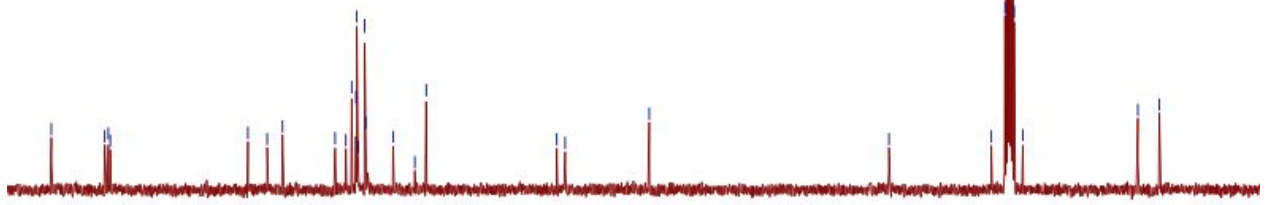

(1)

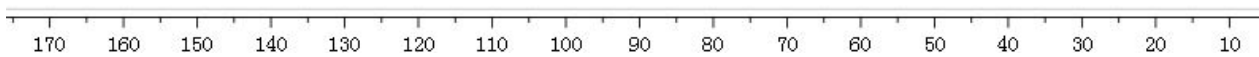




\section{Copies of LC-MS spectra with a chiral column for $6\{1,1,3,1\}$ :}
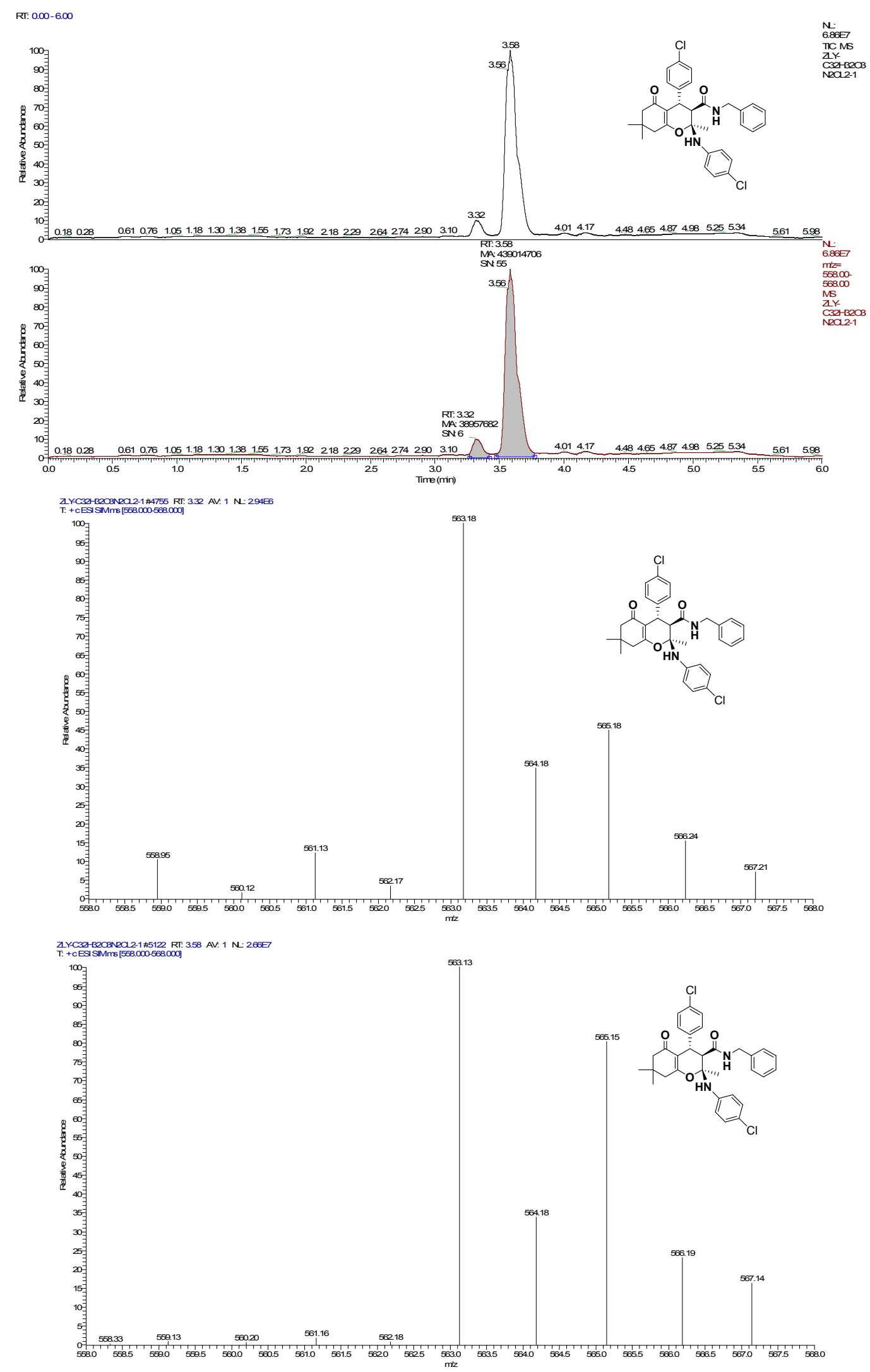